Florida International University FIU Digital Commons

11-18-1997

\title{
Social and cultural influences in the formation of identity: a cross-national/cultural study.
}

Ervin Briones

Florida International University

DOI: $10.25148 /$ etd.FI14051842

Follow this and additional works at: https://digitalcommons.fiu.edu/etd

Part of the Psychology Commons

\section{Recommended Citation}

Briones, Ervin, "Social and cultural influences in the formation of identity: a cross-national/cultural study." (1997). FIU Electronic Theses and Dissertations. 1886.

https://digitalcommons.fiu.edu/etd/1886

This work is brought to you for free and open access by the University Graduate School at FIU Digital Commons. It has been accepted for inclusion in FIU Electronic Theses and Dissertations by an authorized administrator of FIU Digital Commons. For more information, please contact dcc@fiu.edu. 


\title{
FLORIDA INTERNATIONAL UNIVERSITY \\ Miami, Florida
}

SOCIAL AND CULTURAL INFLUENCES IN THE FORMATION OF IDENTITY:

A CROSS-NATIONAL/CULTURAL STUDY

\begin{abstract}
A dissertation submitted in partial satisfaction of the
requirements for the degree of

DOCTOR OF PHILOSOPHY
\end{abstract}

IN

PSYCHOLOGY

by

Ervin Briones

1997 
To: Dean Arthur W. Herriott

College of Arts and Sciences

This dissertation, written by Ervin Briones, and entitled Social and Cultural Influences in the Formation of Identity: A Cross-National/Cultural Study, having been approved in respect to style and intellectual content, is referred to you for judgement.

We have read this dissertation and recommend that it be approved.

Wendy \&ilverman

Patricia Telles-Irvin

Jonathan Tubman

William Kurtines, Major Professor

Date of Defense: November 18, 1997

The dissertation of Ervin Briones is approved.

Dean Arthur W. Herriott

College of Arts and Sciences

Dr. Richárd L. Campbell

Dean of Graduate Studies

Florida International University, 1997 
I dedicate this dissertation to my wife Lazara and my children Janine Felisha, Ervin Josué, and Jocelyn Gabriela. 


\section{ACKNOWLEDGEMENTS}

First, I wish to acknowledge and thank my God, the main source of my motivation.

In addition, I wish to thank my major professor, Dr. Kurtines, for his insight, ideas, knowledge, support, guidance and example.

My parents and siblings also deserve recognition. They share my failures and successes.

My heartfelt appreciation to Xiaohui Guo and collaborators in China, Dr. Clary Milnistky-Sapiro of the Universidade Federal Do Rio Grande Do Sul in Portoalegre, Brazil, Dr. Iordanis Kavathatzopoulos of Upssala Universitet in Sweden, Dr. Liliana Wolf of Universidad de San José, Costa Rica, and Dr. Gustavo Carlo of the University of Nebraska at Lincoln for their invaluable help in collecting the data and/or translating the used instruments.

Finally, a special thanks to the members of my committee for their beneficial comments and criticisms.

Without any of the ones I have mentioned, this dissertation would not have been accomplished. 


\section{ABSTRACT OF THE DISSERTATION \\ SOCIAL AND CULTURAL INFLUENCES IN THE FORMATION OF IDENTITY:}

A CROSS-NATIONAL/CULTURAL STUDY

by

\section{Ervin Briones}

\section{Florida International University, 1997}

\section{Professor William M. Kurtines, Major Professor}

This multi-site, multi-ethnic/cultural study examined the effects of variation between ethnic/cultural groups and the effects of institutional variation within ethnic/cultural groups on identity formation. The participants were 892 late adolescent college students from six sites in 5 countries (Brazil, China, Costa Rica, US, and Sweden) representing different linguistic and ethnic/cultural traditions living in the context of varied social conditions. As hypothesized, there were significant differences in the proportion of identity statuses between sites in the Personal domain, $x^{2}(20$, $N=858)=164.78, \underline{p}<.001$, the Interpersonal domain, $X^{2}(20, N=858)=145.69, \underline{p}$ $<.001$, and the World View domain, $X^{2}(20, N=858)=120.89, \underline{p}<.001$, but the distribution of the differences was more complex than expected. In addition, there were significant differences in Identity Satisfaction among sites, $F(15,2325)=12.65$, $\underline{p}<.001$. Further univariate analyses revealed that differences among sites were found on Identity Satisfaction in the personal, interpersonal and world view domain. The direction of the differences, however, were more complex than hypothesized. 
The second hypothesis was confirmed but only with the world view identity status and not for each of the six sites. Stepwise discriminant analyses showed that Identity status in the world view domain was predicted by Institutional Support in Nebraska, gender and Institutional Change in Brazil, and Institutional Access in China. Lastly and as hypothesized, some Institutional Attributes significantly predicted Overall Identity Satisfaction in all sites as revealed by multivariate regression analyses, except in Sweden, $F(5,79)=.660, p=.65$. These findings extend the literature on identity formation not only by having investigated how culture influences the process of identity formation with samples representing different ethnic/cultural and linguistically different populations but also by empirically testing the role that social processes play in identity formation at the cross-cultural level. 
Introduction

Marcia's Framework for Operationalizing Identity Formation . . . . . . 3

Processes That Influence Identity Formation $\ldots \ldots \ldots \ldots \ldots$

The Effects of Ethnic/Cultural Variation on Identity Formation $\ldots \ldots .8$

The Effects of Social Institutions on Identity Formation . . . . . . . 16

A Co-constructivist Framework for Conceptualizing and

Operationalizing Identity Formation $\ldots \ldots \ldots \ldots \ldots 24$

The Formation of an Identity $\ldots \ldots \ldots \ldots \ldots \ldots \ldots$

Transcultural and Culture Specific Dimensions of Identity . . . 29

Goals of the Proposed Study $\ldots \ldots \ldots \ldots \ldots \ldots \ldots \ldots$

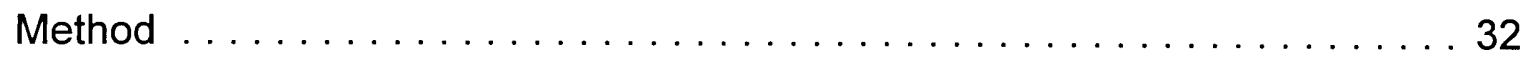

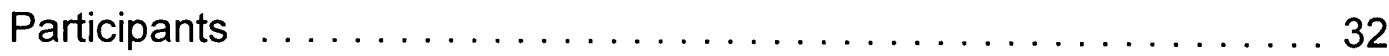

Measures .......................... 36

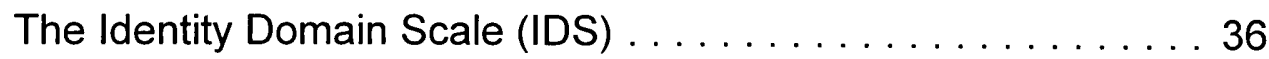

Scoring the IDS $\ldots \ldots \ldots \ldots \ldots \ldots \ldots \ldots \ldots$

The Social Institutions Rating (SIR) $\ldots \ldots \ldots \ldots \ldots \ldots$. . . 40

Scoring the $\operatorname{SIR} \ldots \ldots \ldots \ldots \ldots \ldots \ldots \ldots \ldots$ 


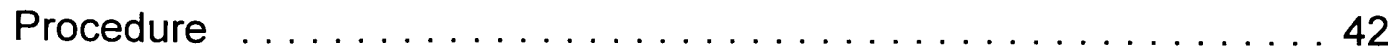

Results ................................ 44

Psychometric Analyses . . . . . . . . . . . . . . . . . . . . . . . 44

Factor Analysis . . . . . . . . . . . . . . . . . . . 44

Item Analysis $\ldots \ldots \ldots \ldots \ldots \ldots \ldots \ldots \ldots \ldots \ldots \ldots \ldots \ldots \ldots \ldots$

Internal Consistency Reliability $\ldots \ldots \ldots \ldots \ldots \ldots \ldots$

Hypotheses Testing . . . . . . . . . . . . . . . . . 47

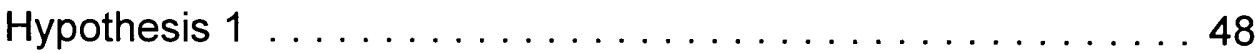

Personal Development Status .............. 49

Interpersonal Development Status . . . . . . . . . 50

World View Status . . . . . . . . . . . . . . . . 51

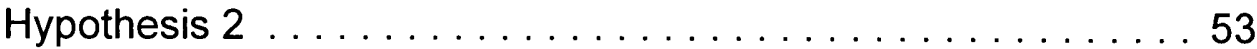

Hypothesis $3 \ldots \ldots \ldots \ldots \ldots \ldots \ldots \ldots \ldots$

Nebraska . . . . . . . . . . . . . . . . . . 59

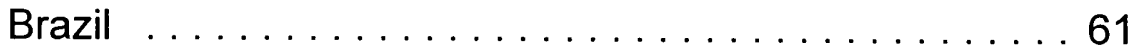

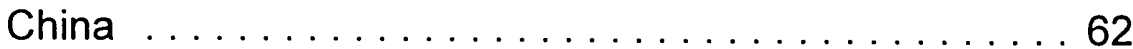

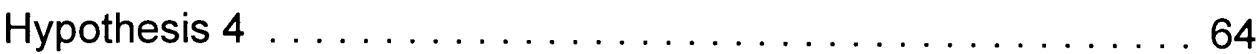

Discussion and Summary $\ldots \ldots \ldots \ldots \ldots \ldots \ldots \ldots \ldots \ldots \ldots \ldots \ldots$

Research Question $\ldots \ldots \ldots \ldots \ldots \ldots \ldots \ldots \ldots \ldots \ldots \ldots \ldots$ 
Summary of Results $\ldots \ldots \ldots \ldots \ldots \ldots \ldots \ldots \ldots \ldots \ldots \ldots \ldots \ldots$

Integration of Results with Existing Literature $\ldots \ldots \ldots \ldots . \ldots 69$

Interpretation of Results $\ldots \ldots \ldots \ldots \ldots \ldots \ldots \ldots \ldots \ldots \ldots$

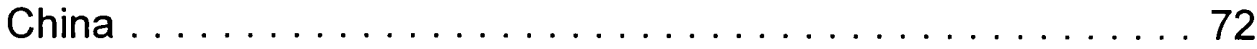

Sweden ........................ 76

Commonality of Results Between China and Sweden . . . . . 78

Brazil and Costa Rica . . . . . . . . . . . . . . . 80

Miami and Nebraska $\ldots \ldots \ldots \ldots \ldots \ldots \ldots \ldots$

Transcultural versus Culture Specific Sociocultural Factors . . . . 87

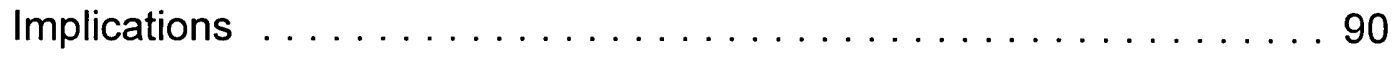

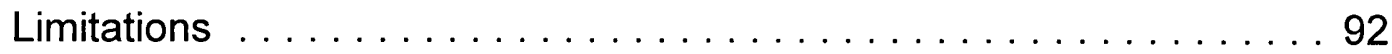

Suggestions for Future Research $\ldots \ldots \ldots \ldots \ldots \ldots$

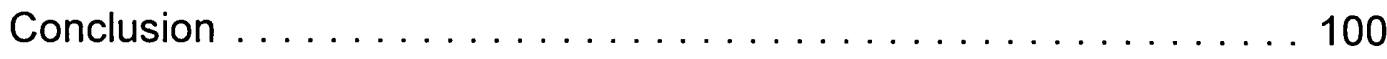

References . . . . . . . . . . . . . . . . . . . . . . . . . . . . . . . . 102

Appendices . . . . . . . . . . . . . . . . . . . . . . . . . . . 120 


\section{LIST OF TABLES}

Page

1. Gender Distribution, Expected Values, and Adjusted Standardized

Residuals by Site

2. Grade Distribution, Expected Values, and Adjusted Standardized

Residuals by Site . . . . . . . . . . . . . . . . . . . . . . . . 191

3. Marital Status Distribution, Expected Values, and Adjusted Standardized

Residuals by Site

4. Reliability Coefficients for the Total Identity Satisfaction, Personal,

Interpersonal, and World View Satisfaction Scales by Site 193

5. Factor Structure Matrix of the Attribution and Evaluation Ratings

Across Social Institutions in Miami 194

6. Factor Structure Matrix of the Attribution and Evaluation Ratings Across

Social Institutions in Nebraska .

7. Factor Structure Matrix of the Attribution and Evaluation Ratings Across

Social Institutions in Brazil 196

8. Factor Structure Matrix of the Attribution and Evaluation Ratings Across

Social Institutions in Costa Rica

9. Factor Structure Matrix of the Attribution and Evaluation Ratings Across

Social Institutions in China 198 
10. Factor Structure Matrix of the Attribution and Evaluation Ratings Across

Social Institutions in Sweden

11. Reliability Coefficients for the Overall Institutional Attributes Scale and the Overall Institutional Evaluation Scale . . . . . . . . . . 200

12. Reliability Coefficients for the Institutional Attributes Scales of Social

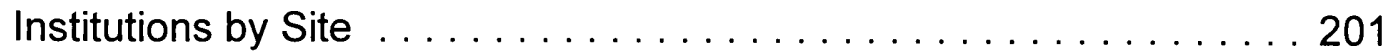

13. Reliability Coefficients for the Institutional Evaluation Scales of Social Institutions by Site . . . . . . . . . . . . . . . . . . . . . . . 202

14. Personal Development Status Distribution, Expected Value, and Adjusted Standardized Residuals by Site 203

15. Interpersonal Development Status Distribution, Expected Value, and Adjusted Standardized Residuals by Site . . . . . . . . . . . . . . 204

16. World View Status Distribution, Expected Value, and Adjusted

Standardized Residuals by Site . . . . . . . . . . . . . . . 205

17. Mean of Satisfaction/Distress Rating for the three Identity Domains and Standard Deviation by Site . . . . . . . . . . . . . . . 206

18. Discriminant Analyses Predicting Identity Statuses in the World View Domain in Nebraska

19. Discriminant Analyses Predicting Identity Statuses in the World View Domain in Brazil . 208 
20. Within Groups Correlations and Discriminant Function Coefficients

Predicting Identity Statuses in the World View Domain in Brazil . . . . 209

21. Results of Discriminant Analyses Predicting Identity Statuses in the World View Domain in China . . . . . . . . . . . . . . . . . . . . . 210

22. Association of Institutional Attributes and Total Identity Satisfaction

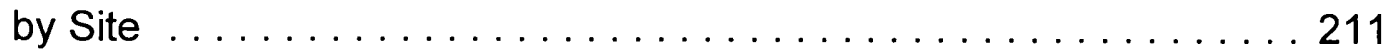


One of the most widely researched stages in Erikson's $(1963,1968)$ developmental theory is ego identity. In the book "Identity: Youth and Crisis", Erikson (1968) provides rich and intricate descriptive statements about the identity construct that encompasses biological, social, and psychological components. The construct of identity is used by Erikson to describe the adolescence stage in the life cycle that confronts the individual with a complex and difficult challenge (and responsibility), namely, that of choosing and fulfilling the goals and values that give the individual's life direction and purpose. For Erikson, ego identity is characterized by an awareness that one possesses a coherent representation of the self based on previous and present experiences that provide the individual with reference or guidance for future plans. This coherent representation of the self is formed in the context of social exchanges in which the individual not only confirms and validates him or herself but also is recognized and validated by society which in turn provides the individual with a sense of sameness and continuity over time (a sense of identity).

The process of acquiring an identity -that of choosing and fulfilling the goals, roles and values that provide the individual with a sense of purpose and meaning in life- is described as a product of the interactional co-development of individual needs and societal demands that takes place both at a conscious and unconscious level in the individual (Marcia, 1992). Particularly during adolescence the individual is confronted with an array of self perceptions and 
available roles from which he or she has to choose and also integrate in a consistent and cohesive manner. The potential for integrating the individual's self perceptions and available roles is conceptualized occurring in part as result of the matching between the individual's capabilities, interests, values and life history on the one hand, and society's demands, resources, and rewards on the other. Erikson (1968) thus, assigns the location of this process taking place not exclusively at the individual level as if occurring in a social vacuum but as he asserted "is also in the core of his communal culture" (p. 22).

As modern society has become increasingly more complex, diverse and pluralistic, the array of self perceptions and available roles that define the individual's identity (and living up to the responsibility for these choices) has become increasingly more difficult and complex given that the youth have to "synthesize" often incompatible models, identifications, and ideals offered by his/her society. The more incompatible the components from which the individual has to choose from, the more uncertain and difficult is the process of integrating these experiences into a coherent unity. The process of integrating life experiences into a coherent unity might lead the individual into a "crisis" or period of undefined identity. Crisis refers to a period of uncertainty about one self, one's goals, roles, and values that often interferes with adaptive living in one's environment. Indeed, for many adolescents this period of uncertainty may even produce clinically significant psychological distress (Niemi, 1988) whereas others in an attempt to find an identity, adopt maladaptive identifications in the 
form of socially deviant peer groups (e.g., joining street gangs, extremist groups). If a decision is made by the adolescent with regard to pressing issues such as the selection of an occupation or career, gender role, friendship and group membership, moral issues, religion, etc., an assured sense of self (personal identity) is accomplished. Identity confusion or diffusion results if there is not an active search for the resolution of these issues or if the adolescent is not able to consolidate in a cohesive and consistent manner the different options.

Marcia's Framework for Operationalizing Identity Formation

Several authors have attempted to operationalize Erikson's concepts of ego identity and identity crisis (e.g., Baker, 1971; Bronson, 1959; Bunt, 1962; Byrd, 1971; Marcia, 1966; Murray, 1964; Rasmussen, 1964; Simmons, 1970). However, Marcia's (1964) operationalization of this comprehensive and rather elusive construct has been the one most often used by researchers. Marcia (1966) developed a semistructured interview that assesses personal identity in relation to several domains of concern to the individual and the process by which identity develops. This classificatory system uses four statuses representing a continuum from a less advanced sense of ego identity to a more advanced sense of ego identity. The four statuses are: (a) identity diffusion, (b) foreclosure, (c) moratorium, and (d) identity achievement.

The identity diffusion status is characterized by individuals who are not committed to any particular role and are not actively searching for one either. In other words, these adolescents are not engaged in the decision-making process 
produced by the availability of the different options provided by society mainly in the areas of occupation and ideology and thus, may or may not experience a crisis.

The identity foreclosure status is identified in individuals who lacked an identity crisis and thus did not explore available options but are nevertheless committed. Generally, these commitments represent those values and roles suggested by parental figures and are assumed without being questioned or examined and are therefore parental rather than self-determined.

The moratorium status is considered to precede identity achievement. The individuals classified in this status are experiencing a crisis due to their active exploration or consideration of the different options available in society but have not been able to choose from the available alternatives or commit themselves.

The achievement status is represented by those who have moved beyond the moratorium status and have been able to choose the goals, roles, and values that give their life direction and purpose. The achievement status then is represented by individuals who are convinced of what they want to do with their lives and are pursuing a course of action towards their commitments.

Commitment involves having a strong conviction that a decision has been made in areas of personal interest as well as engaging in appropriate implementing activities. 
it needs to be clarified that even though Marcia's classificatory system assigns people a particular identity status, it does not mean that these identity statuses represent a permanent state with no possibilities of identity status change. In fact, a longitudinal study (Waterman \& Waterman, 1971) as well as cross sectional studies have found progressional changes in identity formation with advancing age. For instance, Christopherson, Jones, and Sales (1988) found that early adolescents fall mostly into the diffusion and foreclosure categories in contrast to late adolescents who were more often classified in the achieved status. Other studies have also found similar results with college students (Benson, Harris, \& Rogers, 1992; Prager, 1986). These studies illustrate a type of sequential change in identity formation that have been labeled by Waterman (1982) as identity "progressions" which are changes from nonexplored statuses (i.e., diffusion and foreclosure) to explored statuses (i.e., moratorium and achieved). Identity formation, however, can also be described as "regressive" when the changes in identity formation occurr from explored to non-explored statuses (see e.g., Krogger, 1996). In other words, there can be fluctuations or shifts in identity statuses throughout time ( Adams \& Fitch, 1982; Waterman, Geary, \& Waterman, 1974; Waterman \& Goldman, 1976).

The operationalization of the identity construct in terms of these four statuses has linked identity status during adolescence to personality correlates (Muss, 1975; Adams, Abraham, \& Markstrom, 1987; Adams, Ryan, Hoffman, Dobson, \& Nielsen, 1985; Coté \& Levine, 1983; Clancy \& Dollinger, 1993; Dellas 
\& Jernigan, 1987, 1990; Donovan, 1975; Tesch \& Cameron, 1987), other psychological developmental processes (Abraham, 1983; Adams, Dyk, \&

Bennion, 1990; Adams \& Shea, 1979; Wallace, Serafica, \& Osipow, 1994) and many other psychological and behavioral indicators (Waterman, 1982) as well as environmental factors (see Waterman, 1985, for a review). Marcia's conceptualization of ego identity, nonetheless, has been criticized by others (Coté \& Levine, 1988a, 1988b) who argued that this account of identity is not only short sighted given that it does not contain the fullness of the description that his original author intended, but also that the paradigm itself is incongruent with Erikson's theoretical assumptions. On the other hand, other investigators (Waterman, 1988) justify the paradigm and present ample evidence that warrant its use, particularly when the categories derived from such an operationalization are not viewed as a global measure of identity. In addition, investigators who advance the identity status paradigm should be aware that this paradigm taps into that process which takes place more at the conscious level and that there are other processes that influence identity formation not being captured by it. Processes That Influence Identity Formation

Although, according to Erikson many processes influence identity formation (e.g., anatomical, psychological, social, historical), the process that has received by far the largest amount of attention in the identity literature is a psychological process called exploration. The process of exploration is an active one that involves questioning of the attitudes, beliefs, roles, and values that have 
been handed out by the family, community, society and ultimately the culture in which one is embedded. Some or all of these attitudes, beliefs, roles, and values are retained, others are replaced while still others are further modified depending on the kind of resolution the individual arrives when making his or her commitments.

During exploration, the individual not only searches among already available alternatives offered in his or her society (in the forms of roles and ideologies) but also formulates actual and potentially new alternatives. Thus, the process of identity formation has to include a certain degree of involvement from the part of the individual. This involvement can be experienced at the cognitive and emotional level at what it could be considered as a purely psychological exploration. For other individuals, however, the process of identity formation might also include a period of actual behavioral venture (with cognitive and emotional components as well) that culminates with a commitment to particular areas of interest and the preference for particular roles. As previously noted, this process may produce considerable psychological distress for many individuals and may involve a considerable period of time of uncertainty and conflict.

The research conducted on exploration has contributed to our understanding of the formation of an identity. However, although most of the research on identity formation has been conducted within a psychosocial developmental framework that explicitly recognizes the role of social processes, little research has been conducted that systematically investigates how social 
processes influence the formation of an identity. Indeed, one of the arguments that challenges Marcia's operationalization of identity is that it does not take into account the interplay of psychological and socio-cultural factors (Coté \& Levine, 1988a,b, 1989, 1992), an interplay that is emphasized in all Erikson's writings dealing with identity issues. The study of the interplay of psychological and socio-cultural factors thus, is in need of empirical investigation.

The research to be conducted as part of this dissertation sought to extend the literature on identity not only by investigating how culture influences the process of identity formation with cross-national/cultural samples but also by investigating cross-culturally the role that social processes play in identity formation. Social processes that influence identity formation are investigated at two distinct levels. At one level, the study examines the effects of ethnic/cultural variation on the process of identity formation between ethnic/cultural groups. At a second level the study investigates the effects of social institutions on identity formation within ethnic/cultural groups.

\section{The Effects of Ethnic/Cultural Variation on Identity Formation}

Before reviewing the literature on the effects of ethnic/cultural variation on identity, a brief definition of culture and ethnicity is in order. Even though culture has been recognized as one of the most important factors influencing human behavior in general (Nugent, Lester, \& Brazelton, 1989) and identity development in particular (Erikson,1963, 1968), culture itself has been difficult to be defined in part because of the complexity to capture in a single definition the 
variety of aspects that this concept encompasses. Culture, however, has been generally described as a social mechanism influencing all aspects of human behavior including norms (conventions, mores, laws), language, social institutions and values together with a society's technology and its material products (Hunt \& Collander, 1987, p. 116). A closely related concept that social and natural scientists have attempted to define is ethnicity. Even though according to McDavid and Harari (1974), Social Psychologists, Biologists, and Physical Anthropologists do not even agree in the definition of ethnicity, it has been described in terms of several dimensions including race, language, religion, mores, customs, traditions and values (DeFleur, M., D'Antonio, \& DeFleur, 1981) among other things. In the identity literature, not surprisingly, ethnicity has been operationalized inconsistently. Most of the studies in the psychological literature, however, have operationalized ethnicity in terms of self-identification with a particular cultural group. Participants chose their ethnicity from a provided list or choose a self-generated ethnic identifier. This study also used self-identification to index ethnicity which has been the method most commonly employed in the identity literature that has used Marcia's identity status paradigm to study identity development.

There is a large and growing research literature based on Marcia's (1966) paradigm (e.g., Adams, Bennion, \& Hugh, 1987; Adams, Shea, \& Fitch, 1979; Grotevant \& Adams, 1984; Marcia, 1966; Marcia 1980; Marcia, Waterman, Matteson, Archer, \& Orlofsky, 1993; Waterman, 1982, 1985, 1992). Most of this 
work, however, has been conducted with populations mainly represented of the mainstream cultural tradition in the United States. Recently, there has been a growing interest in investigating the impact of diverse ethnic and cultural traditions on identity formation in the United States particularly among Blacks and Hispanics but also among Native and Asian Americans (Abraham, 1983, 1986; Aries \& Moorehead, 1989; Bacho, 1994; Briones, 1995; MarkstromAdams, Ascione, Braegger, \& Adams, 1987; Rotheran-Borus, 1989; Streitmatter, 1988; Telles, 1983; Watson \& Protinsky, 1991).

Some studies on ego-identity status have found that ethnic minorities tend to be more foreclosed than nonminority samples (e.g., Abraham, 1986; Hauser, 1972; Streimatter, 1988). Other studies, however, have not found ethnic minorities being more foreclosed or experiencing less moratorium than nonminority groups (e.g., Abraham, 1983; Briones, 1995; Markstrom-Adams, Ascione, Braegger, \& Adams, 1987; Telles, 1983). Briones (1995) has suggested that these finding inconsistencies might be partly explained by the differences within minority subgroups employed in the literature which are taken from different geographic regions in the U.S. For instance, some Hispanic subgroups have different socio-demographic and cultural/historical characteristics that may contribute to different identity outcomes in somewhat varied social, economic, and political contexts within the varied Hispanic communities in the U.S. In addition, some historical changes have to be taken into consideration when comparing results across time and across ethnic groups. 
African Americans, for example, are now more engaged in the identity searching process than they were twenty years ago (see e.g., Aries \& Moorehead, 1989; Watson \& Protinsky, 1991 compared to Hauser, 1972). There has been growing interest in ethnic identity formation among minority groups from an Eriksonian perspective using Marcia's (1966) identity status paradigm (e.g., Phinney, 1993, 1992, 1989; Phinney \& Alipuria, 1990; Phinney \& Chavira, 1992; Rotheram-Borus, 1989). On studies of ethnic identity formation, minorities in the U.S. have shown similar (Phinney \& Alipuria, 1990) if not higher ethnic identity scores than white youth (Phinney, 1993, 1992, 1989).

There is also a small but growing literature that has investigated identity status in diverse ethnic and national/cultural traditions worldwide. An early effort was made by Chapman and Nicholls (1976) to compare the occupational identity status (foreclosed, diffused, moratorium, and achieved) of two ethnic groups in New Zealand, the Maoris (polynesians) and Pakehas (whites). More Polynesians were categorized as identity diffused and more whites as identity achieved.

This early effort was followed by Matteson (1977) who studied ego-identity development with a sample of young adolescents in Denmark in terms of the degree of exploration and commitment in four areas: occupation, values, politics, and sex roles. This study showed that specific identity content areas (sex roles and the search for values in particular) were the strongest predictors of ten personality variables. Similarly, Ochse and Plug (1986) compared South African 
white and black men and women in an effort to validate Erikson's theory of personality development including the identity stage. As revealed in other studies, in general, black participants showed a less coherent sense of identity than did white participants. In addition, black participants appeared to integrate less successfully the personality components resulting from earlier crises.

More recently, Botcheva and Zlatkov (1994) presented the results of an extensive longitudinal study of identity issues taking into consideration the historical context in which adolescents live and experience the process of identity formation. In Botcheva and Zlatkov's study, the historical context refers to the transitional period experienced in Bulgaria from a totalitarian system (1989) to the establishment of democratic institutions (1991) and marketization in 1992. Bulgarian youth reported an increased interest and broadened values in economic and cultural activities but a diminished interest and activity in political matters during the period of establishment of democratic institutions (1992) in marked contrast to the interest in political aspects during the totalitarian regime in 1989. While during the transition from totalitarianism to democracy youth became more active and sensitive in some areas, they defined themselves as having less influence over the social environment. A noteworthy finding was that the youth from Bulgaria felt worse about their social environment which may reflect the short-term effects of drastic social, economic, and political changes.

Kroger (1993), using a time-lag design, also reported the identity status in occupational, political, religious and sex role values of a group of college 
students within the context of a changing partly socialized economy (1984) that moved to a free market economy (1990) in New Zealand. The identity status distributions were significantly different between males and females for the identity areas (i.e., occupation, political and religious views, and sex role values) in 1990 as compared to non-significant differences between males and females assessed in 1984. Females showed a significant decrease in achievement and increase in foreclosure ratings. Specific identity areas (religious and political values) changed their degree of importance for these college students. However, these changes were observed among females only. Similar to Botcheva and Zlatkov's (1994) results, politics was rated least important by the 1990 sample replacing religion as the least important domain for late adolescents in 1984 .

In spite of this growing interest in studying identity in different countries worldwide there has been to this writer's knowledge only two published studies that have used cross national/cultural samples. McClain (1975) studied teenagers from four european cities (Brussels, Munich, Charleville-Mezieres, and Malaga) and white and black samples from Knoxville, Tennessee. Results indicated consistent differences among these groups on six scales that assessed resolution regarding the first six Eriksonian psychosocial developmental tasks. Participants from Brussels, Munich, and Knoxville (white) showed higher mean scores than those from Knoxville (Black), Charleville-Mezieres (France), and Malaga (Spain). These last three communities with lower scores have lower 
standards of living compared to the other communities which according to the authors may provide their youth with fewer economic resources to facilitate personal growth. Unfortunately, this study addressed the first six personality stages theorized by Erikson and did not focus either on the process of identity formation nor on cultural variations along the dimensions or components of identity.

In a study of Turkish and American college students, Taylor and Oskay (1995) found that American adolescents scored higher on identity achievement and moratorium status and higher on foreclosure status than their Turkish counterparts in the interpersonal and ideological content domains of Bennion and Adams' (1986) Extended Objective Measure of Ego Identity Status (EOMEIS). An unexpected finding among American respondents, however, was the higher foreclosure scores in the ideological domain of the EOM-EIS compared to Turkish respondents. Also, Turkish adolescents reported more authoritarianism by their parents in managing the family than did the American adolescents. Nonetheless, the two groups did not differ in their levels of self-esteem although for both groups self-esteem was positively correlated with identity achievement in the ideological and interpersonal domains of the EOM-EIS. According to the authors, the lower levels of identity achievement for Turkish students may be a manifestation of the more controlling nature of Turkish families that direct and organize the adolescents' lives in a thorough but culturally acceptable manner (Taylor \& Oskay, 1995). It has to be pointed out, however, that parental 
authoritarianism seems unlikely to be solely responsible for the lower level of identity achievement and moratorium among Turkish participants given that the scores of parental control had little influence on their children's identity formation. This was evidenced by the lack of significant correlations between authoritarian parenting or parent-adolescent conflict with most of the identity statuses in ideological and interpersonal domains (see Taylor \& Oskay, 1995, p. 16). Other social processes might be at work here.

While some studies illustrate indirectly the impact that social institutions can have on identity formation (e.g., Botcheva and Zlatkov, 1994; Kroger, 1994) studies that directly assess specific social institutions and how social institutions affect either identity formation or identity satisfaction have not been undertaken yet, with the exception of an exploratory study that compared Hispanic and Euroamerican college students (Briones, 1995). This author examined the relationship between satisfaction with social institutions and identity status among 103 college students. Identity statuses were assessed on three domains of identity development (personal development, interpersonal development, and world view). There were no significant effects of satisfaction with social institutions for the overall identity status (across domains) among Hispanics. Among Euroamerican participants, however, satisfaction with Economic Institutions was related to identity status, with Achieved participants scoring highest on Satisfaction with Economic Institutions and Foreclosed scoring lowest. There were no significant effects for Interpersonal Development Status 
nor for World View Status among Hispanics either. However, among Euroamericans there was a significant effect between Gender Roles and World View Domain Status with Undifferentiated participants (those without a distinguishable identity status) scoring highest on Satisfaction with gender roles and Foreclosed scoring lowest. These preliminary results seemed to provide tentative support for the theoretical relationship between social institutions and identity formation. However, the impact that social institutional change have on identity formation and life satisfaction under varied social, cultural, economic, and political contexts has not been studied directly. Nevertheless, there are some theoretical accounts that have provided an explanation of the possible effects that social institutions have on identity formation.

\section{The Effects of Social Institutions on Identity Formation}

The effects of social institutions on identity has always been recognized. Erikson (1968), for example, has described how institutions provide guidance and narrow choices for adolescents as they explore "ideologies" presented to them in the form of established social institutions (e.g., political, religious, and economic institutions). Furthermore, some institutions even allow for a specific time in which individuals are exposed to learn and experience specific roles (e.g. religious and educational institutions, the job market, etc.). While the effects of social institutions have been obviously recognized in the sociological literature (see e.g., Knight, 1992), the construct of social institution itself has been difficult to operationalize for sociologists. Indeed, the concept of social institution has 
been as difficult to operationalize as the psychological construct of identity. In fact, the study of social institutions and their definition is in and of itself an active field of scholarly endeavor within sociology (see e.g., Hallinan, Klein, and Glass, 1990).

Although not all sociologists agree on a definition of what a social institution is, there are common themes that have defined the field. Form (1990), for example, presents an overview of how four major sociological theorists have studied institutions and outline four main areas of institutional analysis: the substance or composition of institutions, their purpose or functions, the classification of institutions, and the types or range of social formations that institutions cover. Hunt and Colander (1987), on the other hand, define a social institution as "an established, complex pattern of behavior in which a number of persons participate in order to further important group interests" (p. 118). Social institutions can be seen then as being commonly organized around some central interest and/or need (Hunt \& Colander, 1987) that come to represent standardized customs, regulations, procedures, and solutions to specific goals as they reflect a particular set of beliefs and behavioral expectations (Herric, 1977). Examples of social institutions include: the family and kinship, marriage, social stratification, the government, economic, politicy, education, mass media, and religion among many others.

The effects of social institutions on identity formation are theorized to be broad and varied. This study, however, will focus on the effects on identity 
formation of one particular dimension of social institutions, namely, institutional change (Mayer, 1992). This study will focus on institutional change because of the important role it plays in identity formation. As social and cultural institutions change, this change may affect the individual's identity formation in many ways. Specifically, the social and cultural institutions in which individuals are embedded provide the context in which individuals search for, commit to, and fulfill their life goals and values. Consequently, when such institutions change, this change confronts the individual with new and sometimes difficult challenges that are the result of the restructuring of traditional roles and values as defined by these social institutions. For example, when gender roles as a social institution undergo a process of change, the ordinary normative challenge that the individual faces in taking on a gender identity that s/he feels comfortable with is exacerbated by the additional challenge of determining the personal appropriateness of newly emerging gender roles.

Furthermore, when the institutions that provide a social outlet for identity exploration and experimentation are experiencing significant changes themselves (e.g., changing social norms), they are more likely to provide the individual with less social clarity of what is acceptable and rewarded in a particular social and historical context. Thus, societies undergoing more intense institutional change -especially if it is chaotic in nature- are less likely to provide individuals with clear institutional practices that guide identity exploration. In addition, societies undergoing rapid change are less likely to offer to the 
individual institutionalized means that support the search process necessary for an appropriate identity exploration, commitment, and implementation. For example, an individual attempting to decide for an occupation or a suitable career major may find a stable society that provides varied job opportunities and career options as a conducive environment to experience with different career and occupational goals and roles (a psychosocial moratorium) which contribute to an eventual commitment to a course of action (i.e., an achieved identity). In contrast, a society with limited job opportunities or an unpredictable job market in the context of constantly changing legal and political reforms may exacerbate the problem confronted by the individual in identifying the options available in a particular historical period. Moreover, the individual may experience less than optimal social conditions (e.g., stability, availability of roles, clarity of institutionalized values) and unavailable institutional characteristics (e.g., institutional access, openness, support) necessary for the individual to experiment with the available alternatives. Finally, such a society may not be able to offer the individual with appropriate social means to support the commitment to a course of action which in turn can translate into a psychological sense of dissatisfaction with the quality of one's life.

Therefore, it is hypothesized that individuals in societies that are undergoing more extensive social institutional change with fewer institutional characteristics that support identity exploration will report lower levels of identity development and identity satisfaction than will individuals belonging to more 
stable societies with more supportive and accessible institutions. Institutional change, however, cannot be seen in and of itself as having an inherently negative connotation. Institutional change can be (and indeed is) positive as long as it furthers important group interests and is not so intense and chaotic as to deplete the individual's capacity to cope with such change. Institutional change can be thought of as having positive or negative impact on identity formation depending on whether these changes will foster or hinder individual and collective growth and development. Therefore, it is hypothesized that societies whose institutions are evaluated more positively by their members (whether they are changing or not) will have individuals with more advanced identity development and higher satisfaction with the quality of their lives than societies whose members evaluate their social institutions more negatively.

It appears that out of the five countries that were sampled in this study, Brazil, Costa Rica and China are societies experiencing more extensive social institutional change compared to the United States of America and Sweden. For instance, Brazil has experienced significant institutional transitions that have taken place in the context of change from a military government to a path of controlled political opening during the early seventies to more democratic forms of life in the eighties (Cavarozzi, 1992) that have extended to the present. These years have been markedly difficult for their fluctuating economy that witnessed for instance, the highest inflation in the nation's history in 1988 when consumer prices rose by more than 900 percent in less than one year (Interamerican 
Development Bank, 1989, p. 285). In addition, institutional changes are evidenced by extended controversial legislation that resulted in a new constitution -which incidentally is one of the longest of such documents in the world- that was approved in 1988 which contained numerous complex provisions that have had a tremendous impact on several social institutions in Brazil (see e.g., Pereira, 1996).

Even though Costa Rica has distinguish it self as having one of the strongest democracies in Latin America (Lehoucq, 1996), it has experienced some noticeable institutional changes in the last decade as a continued effort to counteract the negative effects left by the economic crisis of the early 1980 s including drastic inflation from $18.1 \%$ in 1980 to $90.1 \%$ in 1982 , a decline in real earnings, rising unemployment as well as underemployment (Gindling, 1993). Beginning in 1985 and aided by friendly foreign U.S. policy, Costa Rica started the privatization of state run companies, the dismantling of the state productive sector, and the creation of a private financial sector (Zarate, 1994, p. 50). More currently, the country's welfare system is being dismantled by President Jose Maria Figueres with great consequences in Costa Rican society and a profound impact in its social institutions not yet fully felt (Mesa-Lago, 1997).

By no means it is implied here that China, Sweden and the U.S. have not experienced changes as well. However, social institutional changes occurring in Sweden, USA and to a lesser extent in China have been more gradual and less disorganized than the changes taking place, for instance, in Brazil. For example, 
the Swedish general elections held in 1994 marked the return to power of the Social Democrat Party that took over the short three-year administration of a non-socialist coalition (Madeley, 1995) that experimented with a neoliberal economy. Even though the Swedish electorate has been described by Madeley (1995) as volatile rather than stable, this government transition was completely peaceful and democratically constructive in the context of a robust economy that nonetheless is being increasingly taxed by rising unemployment and financial problems for welfare policies (Svallfors, 1995). These changes in Sweden are somewhat similar to the more recent and (relatively speaking) "smooth" changes taking place in the American society today. This is in contrast to the more accelerated changes that took place in the family, gender roles, mass communication, and the economy of the U.S. beginning in the late sixties that have continued until the present time.

China's changes, on the other hand, even though have been described by some authors as "profound" (e.g., Goodman, 1994, p. ix), they have also been gradual and according to experts, positive. According to Yunling (1995), China has moved from a central planning economy to a market economy accompanied by extremely rapid economic growth. The state has shifted towards indirect management rather than direct control, and rural society is moving toward industrialization and to a "fast opening up" to the outside world. However, China still retains a Leninist political system, an institutional arrangement, a political machinery, and an economic infrastructure that is dominated by a "Communist 
party apparatus that is determined to hold on to power" (Unger \& McCormick, 1996, p. 2) that has enforced an element of stability to these changes. Indeed, when describing current changes in China, Yunling (1995) have described them as proceeding "smoothly" and far from "any drastic move toward deep-going political reform" that could cause a "chain reaction of disorder and chaos" ( $p$. 101). Therefore, the changes taking place in China could be located half way between those taking place in the Central and South American countries that were sampled in this study (i.e., Costa Rica and Brazil) and the US and Sweden.

From this "objective" overview of the institutional changes undergoing in these societies, it is thus hypothesized that participants from these five countries will differ significantly in the degree of institutional changes as well as of other Institutional characteristics perceived by the individuals within these five societies which will directly affect their youths' identity status and identity satisfaction or distress. Specifically, it is hypothesized that participants from the USA and Sweden will score higher on identity status and satisfaction than the Brazilian, Costa Rican and Chinese samples.

In this study, however, social institutions were investigated from a psychological and "subjective" perspective rather than an "objective" or sociological perspective. That is, social institutional characteristics (i.e., change, openness, accessibility, participation, support) were assessed by the evaluations individuals make of their own institutions rather than using an ecological or a structural approach commonly employed by sociologists. In this study, social 
institutions and some of the characteristics of the different social institutions were investigated by examining how individuals perceive their own social institutions. Individuals were asked to rate the extent to which certain institutions are changing and the extent to which some institutional characteristics or attributes are present in their institutions and how the different institutions and their respective characteristics impact the quality of their lives in a positive or negative way. In addition, the effects that social institutions have on identity formation were investigated by examining relations between different social institutions and some of the institutional characteristics and identity status and satisfaction.

\section{A Co-constructivist Framework for Conceptualizing and Operationalizing Identity}

\section{Formation}

The framework used for this study was derived from an ongoing program of psychosocial theory and research (Kurtines, 1984; 1987; 1996; Kurtines, Berman, Ittel, \& Williamson, 1995). This psychosocial approach is in some respects similar to other psychosocial theories proposed by theorists such as Fromm (1947), Lewin (1935), Sullivan (1953) and more recently Erikson (1964). This work, however, differs from these other psychosocial theories in that it derives from a co-constructivist perspective on human behavior and development (Kurtines, 1993). This perspective views the human species as a self-directed, goal oriented biopsychosocial organism that, like other species, is confronted with the task of successfully adapting to continuously changing conditions. Human beings, however, not only respond to changes in their 
environment, they also have the capacity to shape and influence the conditions to which they respond. The human species is a biopsychosocial organism with complex, higher order cognitive and communicative capacities that enable them to engage in decision making and problem solving activities that affect the quality of their lives. The next section will outline the view of the formation of an identity that is at the core of this co-constructivist perspective on psychosocial development.

The Formation of an Identity. The concept of identity described in this section draws on Erikson's work and the work of others (e.g., Adams, Bennion, \& Hugh, 1987; Adams \& Fitch, 1982; Grotevant \& Adams, 1984; Marcia, 1966, 1980; Marcia, Marcia, Waterman, Matteson, Archer, \& Orlofsky, 1993; Waterman, 1982, 1988, 1992; Grotevant et.al.,1982). Like Erikson, this perspective considers a sense of identity to be defined by the individual's sense of direction and purpose. Identity is the sense of who we are and what we want to do with our life. A sense of identity is the self's sense of direction and purpose. The term identity, as is frequently used in psychology, refers to a particularly mature form of self-concept, one that is characterized by a strong sense of unity, by its salience in the person's consciousness, and by its ability to anchor the person's sense of stability, individuality, and purpose (Blassi, 1993). I will describe the co-constructivist perspective on the formation of an identity in the process of outlining the three basic domains that define identity formation. 
The first domain of identity formation is personal development defined by the self's relation to its own inner reality, i.e., the self's relation to subjective reality. The domain of personal development encompasses the self's phenomenological understanding of its own subjective experiences, i.e., thoughts, moods, feelings, etc. In the process of personal development, the individual searches for, explores, and investigates available life goals and values, both actual and potential. Through this process of exploration the individual strives to identify life goals and values worthy of his/her personal commitment. A personal commitment to a core set of self chosen life goals and values serves to order, organize, and stabilize the subjective experiences of the self. A sense of identity provides an anchor point for the subjective experiences of the self through time; a sense of continuity between the self of the past and of the present; a sense that although the movement from childhood to adulthood involves developmental change, the essential self is maintained and persists.

The sense of continuity that emerges out of these encounters is defined in terms of the self's goals and values. At the early levels of development, the self's goals and values are expressed in terms of the satisfaction of immediate needs and interests and hence tend to be variable. However, with the development of a full range of competencies (including specialized competencies, abilities, capacities, resources, etc. that allow the development of modes of personal expressiveness and higher order critical decision making and problem solving skills), the individual's needs and interests become increasingly 
more defined in terms of long range goals and values, eventually including life goals and values. A life goal is the end or purpose in life that the individual aims to reach or accomplish. Values are those things that the individual considers desirable, good, right, useful, or important. With the development of a full range of cognitive and communicative competencies, the individual acquires the capacity to make decisions about the life goals and values that give direction and purpose to the individual's specialized competencies, abilities, capacities, resources, etc.

The formation of a sense of identity, I noted, involves an extended period of exploration with the goal of making a commitment to the life goals and values that give one's personal history its direction and purpose. The complex constellation of self-chosen life goals and values that the individual internalizes during the formation of an identity is the core of one's sense of identity. A commitment to the self-chosen life goals and values that are internalized during the formation of an identity facilitate the process of making decisions that shape and influence personal change. The domain of personal development is concerned with the quality of the individual's personal life. The formation of a sense of identity not only provides the self with a sense of direction and purpose, it also helps to provide the individual with a sense of continuity over time. A commitment to a core of life goals and values anchors and stabilizes the subjective experiences of the self. 
The self is also defined by its relation to the external world at a particular point in time. The self encounters two domains of external reality, intersubjective (social) reality and objective (physical/natural) reality. Both define basic identity domains. Movement through the levels of development (i.e., from childhood, preadolescence, adolescence and into adulthood) involves an expansion of the self's relation to both external domains at each developmental transition.

The second domain of identity formation is interpersonal development defined by the self's relation to others, i.e., intersubjective reality. The domain of shared mutual understanding integrates the subjective experiences of the self with others. The social reality the self encounters in the course of development includes a broader array of social relationships and new life roles as well as new life goals and values. The domain of interpersonal development is concerned with the quality of the individual's interpersonal life - the quality of our relations with others. The process of the formation of an identity is thus not a purely subjective process; it does not take place in a social vacuum. We choose the goals and values that provide the direction for our life (and we are responsible for our choices), but we do not choose them in isolation. Our decisions are made in the context of our relations with others, and our relations with others helps to define the quality of our interpersonal life. From a co-constructivist perspective, the formation of an identity involves both the subjective construction and intersubjective co-construction of life goals and values. 
The third domain of identity formation is the individual's belief about the world or world view. World view helps to define the self's relation to objective reality. The development a world view integrates the subjective experiences of the self with the external world of natural phenomena. The natural world the self encounters in the course of development includes the physical world itself and all of the phenomena to which we attribute a mind independent ontological status. Our world view includes both "factual" and "ideological" beliefs about phenomena not considered subjective or intersubjective. Our world view thus encompasses not only the factual information represented by our scientific, academic, intellectual, and technical beliefs and knowledge, but also the ideological information that is encompassed by our religious, political, moral, economic, etc. beliefs, to the extent that we consider such phenomena to be "mind independent."

From a co-constructivist perspective, the formation of a sense of identity thus involves development in three broad domains, 1) personal development, 2) interpersonal development, and 3) world view. The achievement of an identity involves exploration and commitment in each of the three domains.

Transcultural and Culture Specific Dimensions of Identity. In addition to a view of the structure of identity as comprised of three basic domains, a coconstructivist approach also hypothesizes a complex dimensionality to this structure that includes both transcultural and culture specific components. That is, a co-constructivist approach hypothesizes that some components of identity 
(e.g., the identity domains) can be characterized as transcultural, i.e., they are defined in terms of the broadest and most general cultural and historical horizons descriptive of a particular historical period. It is also hypothesized that some components of identity (e.g., content areas within the identity domains) can be characterized as culture specific, i.e., they are defined in terms of local and particular cultural and historical horizons descriptive of a particular historical period of a specific cultural tradition. A co-constructivist approach thus considers identity to have a complex and multidimensional structure defined at different levels of locality and particularity and that includes transcultural and culture specific components.

The transcultural components present human beings with challenges that transcend particular cultural traditions; the culture specific component with challenges that characterize specific cultural traditions. For instance, individuals from a culture that places high value on particular religious practices at a certain historical period will be confronted with the issue of incorporating, adapting or rejecting their religious upbringing into a coherent view of the world whereas individuals from a culture that does not emphasize religion as part of their daily life may or may not have to deal with religious issues at all during the consolidation of their identity. A co-constructivist approach thus views the challenge that the formation of an identity presents as comprised of two major tasks. The first is that of adapting to the broad and general type of challenges 
that are present in all cultures (e.g., those related to personal development, interpersonal development, and beliefs about the world), the types of broad challenges that define the three identity domains. The second task is that of resolving the challenges that arise out of the specific cultural/historical matrices that define particular cultural traditions, the types of challenges that define the specific content areas within the identity domains.

The research to be conducted as part of this dissertation sought to extend the literature on identity not only by investigating how culture influences the process of identity formation with cross-national/cultural samples but also by investigating cross-culturally the role that social processes play in identity formation. Social processes that influence identity formation were investigated at two distinct levels. At one level, the study examined the effects of ethnic/cultural variation on the process of identity formation between ethnic/cultural groups. At a second level the study investigated the effects of social institutions on identity formation within ethnic/cultural groups.

Goals of the Proposed Study

The proposed study had three main goals. The first goal of this study was methodological, and sought to make a contribution by refining a measure of social institutional change. More specifically, this study involved the first large scale use of the SIR (Social Institutions Rating) in a cross-national/cultural study 
that examined how this measure performs with participants from different ethnic/cultural groups.

The second goal draws on the small but growing psychological literature on the impact of culture on identity. To begin to explore the impact of culture this study examined the effects of ethnic/cultural variation on identity formation between groups of late adolescents from six sites from five countries (USA, Sweden, China, Costa Rica and Brazil) representing different linguistic and ethnic/cultural traditions.

The third goal of this study draws on the sociological literature on social institutions to begin to explore the empirical relationship between social institutions and identity formation. Specifically, this study examined the relationship of institutional attributes (including institutional change, openness, participation, access and support) and identity formation within these six ethnic/cultural traditions experiencing different degrees of institutional change in the context of varied sociocultural conditions.

Method

\section{Participants}

The participants were 1010 college students from six sites representing five countries: Brazil, China, Costa Rica, Sweden, and the USA. Participants from Brazil $(n=165)$ were attending the Federal University of Rio Grande at Porto Alegre (Rio Grande is the southernmost state of Brazil). Participants from China 
were recruited at the Nanjin Normal University in the province of Jiangsu and at the Jiangxi Normal University in Nanchang, Jiangxi $(n=121)$. Students from Costa Rica $(n=130)$ were attending the University of San José, a small private university in the capital of this Central American country. Students from Sweden ( $n=91$ ) were attending Uppsala University, a mid-sized public university located southeastern Sweden. Lastly, two sites were sampled in the US; one at the University of Nebraska at Lincoln $(n=229)$ and the other one at Florida International University in Miami $(n=274)$. Even though both samples from the US are from public and large sized universities they are located in different geographic regions in the US and have different ethnic compositions. The sample from FIU consisted mainly of students of Hispanic background (70\%) while the sample from Nebraska was comprised of mainly Anglo students (84\%). Consequently, the sample from the US was reduced in size because only Hispanics from Miami $(n=192)$ and Anglos from Nebraska $(n=193)$ were used in subsequent analyses in order to do cross-cultural comparisons among sites. The final sample, thus, was reduced $11.68 \%$ of its original size for a total of 892 participants. All participants were offered extra class credit for their participation.

The samples differed on several demographic variables (age, gender, grade, socioeconomic and marital status). Specifically, there were differences among sites in the distribution of gender, $X^{2}(5, N=875)=67.19, \underline{p}<.001$, years of schooling (first two years versus remaining years of school), $X^{2}(5, N=864)=$ 
$118.05, \underline{p}<.001$, and marital status (single versus other than single), $x^{2}(5,863)=$ $27.67, \underline{p}<.001$. To determine which groups were accounting for the significance in the Chi-square tests, adjusted standardized residuals were calculated for each cell. Adjusted standardized residuals are observed minus expected values divided by an estimate of their standard error (the square root of the expected value for each cell), normalized into $z$ scores; thus a value of \pm 1.65 signifies 1.65 Standard Deviation (SD) above or below the expected value for that cell based on the distributions among the entire sample (see Haberman, 1978). Any adjusted standardized residual greater than 1.7 was considered significantly stable to be reported in subsequent results when Chi-Square tests are performed.

The distribution of gender, grade, and marital status with their respective adjusted standardized residuals are listed in Table 1,2, and 3, respectively. Adjusted standardized residuals indicated that there were more males than expected in Nebraska, Brazil, and Costa Rica (Table 1). Conversely, there were more females than expected in Miami and Sweden. China had similar numbers of males and females compared to the rest of the sites. There were more students in their last years of school than expected in Costa Rica, Sweden and Miami (Table 2). Conversely, there were more students in their first two years of school than expected in Nebraska, Brazil and China. More singles than expected were found in Miami, Nebraska, Brazil, and China (Table 3). In contrast, the sample from Costa Rica included a disproportionate number of 
married students. Out of the six sites, $61 \%$ of the students who reported a marital status other than single were found in the Costa Rican sample alone. Sweden had similar number of singles and non-singles compared to the rest of the sites (see Table 3).

The sites also differed on reported age, $F(5,853)=101.85, \underline{p}<.001$, and SES, $F(5,863)=27.67, p<.001$. Scheffe post-hoc analysis of mean differences revealed that students from Costa Rica were older $(\bar{x}=29.10, S D=8.07)$ than the rest of the sites which may explain why they were also more likely to report a marital status other than single. Students from Sweden were older $(\bar{x}=22.57$, $\mathrm{SD}=4.35)$ than students from China $(\bar{x}=19.54, \mathrm{SD}=1.29)$ and Miami $(\bar{x}=19.46$, $S D=3.49)$. Students from Brazil $(\bar{x}=21.00, S D=4.56)$ were older than students from Nebraska $(\bar{x}=19.29, S D=1.47)$. Students from Miami, Nebraska, and China, however, did not differ from one another in age. Nevertheless, they differed in reported SES as indexed by a self-report scale with values ranging from 1 (lowest) to 7 (highest), with students from Nebraska and Miami reporting higher SES than the rest of the sites $(\bar{x}=4.53, S D=1.12$ and $\bar{x}=4.48, S D=1.18$, respectively). Conversely, Chinese students reported the lowest SES ( $\bar{x}=3.16$, $S D=1.34)$. Students from Costa Rica, Sweden, and Brazil, however, did not differ on SES from one another $(\bar{x}=3.71, S D=1.21 ; \bar{x}=3.95, S D=1.23$ and $\bar{x}=4.02$, $\mathrm{SD}=.93$, respectively). Anglos and Hispanics from the US were not significantly different from each other with regards to SES.

Measures 
Two measures were utilized in the study: the Identity Domain Survey (IDS) and the Social Institutions Rating (SIR).

The Identity Domain Scale (IDS). The Identity Domain Scale (Schmaltz, Kurtines, Berman, \& Briones, 1994) was used to assess identity status and identity satisfaction and distress which generate the primary dependent variables for the proposed study. The IDS is a group administered, self-report measure, that uses self-chosen codes as well as open-ended written responses (Appendix A). It contains 54 items that tap three main domains of identity: personal development, interpersonal development, and world view. Each of these three domains is comprised of three content areas for a total of 9 content areas including career goals, sense of self, and long-term life goals as part of the personal development domain; friendships, belonging to groups, gender and sexuality issues as part of the interpersonal development domain; and religious, moral, and political issues as part of the world view domain. Within each of the nine content areas, the participant is asked to identify the most good or positive and distressful experiences from a list. For each of the positive and troublesome experiences, the subject uses a five-point Likert-type scale to rate the degree to which the experience was positive (1=Slightly Positive, 2=A Little Positive, 3=Positive, 4=Very Positive, 5=Extremely Positive) and the degree to which the experience was distressful (1=Slightly Distressful, 2=A Little Distressful, 3=Distressful, 4=Very Distressful, 5=Extremely Distressful). Subsequently, if the 
subject responds "extremely distressful", s/he is asked if the distress has lasted longer than three months.

In addition, to provide an assessment of identity status within each content area, the IDS includes 4 items in the form of statements from which the subject has to choose only one of them. These 4 items contains four prototypical "status" statements that characterizes each status (Appendix A). The participants are asked to select the statement that best describes themselves.

Scoring the IDS. The IDS yields two sets of quantitative scores: satisfaction and distress scores as well as status scores for each of the nine content areas and for the three domains. The total Identity Satisfaction Score consists of the average of the satisfaction and distress ratings for each of the nine content areas (Career Goals, Sense of Self, Long Term Life Goals, Friendships, Belonging to Groups, Gender and Sexuality Issues, Religion, Morality, and Political Issues) using a reversed scoring procedure. Thus, the Distress items are assigned a reversed value (i.e., 5 becomes 1, 4 becomes 2, 2 becomes 4 , and 1 becomes 5 ) to be added to the Positive items so a continuos bipolar score can be generated. For instance, if a subject chooses a 4 on a positive rating item (4=Very Positive) and a 1 for the same content area on the distressed rating ( $1=$ Not Distressed), 1 on the distressed rating is reversed to 5 in order to generate an added satisfaction score of 9 (mostly satisfied). Conversely, if a participant chooses a 1 on the positive rating ( $1=$ Not Positive) 
but a 4 on the distress rating ( $4=$ Very Distressful), 4 is reversed to 2 in order to generate an added satisfaction score of 3 (3=Mostly Distressed). Using a reverse scoring procedure with the IDS creates a bipolar satisfaction score ranging from 2 to 10 (2=Extremely distressed, 3=Very distressed, 4=Moderately distressed, 5=Slightly distressed, $6=$ Neither distressed nor satisfied, $7=$ Slightly satisfied, $8=$ Moderately satisfied, $9=$ Very satisfied, 10=Extremely satisfied). The three Domain Satisfaction Scores (Personal, Interpersonal, and World View) consists of the average of the added satisfaction and distress ratings for each of the 3 content areas within each domain using also the same reversed scoring procedure described above. The total satisfaction score can also be seen as the average of the added satisfaction and distress ratings for all of the three identity domains.

Internal consistency coefficients (alpha) were computed for the Total Identity Satisfaction Score as well as for the Personal, Interpersonal, and World View Satisfaction score for each site (Table 4). The Total Identity Satisfaction score showed good internal consistency within the samples ranging from $\propto=.55$ (in Sweden) to $\propto=.76$ (in Miami). The Personal and Interpersonal Identity Satisfaction scales were also moderately internally consistent with the exception of the sample from Sweden in the Personal domain $(\alpha=.42)$ and the sample from China and Miami in the Interpersonal domain $(\alpha=.38$ and $\propto=.47$, respectively). The rest of the alphas were acceptable $(\propto=.52$ to $\propto=.72)$. It has to be pointed out that these alphas are adequate for research purposes 
considering that the scales by domain consisted of only 3 items each. As it can be noted (Table 4), the World View Satisfaction scale showed the lowest internal consistency of the scales from the IDS ranging from $\propto=.38$ (in China) to $\propto=.50$ (in Miami), with the exception of the Swedish sample that exhibited not cohesiveness at all in responses $(\alpha=.03)$.

Exploratory factor analyses with varimax rotation were performed for the IDS within each site (see Appendix C). A three factor solution showed that the items in the personal domain tended to load together across sites while the other two theoretical scales showed more variability. The theoretical scoring was retained, however, because the theoretical scales yielded adequate alphas in most sites. These results indicate that although the IDS has adequate psychometric properties, additional instrument development is recommended to improve the factor structure of the measure and the reliability of the scales.

The IDS also yields an Identity Domain Status Score (IDSS) for each of the identity domains (Personal, Interpersonal, and World View). The Content Area Status Scores are derived from the value assigned to the statement the individual selects from the four statements provided for that content area. The values attributed to the status statements are as follows: 1=Diffused, 2=Foreclosure, 3=Moratorium, 4=Achievement. The four statements provided for each content area characterize the four identity statuses (achieved, moratorium, foreclosed, and diffused). The Identity Domain Status Scores are based on the subject's modal status score for the three content areas within 
each domain. In other words, if a subject has the same status value for all three content areas (e.g., 4, 4, 4, or 3, 3, 3, etc.), then that value is the Identity Domain Status Score. If a subject has two of the same values for the three content areas (e.g., 4, 4, 2, or 2, 2, 3, etc.), then that modal value is the Identity Domain Status Score. Finally, if the subject has three different status values (e.g., 4, 1, 3, or 2, 4,1 , etc.) then the Identity Domain Status Score is coded as Undifferentiated. The Social Institutions Rating (SIR).

The Social Institutions Rating (SIR; Briones \& Kurtines, 1994) was used to generate one of the independent variables for the study. The SIR is a group administered, self-report measure that uses a 5 point Likert scale format as well as open-ended responses (see Appendix B). The SIR is a factorially derived, two dimensional measure that participants use to provide a report on the presence or absence of five institutional attributes (change, openness, access, support, participation) and an evaluation (negative versus positive effect of these attributes on the quality of their lives) regarding their society's primary social institutions. The original nine social institutions evaluated in the SIR are: 1) The Family, 2) The Government, 3) Mass Communication, 4) Gender Roles, 5) Religion, 6) Educational Institutions, 7) Economic Institutions, 8) Political Institutions, and 9) Judicial/Legal Institutions). The five institutional attributes assessed are the degree of: 1) change, 2) openness, 3) supportiveness, 4) accessibility, and 5) participation in the changes. In this study, however, mass communication, educational and economical institutions were not included for 
purpose of scoring and further analyses given that these institutions showed a minimal or sometimes redundant contribution to the explained variance in participants' response compared to the other institutions as revealed by confirmatory factor analyses within each site. Consequently, the social institutions that were used in the rest of the analyses were 1) The Family, 2) The Government, 3) Gender Roles, 4) Religion, 5) Political and 6) Legal/Judicial Institutions. A noteworthy exception in the scoring procedure was made with the Chinese sample. Participants in China were not allowed to rate their Government nor their Political institutions and thus these two institutions were not included in the Chinese version of the SIR. In order to create compatible scores with the rest of the sites, therefore, the scoring of the SIR for the Chinese sample only included 4 social institutions: 1) The Family, 2) Gender Roles, 3) Religion, and 4) Legal Institutions.

Scoring the SIR. The SIR yields two types of scores: an attribution score and an evaluation score. The Social Institutions Attribute Score consists of the averaged sum of the subject's attribution (degree of presence or absence) of each of five institutional attributes (change, openness, accessibility, support, and participation) for each social institution. The social institutions attributes scale provides a score ranging from one to five ( $1=$ Not present, $5=$ Highly Present) for each of the six institutions (except in China where participants evaluated 4 instead of 6 institutions). The social institutions attribute score can also be added and averaged across the six institutions (four in China) to create an 
Overall Institutional Attributes scale with scores ranging from 1 to 5 (1=Not present, $5=$ Highly present).

The Evaluation Score consists of the average of the participants' evaluation (positive or negative impact) that the four institutional attributes have on the quality of their lives and a satisfaction score for each institution. The four institutional attributes are institutional change, openness, accessibility, and support. The institutional evaluation score can also be added and averaged across the six institutions (four in China) to create an Overall Institutional Evaluation scale using a reversed scoring procedure similar to the one previously described for the IDS, with scores ranging from 2 to 10 (2=Extremely negative impact, $3=$ Very negative impact, $4=$ Moderately negative impact, $5=$ Slightly negative impact, $6=$ Neither negative nor positive impact, $7=$ Slightly positive impact, $8=$ Moderately positive impact, $9=$ Very positive impact, $10=$ Extremely positive impact).

\section{Procedure}

The IDS and the SIR were translated by native speakers of 4 languages from the original English version. The four languages are Chinese, Spanish, Swiss and Portuguese. Furthermore, both measures translated into these four languages were translated back into English again by independent translators who were never exposed to the original English versions of both measures in order to ensure a correct and valid translation. This procedure is the standard practice used for translation and validational purposes. 
The IDS and the SIR were administered in group classroom settings. Instructions were given verbally as to the format of the measures. The instructions given were also written on the measures. The instructions for the IDS are as follows:

"You have been given, the Identity Domain Scale. We are interested in your thoughts and feelings about a variety of issues relating to your identity. Identity has to do with your sense of self. More specifically, your satisfaction or dissatisfaction with the direction of your life. This survey has nine parts. Each part asks about a different area of identity. You will be asked to describe what is good or positive and what is distressful or troublesome in each area. You may write directly on the extra sheets provided but not on the survey. There are no right or wrong answers. Thus, please be as truthful as possible. You may write in either pen or pencil. Please do not skip any question. If the question does not apply to you please indicate this in the space provided."

The instructions for the SIR are as follows:

"You have been given the Social Institutions Rating. This survey asks questions about social institutions in your society. Some of the social institutions are the family, the government, gender roles, religion, mass communication, educational, legal, economic, and political institutions among others. You will also be provided with specific examples for each of these institutions. This survey asks about your satisfaction with these 
institutions, how much change they are undergoing, how satisfied you are with the change, how much the change affects your life, and how much you participate in the changes that are taking place. There are no right or wrong answers. Thus, please be as frank as possible. Please write your answers on the bubble sheets provided. You may write in either pen or pencil. Please do not skip any question. If the question does not apply to you please indicate this in the space provided on the extra sheets."

\section{Results}

\section{Psychometric Analyses}

The first goal of this study was to examine the psychometric properties of the SIR.

Factor Analysis. This section describes an exploratory factor study of the SIR in each of the six sites. The focus of this study was on the five evaluation ratings (change, openness, accessibility, support, and satisfaction) and the five attribution ratings (degree of change, openness, accessibility, support, and participation) across all nine institutions for a total of ten ratings. For the first analyses, all 10 ratings were subjected to a principal components analysis with a varimax rotation (see Tables 5 through 10). The principal components analysis of 10 ratings yielded two factors with an eigenvalue greater than 1.0 in all sites but in Miami that yielded a three factor solution. In the sample from Miami, however, the third factor accounted for a small portion of the total variance $(11.0 \%)$ and thus was collapsed in two factors instead. 
The total percent of the variance accounting for a two factor solution ranged from $54.5 \%$ in China to $68.2 \%$ in Costa Rica. The first factor of the SIR accounted for the largest proportion of variance, ranging from $29.5 \%$ of the total variance $\left(e^{1}=2.95\right)$ in China to $47.4 \%$ in Sweden $(e=4.74)$. Factor 2 accounted for a smaller proportion of variance, ranging from $16.8 \%$ in Sweden $(e=1.68)$ to $25.8 \%$ in Brazil $(e=2.58)$. These results thus show a two factors solution, one factor with all of the evaluation ratings with high loadings on this factor even though two of the attribution ratings (Institutional Access and Support) also loaded on this factor in most sites, except in China where the two factors solution more clearly differentiates between an evaluation and an attribution factor (see Table 9). The second factor was an attribution factor, with at least three of the attribution ratings (Institutional Change, Openness and Participation) with high loadings on this factor, and none of the evaluation ratings loading on this factor. The only exception to this was in Sweden where only two of the attribution ratings (change and participation) loaded in the second factor (Table 10). As Table 5 through 10 indicate, the overall results of the factor analyses in each site provide evidence that the empirical structure of the evaluation and attribution ratings clearly represent a two dimensional structure of the SIR: one that describes the characteristics or qualities (attributes) present in a particular social institution and the other, one that evaluates the extent to which these

\footnotetext{
${ }^{1}$ e=eigenvalue
} 
institutional attributes have a positive or negative impact in the quality of the individual's life.

Item Analysis. An item analysis was conducted on the 10 scores for the SIR that included item total correlations, squared multiple correlations, scale Alphas with the items deleted, scale means with the item deleted, and scale variance with the item deleted (cf. Noruis/SPSS, 1988). The results indicated that none of the Alpha coefficients for any of the scales would be significantly increased by removing any of the items.

Internal Consistency Reliability. Internal consistency reliability was estimated for the Evaluation and Attribution scores across the institutions as well as for the Attribution score for each social institution within each site using the Alpha coefficient. Within each site, the Overall Institutional Attribute scale (across institutions) showed good internal consistency (alpha) ranging from alpha $=.63$ (in Sweden) to alpha=.80 (in Nebraska). Table 11 includes the internal consistency of the Overall Institutional Attributes scale for each site. The overall Institutional Evaluation scale (across institutions) also showed good internal consistency within each site (Table 11) ranging from alpha=.62 (in Sweden) to alpha=.87 (in Costa Rica). The institutional attribute scale within each social institution showed an internal consistency that ranged from alpha= .47 for Gender Roles in China to alpha $=.85$ for Religious institutions in Nebraska (Table 12). Finally, the institutional evaluation scale within each social institution 
showed an internal consistency that ranged from alpha $=.47$ for Gender Roles in

China to alpha $=.85$ for Religious institutions in Nebraska (Table 13).

Hypotheses Testing

The second goal of this study was to examine the effects of ethnic/cultural variation on identity formation between groups of late adolescents from five countries (USA, Brazil, Costa Rica, China, and Sweden) representing six different linguistic and ethnic/cultural traditions (including Hispanics and Anglos from the US). This section describes the analyses for testing the hypotheses with respect to the effects of ethnic/cultural variation on identity formation. Hypothesis 1 states that participants in the six ethnic/cultural groups will differ significantly on the three main domains of identity status (personal development, interpersonal development, and world view) assessed by the IDS, with participants from the U.S. and Sweden showing more frequently an achieved and moratorium status than participants from the remaining sites (Brazil, China, and Costa Rica).

Because of the categorical nature of the identity status scores, the statistical analyses used to test Hypothesis 1 consisted of several Chi-Square tests comparing the frequency with which the identity statuses are represented among the six cultural groups with a significance level set at .05 . Before conducting Chi-Squares, analyses across sites were performed to establish whether a relationship existed between demographic variables and identity statuses within each domain given that the samples differed in gender, grade, 
and marital status composition, as well as by age and SES. These analyses were conducted in an effort to further statistically control for any influence the demographic variables may have on identity status.

Participants' age, marital status, and SES were not related to any of the identity status by domain (personal, interpersonal, and world view) across sites. In addition, Personal, Interpersonal, and World View Status were independent of gender and grade with one exception. Personal identity status was not independent of grade. There were more Achieved participants in the personal domain in their last 2 years of school than expected, $X^{2}(4, N=830)=10.08, \underline{p}$ $<.05$. Therefore, Chi-Square analyses were performed using grade (first versus last 2 years of school) as an additional layer of control variable in the personal identity domain. Doing this type of cross tabulation (site $\mathrm{x}$ identity status $\mathrm{x}$ grade), however, created more than $20 \%$ of the cells with an expected value less than 5 , not only because there were not enough Diffused participants in the last years of school within each site but also because there were not enough participants in their last two years of school in China and in Nebraska.

In an effort to continue controlling for grade and reduce the number of empty cells, grade categories were collapsed into first versus other than first year of school. In addition, the four identity statuses in the personal identity domain were collapsed into Explored (moratorium and achieved) versus Non-Explored statuses (diffused and foreclosed). This type of cross-tabulation (identity status $x$ site) with one layer of control variable (grade) was performed for the personal 
identity domain only because the interpersonal and world view status were not related to grade nor to any of the other variables. Significant differences among sites continued to emerge even after controlling for grade. For purpose of clarity, thus, Chi-Square tests were performed with the four identity statuses without the additional layer of grade.

Personal Development Status. There were more participants classified in the Explored status (moratorium and achieved) in Nebraska and Costa Rica compared to the rest of sites, $X^{2}(5, N=386)=39.11, \underline{p}<.001$, within their first year of school. Nebraska and Costa Rica, however, did not differ from each other, $X^{2}(1, N=131)=2.54, p=.11$. Conversely, there were more Non-Explored participants (diffused and foreclosed) in China and Sweden (Table 14). (Note: Participants who were not classified in any of the four theoretically derived statuses were excluded from further analyses). Further Chi-square tests revealed that more participants were classified in the Non-Explored status (diffused and foreclosed) in Sweden than in China, $X^{2}(1, N=78)=8.50, \underline{p}<.01$. As hypothesized, more Explored statuses were found in Miami and Nebraska, and more Non-Explored statuses in China and Brazil. The distribution of statuses for the Costa Rican and Swedish sample, however, was contrary to the hypothesized direction of the differences. Participants from Costa Rica were expected to be classified more often in the Non-Explored statuses and participants from Sweden in the Explored statuses. Brazil and Miami had similar number of explored versus non explored participants compared to the rest of the 
sites. Hypothesis 1, thus, was partially confirmed among participants in their first year of school.

In other than their first year of school, there were more Explored participants than expected in Miami, Nebraska, and Costa Rica, $X^{2}(5, N=305)=$ 45.68, $\mathrm{p}<.001$. Miami, Nebraska and Costa Rica, however, did not differ from one another, $x^{2}(1, N=214)=5.28, p=.07$. Although it was hypothesized that participants from Miami and Nebraska would be classified more often in the Explored statuses, the higher frequency of Explored statuses in the Costa Rican sample was not anticipated. Conversely, there were more Non-Explored participants in Brazil, China, and Sweden. Further Chi-square tests revealed more Non-Explored participants in Sweden than in Brazil or China. The NonExplored frequencies found in China and Brazil confirmed hypothesis 1 with regard to the expected direction of the differences in identity statuses. However, the higher frequency of Non-Explored participants in Sweden was not anticipated. Table 14 includes the distribution of Explored versus Non-Explored status in the Personal domain by site and with adjusted standardized residuals for each cell.

Interpersonal Development Status. Participants from Costa Rica, China, and Sweden were classified in the Diffused status more often than expected, $X^{2}(15, N=589)=123.40, p<.001$, compared to the rest of the sites (Table 15). No differences were found, however, when Costa Rica, China and Sweden were compared to one another, $X^{2}(2, N=318)=2.78, p=.25$. More Foreclosed 
participants were found in Nebraska and Brazil, $X^{2}(15, N=589)=123.40, p<.001$. Nebraska and Brazil, however, did not differ from each other, $X^{2}(1, N=358)=.62$, $\mathrm{p}=.43$. In addition, more participants were classified as Moratorium in China and more as Achieved in Miami and Costa Rica, $X^{2}(15, N=589)=123.40, p<.001$. Miami and Costa Rica, though, did not differ from each other, $X^{2}(1, N=306)=.45$, $\underline{p}=.50$. Again, the direction of hypothesis 1 was partially confirmed in the interpersonal domain. As hypothesized, more Diffused status were found in Costa Rica and China, and more Foreclosed in Brazil. A striking finding was the higher frequency of a Non-Explored status (i.e., Diffused) found in the Swedish sample and the proportion of individuals classified in the Moratorium status in China and Achieved in Costa Rica. Table 15 includes the distribution of the identity statuses in the Intersonal domain by site and with adjusted standardized residuals for each cell.

World View Status. As hypothesized, participants from Costa Rica and China were classified in the Diffused status more often than expected compared to the rest of the sites, $X^{2}(15, N=556)=104.31, \underline{p}<.001$ (Table 16). In addition, more Achieved participants were found in Miami. Further Chi-Square analysis between Costa Rica and China showed more Diffused participants in China than in Costa Rica, $X^{2}(1, N=229)=6.12, p<.01$. Contrary to the direction of the hypothesized differences in identity status, more Foreclosed were found in Sweden and more Moratorium in China, $X^{2}(15, N=556)=104.31, \underline{p}<.001$. Table 
16 includes the distribution of the identity statuses in the World View domain with adjusted standardized residuals for each cell and by site.

In sum, hypothesis 1 which predicted differences among sites in the Personal, Interpersonal, and World View Status was confirmed. The distribution of the status among sites, however, was more complex than hypothesized. In the Personal Development domain, more Explored status (Moratorium and Achieved) than expected were found in Nebraska and Costa Rica and more Non-Explored status (Diffused and Foreclosed) in China and Sweden, with Sweden having more Non-Explored participants than China. There were more Diffused participants than expected in Costa Rica and China in both the Interpersonal and World View domain. In addition, more Diffused than expected were also found in Sweden in the Interpersonal domain. More Foreclosed were found in Nebraska and Brazil in the Interpersonal domain and more Foreclosed in Sweden in the World View domain. There were more Moratorium than expected in China in both the Interpersonal and World View Domain. Lastly, more Achieved participants were found in Costa Rica and China in the Interpersonal Development domain and more Achieved in Miami in the World View domain. The only noticeable consistency found in identity status across the three domains was observed for the Chinese sample. Chinese participants were consistently classified in the Diffused status in the personal, interpersonal and world view more often than expected. 
Hypothesis 2: Participants in the four ethnic/cultural groups will differ significantly on the three main domains of identity satisfaction/distress (personal development, interpersonal development, and world view) assessed by the IDS, with participants from the U.S. and Sweden reporting higher identity satisfaction than participants from the remaining sites (Brazil, China, and Costa Rica).

Before testing this hypothesis, however, separate bivariate correlations and Analysis of Variance (ANOVAs) were performed to establish whether a relationship existed between any of the demographic variables and identity satisfaction/distress in the three identity domains. These analyses were conducted in an effort to rule out any influence the demographic variables may have on identity satisfaction or else to statistically control for them. Grade was the only demographic variable not related to identity satisfaction in any of the three domains. The rest of the demographic variables were either covaried or entered as additional factors in the analyses. Because of the multiple dependent variables, the statistical analyses used to test Hypothesis 2 consisted of Multivariate Analysis of Co-Variance (MANCOVA). The dependent variables were the 3 Identity Satisfaction scales from the IDS [1) Personal Development, 2) Interpersonal Development, and 3) World View)]. The independent variables were site with six levels (Miami, Nebraska, Brazil, Costa Rica, China, and Sweden) in addition to gender with two levels (males and females). Gender was used as an additional factor in the MANCOVA in order to examine any possible 
interaction between gender and site. Marital status was dummy coded $(0=$ single, $1=0$ ther than single) to be covaried in the analysis together with age and SES. Marital status was not used as an additional factor in the MANCOVA because there was at least one empty category for the Chinese sample, namely, other than single. (Note: College students in China are not allowed to get married and study at the same time. That is why there are only singles in the Chinese sample).

Multivariate effects were significant for site, $F(15,2205)=12.09, \underline{p}<.001$. (Note: Pillai's trace criterion F approximation is reported for these and subsequent MANOVA or MANCOVA findings). Even though a significant interaction was found between site and gender at the multivariate level, $F(15$, $2205)=1.70, \underline{p}<.05$, the interaction of the tests at the univariate level did not reach significance at the .05 level in any of the three identity domains. Further examination of the univariate F-tests for site, however, revealed that there were significant differences in the three identity domains over and above the combined effect of the demographic variables (gender, marital status, age and SES). Specifically, differences were found in the Personal Development Satisfaction rating (See Table 17), $F(14,749)=7.51, \underline{p}<.001$, with students from Costa Rica reporting the highest satisfaction $(\bar{x}=7.57, S D=1.12)$ followed by students from Brazil $(\bar{x}=7.32, S D=1.17)$, Miami $(\bar{x}=7.17, S D=1.37)$ and Nebraska $(\bar{x}=6.96, S D=1.29)$. The lowest satisfaction was reported by students from China $(\bar{x}=6.50, S D=.92)$ and Sweden $(\bar{x}=6.63, S D=.92)$. 
Scheffe's method of post-hoc multiple mean comparisons revealed that the mean satisfaction reported by students from China and Sweden differed significantly from those reported by students from Miami, Brazil, and Costa Rica but not from each other. In addition, the satisfaction of students from China also differed from those of Nebraska's which in turn differed from those of Costa Rica's. (Note: Scheffe's method is reported for this and subsequent post-hoc multiple mean comparisons. This method was chosen because it is the most conservative test that performs simultaneous joint pairwise comparisons for all possible pairwise combination of mean using the $\mathrm{F}$ sample distribution and adjusting the level of significance according to the number of groups being compared). As hypothesized, there were differences in identity satisfaction/distress among the six sites in the personal domain. The highest satisfaction in the personal domain reported by participants from Costa Rica and Brazil, however, was not expected. Similarly, the lower satisfaction of participants from Sweden was not anticipated either. The lowest satisfaction of Chinese students, however, partially confirmed hypothesis 2 with regard to the direction of the differences in identity satisfaction.

Satisfaction/Distress in the Interpersonal Development domain was also significantly different among sites, $F(14,749)=10.26, \underline{p}<.001$, with students from Brazil reporting the highest satisfaction $(\bar{x}=8.11, S D=1.12)$ followed by Nebraska $(\bar{x}=7.60, S D=1.09)$, Miami $(\bar{x}=7.59, S D=1.16)$ and Costa Rica $(\bar{x}=$ $7.42, S D=.93)$. The direction of the hypothesized differences was confirmed by 
the lowest satisfaction reported by students from China $(\bar{x}=6.55, S D=.84)$.

Unexpectedly, however, Sweden followed China in terms of the lowest satisfaction among sites $(\bar{x}=7.17, S D=.92)$ and Brazil reported the highest satisfaction of all. Multiple mean comparisons revealed that the satisfaction of Brazilian and Chinese students differed significantly from rest of the sites and from each other. Satisfaction of students from Nebraska, Miami, Costa Rica, and Sweden, however, did not differ from one another which again partially confirmed hypothesis 2 with regard to the direction of the differences. As hypothesized, Chinese students reported the lowest satisfaction.

Similarly, differences in satisfaction were found in the World View domain, $F(14,749)=8.93, \underline{p}<.001$, with students from Brazil reporting the highest satisfaction $(\bar{x}=7.34, S D=.88)$ followed by Miami $(\bar{x}=7.30, S D=1.03)$, Nebraska $(\bar{x}=7.21, S D=.99)$, and Costa Rica $(\bar{x}=6.94, S D=1.01)$. As shown in the personal and the interpersonal domain, students from China reported the lowest satisfaction in the world view domain $(\bar{x}=6.35, S D=.80)$, partially confirming the direction of the hypothesized differences. Consistent with the findings in the personal and the interpersonal domain but contrary to the direction of the hypothesized differences, students from Sweden reported lower satisfaction than the rest of the sites $(\bar{x}=6.47, S D=.58)$. Multiple mean comparisons revealed that the satisfaction of students from China and Sweden differed significantly from the rest of the sites but not from each other. Furthermore, World View satisfaction of students from Costa Rica differed from the satisfaction of students 
from Brazil. Participants from Miami and Nebraska, however, did not differ from each other or from participants in Brazil and Costa Rica which again partially confirmed hypothesis 2 in the world view domain. Table 17 lists the mean, standard deviation, and sample size for the Personal, Interpersonal, and World View Satisfaction/Distress ratings.

In sum, hypothesis 2 which stated that Identity Satisfaction/Distress would differ among the different sampled sites was confirmed. The direction of these differences, however, was more complex than expected. Consistently across the three identity domains, participants from China and Sweden reported the lowest Identity Satisfaction. Satisfaction in the Personal development domain was highest in Costa Rica and Brazil followed by the two sites in the U.S. (Miami and Nebraska). Satisfaction in both the Interpersonal and World View was highest in Brazil and the U.S. followed by Costa Rica.

The third goal of this study was to examine the empirical relationship of social institutions and identity formation within the six ethnic/cultural traditions. This section describes the results from testing the hypotheses with respect to the relationship of social institutions and identity status and satisfaction within the different ethnic/cultural groups.

Hypothesis 3: Social Institutional change, openness, accessibility, participation, and support as indexed by the Overall Attribution Scale of the SIR are predictive of Identity status on the three main Identity Domains (Personal, Interpersonal, and World View) as assessed by the IDS. 
Because of the continuous nature of the SIR scores on social institutional change, openness, accessibility, and support and the categorical nature of the Identity Status Scale of the IDS, separate discriminant analyses for each domain were used to investigate whether any of the institutional attributes predicted identity status within each site. Before running discriminant analyses, however, separate correlation matrices were performed to establish whether a relationship existed between the demographic variables and identity status for the three identity domains in each site. If any of the demographic variables were related to identity status, they would be entered as predictors in the discriminant analyses (in addition to the institutional attributes) in order to examine the possible individual or combined effect these variables may have on identity status for each identity domain.

Age, gender, SES, marital status, and grade were not related to identity status in the personal, interpersonal or world view domain in Costa Rica nor in Sweden. In the remaining sites, however, some significant relations emerged. When discriminant analyses were performed within each site, therefore, the demographic variables that were significantly related to identity status in any of the three domains were entered in the discriminant functions in order to evaluate their contribution in predicting identity status in addition to the institutional attributes. When applicable, gender was dummy coded (1=male, $2=$ female $)$ in order to be covaried with the rest of the continuous variables (age, grade, SES, and the institutional attributes) in the subsequent analyses. 
Stepwise discriminant analyses were performed within each site. This is the method of choice when it is not known how well the proposed variables discriminate between the groups (Klecka, 1980). The stepwise method enters the variables into the predictive equation, one at a time, with the strongest discriminator going in first. Hypothesis 3 was not confirmed in the personal nor the interpersonal domain. No variables were qualified for the analyses (having a minimum partial $F$ of 3.84 ) within any of the sites. That is, none of the institutional attributes (or any applicable demographic variable) significantly discriminated among the identity statuses in any site. Nevertheless, some institutional attributes and one demographic variable showed significant discriminant ability among the statuses of the World View domain in Nebraska, Brazil, and China. Tables 9,10, and 11 present the results for Nebraska, Brazil and China respectively.

Nebraska. The multivariate aspects of the model can be examined by using the canonical discriminant functions (Hair, Anderson, \& Tatham, 1987). Only one out of six independent variables (SES, institutional change, openness, participation, accessibility, and support) was sufficient to account for the variance in the discriminat function, namely, Institutional Support, $X^{2}(3, N=133)=12.53$, p $<.01$; the rest of the variables washed out in the analysis. The canonical correlation for the discriminant function in the sample from Nebraska was .304 , and by squaring the correlation it can be seen that $9 \%$ of the variance in the person's identity status can be explained by the model that contained 
Institutional Support. (Note: Institutional Support is the average of perceived support across six social institutions: the family, government, gender roles, political, legal, and religious institutions).

Group centroids can be used to interpret the discriminant function from an overall perspective (Hair et al., 1987). A group centroid is reported (see Table 18) because it is "the imaginary point which has coordinates that are the group's mean on each of the variables" (Klecka, 1980, p. 16). They represent the mean of the individual Z-scores for each group. As can be seen in Table 11, the groups' centroid differ, with the Foreclosed group being larger, indicating more variation within this group. According to the group centroids, Foreclosed participants are more likely to perceive institutional support. Contrary to Foreclosed participants, those classified as Diffused, Moratorium, and Achieved are less likely to report Institutional Support. Separate discriminant analyses, using the items that assess support from each of the six assessed institutions, revealed that Religion was the institution that contributed to the reported support among participants from Nebraska, over and above the effect of the other institutions (i.e., the government, family, gender roles, political and legal institutions), $F(3,131)=5.33, \underline{p}<.01$.

A classification table was produced to assess the predictive accuracy of the function. As displayed in Table 18, the discriminant function predicting membership in the identity statuses correctly classified $49 \%$ of the cases, $x^{2}(3$, $\mathrm{N}=133)=12.53, \underline{\mathrm{p}}<.01$. The strongest prediction was to the Foreclosed group, 
with $85 \%$ of the participants accurately classified, compared to $0 \%$ to the Diffused and Moratorium, and only $48 \%$ to the Achieved group. In other words, the predictors are discriminating better between Foreclosed and Achieved group.

Brazil. The results for the sample from Brazil are presented in Tables 19 and 20. According to the stepwise discriminant analyses, two variables were sufficient to predict identity status in the World View domain in Brazil, namely, Gender and Institutional Change, $F(6,218)=4.58, p<.001$, with gender explaining $15 \%$ of variance alone. The rest of the variables washed out in the analysis. As can be seen in Table 20, the groups' centroid differ a great deal, with the Diffused group being larger in the first Function and the Moratorium group in the second function, indicating more variation within these groups.

The within-groups correlations are reported here because they show the relationships between the variables in the function -standardized (Klecka, 1980). These scores are then interpreted with the group centroid to determine their contributions to the discriminant functions (see Table 20). In function 1, NonCommitted participants are more likely than Committed participants to report Institutional Change depending on their gender. Non-Committed females (Diffused and Moratorium) are more likely than non-committed males to perceive institutional change. (Note: remember that gender was dummy coded as 1=males, 2=female). In contrast, Committed males are more likely to report Institutional Change than Committed females. In function 2, Explored participants are more likely to report Institutional Change than Non-Explored 
again depending on their gender. Explored males are more likely than Explored females to report Change. In contrast, Non-Explored females are more likely than Non-Explored males to report Institutional Change.

A separate stepwise discriminant analysis, using the items that assess institutional change in each of the six assessed institutions, revealed The Family as the social institution contributing to the reported overall institutional change in Brazil, over and above the effect of the other social institutions (i.e., the government, gender roles, religious, political and legal institutions), $F(3,110)=$ $4.49, \underline{p}<.01$, which may explain why perceived overall institutional change is mediated by one's gender in Brazil.

A classification table was produced to assess the predictive accuracy of the functions. As displayed in Table 19, the discriminant functions predicting membership in the identity statuses correctly classified $45 \%$ of the cases, $x^{2}(6$, $\mathrm{N}=114)=26.11, \underline{\mathrm{p}}<.001$. The strongest prediction was to the Achieved group, with $75 \%$ of the participants accurately classified, compared to $0 \%$ to the Moratorium, $25 \%$ to the Diffused, and $34 \%$ to the Foreclosed group. In other words, the predictors are discriminating better for the Foreclosed and Achieved group.

China. Table 21 lists some of the results from the discriminant analyses. Only one out of five discriminant variables (institutional change, openness, participation, accessibility, and support) was sufficient to account for the variance in the stepwise discriminat function, namely, Institutional Access, $F(3,51)=3.90$, 
$\underline{p}<.05$. The canonical correlation for the discriminant function was .432 , and by squaring the correlation it can be seen that $19 \%$ of the variance in the person's identity status can be explained by the model. (Note: Institutional Access is the average of perceived access across four instead of six social institutions in China: the family, gender roles, legal, and religious institutions. The government and political institutions were not allowed to be rated in China). As can be seen in Table 21, the groups' centroid differ a great deal, with the Foreclosed and Achieved group being larger, indicating more variation within these two groups. According to the stepwise discriminant analysis, thus, the variable that best predicted identity status in the World View domain in China is Institutional Access. Group centroids (Table 21) indicated that Foreclosed and Achieved participants (representing the Committed status) are more likely to perceive institutional access than moratorium (a non-committed status). In contrast to Foreclosed and Achieved, Diffused participants are less likely to report Institutional Access. A separate discriminant analyses, using the items that assess access to each of the four assessed institutions, revealed that Legal/Judicial institutions were the institutions that most contributed to the reported Institutional Access in China, over and above the effect of the other institutions (i.e., the family, gender roles, and religious institutions), $F(3,51)=$ $5.39, \mathrm{p}<.01$.

A classification table was produced to assess the predictive accuracy of the function. As displayed in Table 21, the discriminant function predicting 
membership in the identity statuses correctly classified $47 \%$ of the cases, $x^{2}(3$, $N=55)=10.63, p<.05$. The strongest prediction was to the Diffused group, with $85 \%$ of the participants accurately classified, compared to $0 \%$ to the Achieved group and only $5 \%$ and $43 \%$ to the Moratorium and Foreclosed group respectively.

Hypothesis 3 that stated that Institutional Attributes were predictive of Identity Status in the three identity domains was partially confirmed but only with the World View Identity Status and only within Nebraska, Brazil and China. Stepwise discriminant analyses showed that Identity Status in the World View domain was predicted by Institutional Support in Nebraska, Gender and Institutional Change in Brazil, and Institutional Access in China.

Hypothesis 4: Social Institutional change, openness, participation, accessibility, and support as indexed by the Attribution Scale of the SIR are predictive of Identity Distress/Satisfaction.

Because of the continuous nature of the Overall Attribution Scores of the SIR and the Satisfaction/Distress Scores of the IDS, multivariate regression analyses were used to investigate the predictive ability of the Institutional Attributes on Identity Satisfaction.

Before conducting multivariate regression tests, however, analyses were performed to establish whether a relationship existed between the demographic variables and Overall Identity Satisfaction (the added and averaged satisfaction across the three identity domains) for each site. These analyses were 
conducted in an effort to further statistically control for any influence the demographic variables may have on identity Satisfaction. In other words, if any of the demographic variables were related to Identity Satisfaction, the related demographics would have been entered in the regression as predictor(s) together with the institutional attributes. Institutional attributes (access, change, openness, participation, and support) were the only variables entered in the regression because none of the demographics were related to Total Identity Satisfaction in any of the sites.

Table 22 lists the results of the multivariate regressions by site. As it can be noted, some of the Institutional Attributes significantly predicted Identity Satisfaction in all sites, except in Sweden, $F(5,79)=.660, p=.65$. The remaining significant results thus refer to Brazil, China, Costa Rica, Miami and Nebraska. The variance explained by the institutional attributes on identity satisfaction ranged from $11 \%$ (in Miami) to $24 \%$ (in China). The Institutional Attribute that consistently predicted Identity Satisfaction across sites was Institutional Support, with standardized regression coefficients (betas) ranging from $b=.17$ (in Brazil) to $b=.33$ (in China). Notice that all betas were positive (Table 22) indicating that the more support they perceived from their social institutions, the more satisfied they were with their overall sense of identity.

Other institutional attributes showed the expected cross-cultural variability in predicting identity satisfaction depending on the particular site. Specifically, Institutional Accessibility significantly predicted Identity Satisfaction in Miami $(b=$ 
$.17)$, Brazil $(b=.20)$ and Costa Rica $(b=.34)$ but not in Nebraska nor in China. Institutional Openness to Change was a significant predictor of identity satisfaction in Nebraska $(b=.26)$ but not in the rest of the sites.

An interesting pattern emerged with Institutional Change when sites from Western Developed sites (Nebraska and Miami) are compared to Western Developing sites (Costa Rica and Brazil) and the Non-Western site (China). Institutional Change was significantly predictive of identity satisfaction only in China $(b=.24)$. Notice that the sign of the beta is positive (Table 22), indicating that the more Institutional Change is reported the higher is their identity satisfaction. Institutional Change was not predictive of Identity Satisfaction in Miami nor in Nebraska not even at the marginally significant level (when $p<.10$ ). Institutional Change, however, was marginally predictive of Identity Satisfaction in Costa Rica, $(b=.18)$ and in Brazil $(-.17)$ even though in contrasting directions. Notice again the opposite signs in the betas in the Western developing countries (Table 22) meaning that in Costa Rica, more Institutional Change was marginally predictive of increased Identity Satisfaction, $t(112)=1.71, p=.091$. In Brazil, however, more Institutional Change marginally predicted less Identity Satisfaction, $\mathrm{t}(153)=-1.94, \mathrm{p}=.055$. Finally, Participation in the reported institutional changes was not predictive of Identity Satisfaction in any of the sites. Nevertheless, Participation in the institutional changes predicted Identity Distress in Nebraska $(b=-.24)$, Brazil $(b=-.27)$, and Costa Rica $(b=-.25)$. Notice the negative betas which indicate that the more Identity Distress is reported by 
participants in Nebraska, Brazil, and Costa Rica, the less Participation in the changes taking place in their social institutions was reported. In other words, disengagement from the changes taking place in their social institutions predict higher Identity Distress.

Hypothesis 4 which stated that Identity Satisfaction would be predicted by Social Institutional Attributes was confirmed, except in Sweden. Institutional Support consistently predicted Overall Identity Satisfaction in Brazil, China, Costa Rica, Miami, and Nebraska. Institutional Access predicted Identity Satisfaction in Costa Rica and in Miami. In Nebraska, Identity Satisfaction was predicted by Institutional Openness to Change. Lastly, Institutional Change predicted Identity Satisfaction in China and marginally predicted Satisfaction in Costa Rica and in Brazil. In Brazil, however, more change was marginally predictive of lowered Identity Satisfaction. Participation in the reported Institutional Changes did not predict Identity Satisfaction in any site. Nonetheless, it predicted Identity Distress in Nebraska, Brazil, and Costa Rica, with less participation predicting more distress.

Discussion and Summary

\section{Research question}

Although a literature that study identity formation during adolescence has considerably grown, it has been mainly derived from studies conducted within ethnic/culturally homogeneous samples, mostly from the U.S. This has limited the generalizabilty of the findings to adolescents of different linguistic and 
ethnic/cultural traditions living in varied sociocultural contexts. Furthermore, although research using the identity status paradigm has been conducted within a developmental framework that explicitly recognizes the role of social contexts in an individual's life including, for instance, the role of social institutions, little or no empirical work has been done to more closely examine this relationship.

To begin exploring the relationship of sociocultural factors and identity at the cross-cultural level, a study was conducted investigating the effects of variation between ethnic/cultural groups on identity status and satisfaction and the empirical relation of institutional variation and identity status and satisfaction within ethnic/cultural groups. Participants for this study included late adolescent college students from six sites representing different linguistic and ethnic/cultural groups from five countries (USA, Sweden, Costa Rica, China, and Brazil).

Summary of results

As hypothesized, differences were found among sites in the personal, interpersonal, and world view status. The distribution of the status among sites, however, was more complex than hypothesized. Similarly, the hypothesis predicting differences among sites in identity satisfaction/distress was confirmed. The hypothesis with regard to the direction of the differences, however, received mixed support. Another important finding was the partial confirmation of the hypothesized relationship between social institutions and identity status and satisfaction within each site except in Sweden. This relationship, however, was not as strong as expected. In addition, the relationship of sociodemographic 
variables and the identity status and satisfaction variables showed more patterns of cultural specificity rather than transcultural patterns of influence.

The overall findings of this study point to possible psychological, interpersonal, and institutional processes linking cultural differences, social institutions and identity formation. Furthermore, this study can shed some light on how cultural differences may be mediating the effect of the impact that sociocultural factors have on human development at the psychological level indexed by identity status and satisfaction.

\section{Integration of results with existing literature}

It has been argued that culture, in the form of standardized practices, communal attitudes, values and customs permeate the biological, social, and psychological components of an individual's growth and development (e.g., Macionistl, 1997; Nsamenang, 1992; Nugent, 1989; Stigller, Shweder, \& Herdt, 1990). Furthermore, the particular social institutions in a culture that represent formalized ways of assisting, regulating, and promoting human interaction can provide a cue as to the possible mechanisms by which society influences the individual.

A specific mechanism by which a society can encourage particular behaviors, values and attitudes is through parental practices (Hamner \& Turner, 1990; Harkness \& Super, Harwood, 1995; Lancy, 1996; Shwalb \& Shwalb, 1996). It has been documented that Asian cultures, for example, expect more conformity to parental views than do Western cultures (Poole, Cooney, \& 
Cheong, 1986). On the other hand, parental practices of encouraging problem solving and allowing adolescents to individuate have been linked to adolescents' identity formation and higher ego development in western societies (Cooper, Grotevant, \& Condon, 1984; Grotevant \& Cooper, 1985; Hauser, Powers, Noam, Jacobson, Weiss, \& Follansbee, 1984). Therefore, cultural differences in parental practices may be one of the mechanisms responsible for the strong cultural variation in identity status and satisfaction found in this study.

Although there is an absence of studies that empirically examine the relationship between social institutions and identity status, there is some evidence that in addition to family, other social institutions play a prominent role in adolescents' behavior and development, particularly schools (Cotterell, 1996; Hurrelmann, 1996) and the job market (Coté \& Allahar, 1996). Some specific mechanisms involved in the linkage between social institutions and adolescent's development have been elucidated. For instance, the quality of attachment to parents and to the school have been related to susceptibility to smoking and alcohol abuse, and proneness to engage in antisocial behaviors (see e.g., Dishion, Patterson, Stoolmiller, \& Skinner, 1991; Smith, Canter, \& Robin, 1989). Furthermore, specific qualities of the social institutions in which adolescents are embedded have been related to adolescents' well being. Support from school, for instance, have shown to be related to personal development during adolescence in many dimensions ranging from prevention of antisocial behavior and health impairment to facilitating more effective entrance 
into the job market (Hurrelman, 1996). A changing job market, at least in advanced industrial societies, has been identified as one of the most important factors contributing to identity difficulties faced by adolescents particularly when the linkage between higher education and the workplace is examined in the context of unemployment. Coté \& Allahar (1996), for example, have provided some evidence on the emotional cost on adolescents associated with current institutional changes statistically indexed by substantial increments in suicide rate, compromised psychological well-being, and high mortality and morbidity rates (Coté \& Allahar, 1996, pp. 48-65).

Other studies have shown the impact that economic hardship and unemployment have on human behavior and development. For instance, Peterson et al. (1996) has cited the seminal work of Jahoda, Lazarsfeld and Zeisel (1933) that emphasized the psychological significance of economic deprivation on the development of the young in an Australian village and the work of Elder and colleagues (e.g., Elder \& Caspi, 1988) that examined the impact of the Great Depression on U.S. adolescents' psychosocial development. Peterson et al. also cited the role of family processes in linking economic problems to maladjustment in West Germany (Silbereisen, Walper, \& Albrecht, 1990), and the negative effect of poverty on emotional tone or mode in some non-Western nations (Offer, Ostrov, Howard, \& Atkinson, 1988) in addition to poor families reporting more psychological problems (Venkata Rami Reddy, 1979), poorer health and performing worse on intelligence tests (Misra, 1982). 
The results of the present study also revealed that social institutions are related to identity status and satisfaction. The results of this study, however, showed marked cultural specific patterns of relation rather than transcultural patterns of influence.

Interpretation of results.

China. One important finding in this study was the cultural variation observed in significant differences in the distribution of identity statuses between sites in the three identity domains. Furthermore, no consistent patterns of status distribution across all three domains were evident for any particular site, except for China. In China, two non-committed statuses were present more often than expected, viz., the diffused and the moratorium status.

A more detailed examination of this finding suggests that perhaps ideology in the sense of beliefs about the world that make up the individual's world view, are influenced by political ideology. In this context, it is interesting to note that the diffused status was present more often than expected and consistently across the three identity domains (personal, interpersonal and world view) but with "diffused" in the "world view" being the highest of three domains. Indeed, in China, diffused world view occurred more frequently than in any other site.

It is also in this context interesting to note that, in China, it was within the world view domain that institutional access predicted identity status but not in any other domain. Moreover, an examination of the direction of the results 
indicated that students in the diffused status reported significantly lower institutional access than students in the moratorium status. The fact that exploration in world view (i.e., moratorium) was related to institutional accessibility only in China, a country with a political system that has not typically encouraged its citizens to question and openly challenge its own institutions, supports the view of the possible relationship between institutionalized political ideology and identity formation in the world view domain. That is, institutional efforts to make institutions inaccessible to question and challenge may translate into an uninvolved (diffused) attitude towards issues of beliefs about the world view at the psychological level among some college students.

It is highly revealing that in this study Chinese participants were not allowed to evaluate some of the most influential institutions in China, viz., the government and political institutions. One can only wonder how having an institutionalized way to curtail people's free expression in China may foster a diffused stance towards their personal identity. A political and military machinery that has openly and strongly discouraged any attempts to question the prevailing communist apparatus in China may have also contributed to institutionalize more conforming attitudes among some of its citizens aided by a culturally prescribed view to encourage children's conformity to the established. In contrast, some other individuals may view enough changing conditions in their surrounding social institutions as to be less conforming but rather to venture in 
exploratory behaviors indexed by the high proportion of moratorium status in China compared to the other sites.

Some institutions in China have been described by some authors to be experiencing "profound" although strictly monitored changes, particularly seen in the movement from a totally controlled to a market economy accompanied by extremely rapid economic growth (Goodman, 1984; Unger \& McCormick, 1996; Yunling, 1995). Perhaps, this "opening up" to the outside world has been significantly enough as to encourage some young people to actively explore the options recently available in their society, for instance, in the form of varied occupational opportunities and new forms of political economies.

Although a market economy in China has created, for instance, increasing materialistic expectations in their citizens and belief in their capacity to acquire material goods (Anonymous, 1996), China is also experiencing the restructuring of the job market and raising unemployment which may make the process of reaching a commitment with regard to occupational choices a very difficult task in China. One could speculate that China's "profound" and "positive changes" in some of its social institutions (Goodman, 1994) may have had a psychological costs among college students.

Although institutional change was positively related to identity satisfaction in China, the finding that Chinese participants were consistently classified more often than expected in the diffused status is provocative. Furthermore, Chinese participants reported the lowest identity satisfaction across the three identity 
domains compared to the rest of the sites. This finding appears to be suggestive with respect to the issue of the psychological adaptiveness of the identity statuses such as the Diffused and Moratorium or any other status for that matter.

Because of the significant proportion of diffused participants in the three identity domains compared to the rest of the sites, it was possible, within the constraint of this data, to begin to explore this question in the Chinese sample, by examining the relationship between the Diffused status and the available indices of adjustment, viz., identity satisfaction and distress. The results of three separate one way ANOVAs using identity status as the independent variable and identity satisfaction/distress as the dependant variable showed that in the Chinese sample identity satisfaction/distress does not depend on identity status neither in the personal, $\mathrm{F}(4,63)=1.68$, $\mathrm{p}=.17$, interpersonal, $\mathrm{F}(4,49)=1.27, \mathrm{p}=$ .29 , nor in the world view domain, $F(3,51)=.31, p=.82$.

While not definitive, the results indicate that there was little effect of identity status on adjustment in the Chinese sample. These results thus speak to the issue of the need for caution when making transcultural generalizations based on a culturally homogeneous data base. Furthermore, these results speak to the issue of being more than careful when making universal claims to identity formation based on a culture bound conceptualization of human development. It is unknown whether identity as it has been described in the psychological literature has the same developmental trajectory, psychological function and meaning for adolescents in cultures different than ours. 
An alternative explanation to the findings in China, or in any of the remaining sites for that matter, is the possibility that the differences found among sites in identity status and satisfaction is just the product of assigning different meanings to the same questions asked in the survey. To what extent answering "yes" or "no" to an item that taps into the dimensions of exploration and commitment has the same meaning in different sociocultural contexts is an issue that cannot be addressed with this type of data.

Sweden. A second unexpected finding was the high proportion of the Diffused status in the personal and interpersonal domains and Foreclosure in the personal and world view domain in Sweden, both statuses representing the nonexplored dimension of the identity status paradigm. This finding is perplexing in light of the long-standing democratic tradition of the Swedish society that has valued independent thinking and exploration of alternatives in the context of relatively non-drastic changes in their social institutions that so far have been very supportive of their youth. Indeed, some authors have used Sweden as a model to imitate to counteract the "disenfranchisement" of youth from their social institutions (Coté and Allahar, 1996).

Citing statistical data from Sweden, these authors have argued how a "compasionate" society tried to reverse the raising rate of unemployment for youth aged 16 to 24 during the world wide recession of the early 1980 s and 1990s, a decline in earning power, and changes in educational policy greatly affecting the youth in particular (Coté \& Allahar, 1996). (Note: Sweden's rate of 
unemployment is very low even compared to the U.S. and much less to Latin American developing countries. Nonetheless, as noted by Coté and Allahar, even small increases of unemployment are cause for great concern among Swedish given its government commitment to full employment).

The Swedish government appropriately responded to unemployment threats to its youth by creating social policies that included free educational tuition and generous loan and grant system, job counseling, training and placement, and a child grant allowance that transfers to a student grant when the child becomes 16 that in turns is directly received by the recipient between the ages of 18 and 20 (Coté \& Allahar, 1996). Incidentally, Sweden spends $7.4 \%$ of its gross domestic product (GDP) on education, one of the highest in the world (Roe, Bjurström, \& Fornäs, 1994).

One can only conjecture of the effect of having such a responsive society that can even anticipate to its youth's needs in such an effective way as evidenced by almost full employment among today's young Swedes, high involvement in sociocultural activities, a decline in drug use, and a socially conscious generation (Coté \& Allahar, 1996, pp. 151-158). To what extent a society that is so generous with its youth, that provides ample access to its institutions, and provide extensive institutional guidance and support to its members would encourage people to explore more options on their own.

Perhaps Sweden's highly protective socioeconomic context does not promote exploration of available options among its youth but fosters extensive 
dependency and guidance from adults that is reflected in the high proportion of two non-explored statuses (diffused and foreclosed) compared to the rest of the sites. One evidence of the increasing young Swedes' reliance on adults is the high number of late adolescents who live with their parents. Almost $50 \%$ of males aged 20 to 24 lived with their parents in 1985 (Coté \& Allahar, 1996, p. 154). Indeed, Roe et al. (1994) have expressed the concern that in Sweden the "adult intervention in the lives of adolescents has become too extensive" (p. 383) by limiting the youth's opportunities to develop an identity on their own. To what extent being achieved is more adaptive than being foreclosed in populations other than White North Americans is an issue open for future research.

\section{Commonality of results between China and Sweden. An intriguing} commonality between China and Sweden in this study was the lower identity satisfaction across the three identity domains (personal, interpersonal and world view) in both countries compared to the remaining sites (Costa Rica, Brazil, Miami and Nebraska). In fact, participants from China and Sweden reported the lowest identity satisfaction across domains of all sites. Even though China and Sweden belong to different geographic regions and different political systems one western and democratic and the other one a non-western communist country- the economic systems and institutions of both societies, nonetheless, more closely approximate a socialist model than, for instance, the U.S., Costa Rica and Brazil, which tend to be based on a more capitalist model. In this context, the results appear to suggest that members of more socialized 
economic and other institutional systems (e.g., health care, education, welfare) will tend to report less identity satisfaction compared to members of more capitalist systems.

Less identity satisfaction among members of socialist countries compared to more capitalist ones could be due to the high value that socialist systems place on the group or collective good rather than the high value of the individual characteristic of capitalist systems. The finding that participants in China and Sweden reported lower identity satisfaction than participants in the U.S., Brazil, and China may not be perplexing to the extent that capitalism allows individuals to self-direct their lives (see e.g., Sennet, 1997) (more so than socialist systems), and to the extent that directing one's life more actively is reflected on the satisfaction with one's identity issues. While suggestive, these findings of course are not definitive.

An alternative explanation is that the differences found among sites is due to culturally determined influences in the mode of expression. Some ethnic cultural groups have certain communicative styles that when describing an event appear to use verbal expressions that seem a bit exaggerated to an outsider. The "choteo" among Cubans, for instance, involves self-criticism and exaggeration of things out of proportion together with a rapid rate and high volume of speech (Bernal, 1982). This behavior could somehow be transferred to selecting descriptive words used in surveys such as "very much", "extremely satisfied", etc., that might later result in inflated findings. Indeed, some studies 
have found differences in modes of expressing distress even within an ethnic group as a function of acculturation (Haberman, 1976). To what extent differences in the mode of expression among participants from Sweden and China compared to Costa Rica, Brazil, and Hispanics and Anglos from the U.S. account for differences found in this study is a question that can be addressed in other studies.

Brazil and Costa Rica. The hypothesized distribution of identity statuses in the two Latin American countries sampled in this study was generally confirmed. Participants from Costa Rica were classified in a non-explored status (Diffused) more often than expected in both the interpersonal and world view domains. In addition, there were more Diffused status found in Brazil in the personal domain and more Foreclosed in the interpersonal domain than expected, both statuses representing the non-explored dimension of identity formation. Brazil and Costa Rica are two developing countries confronted with formidable challenges ranging from drastic welfare reforms in Costa Rica (MesaLago, 1997) to an astonishing rise in consumer prices of more than 900 percent in less than one year in Brazil (Interamerican Development Bank, 1989, p. 285) added to unplanned rates of high urbanization processes as result of external and internal immigration in both countries.

The living conditions of a large percentage of young people in Brazil, for example, may not even allow the youth to have time to engage in the search and exploratory process during adolescence argued to be essential for identity 
development. Data from the UNICEF in 1989 showed that almost $30 \%$ of those between the ages of 10 to 17 are engaged in laboring activities, and of these, $64 \%$ work more than 40 hours a week (Pereira \& Heringer, 1994).

Costa Ricans are also experiencing a rise in unemployment as well as underemployment (Gindling, 1993) in addition to high urbanization rates due to massive immigration from neighboring countries in military and political turmoil (Nicaragua and Panama) that have promoted the deterioration of the living conditions of the majority of the population. These years have been markedly difficult for the fluctuating economy in both countries that have accentuated one of the most pressing problems faced by Latin American societies: excessively marked social inequalities that selectively limit institutional access and resources to the most needed. Indeed, these years of extensive institutional change have been described by some authors as a "predatory and disorderly type of growth" (Pereira \& Heringer, 1994, p. 5).

It is illustrative that institutional access was significantly associated with total identity satisfaction in a positive direction in both, Costa Rica and Brazil. This means that those who perceived more institutional access reported higher identity satisfaction. Having scarce financial resources can limit one's opportunities to experiment with available roles, goals, and values in a particular context. Derived from statistical data from North America, Coté and Allahar (1996) have provided convincing evidence of the clear limitations to the opportunities that society afford to some segments of the population, particularly 
to racial minority and lower class youths, as indexed by higher school drop out and lower retention rate at high school and college levels, higher unemployment and poverty rate, and the most severe earning decline compared to peers with more resources (Coté \& Allahar, 1996). It is not hard to imagine that under these conditions the process of identity exploration for the youth in these countries appear thwarted by lack of substantive resources to afford, for instance, an extended period of psychosocial moratorium.

The moderating effect of having a newly emerging democracy that is attempting to create more accessibility to its limited, but nevertheless more available, resources than in previous years (Pereira \& Heringer, 1994) may be reflected in the finding that although there were more non-explored statuses in Brazil in the personal and interpersonal domains, the identity satisfaction of Brazilian participants was relatively high. In fact, the identity satisfaction of participants from Brazil in both the interpersonal and world view domains was the highest among all sites in spite of the fact that in Brazil institutional change was negatively related to identity satisfaction.

As it was noticed earlier, Brazil has experienced significant institutional transitions (sprinkled with bloody confrontations between civilians and the police) that have taken place in the context of change from a military government to a path of controlled political opening during the early seventies to more democratic forms of life in the eighties (Cavarozzi, 1992) that have extended to the present. Furthermore, institutional changes in Brazil are evidenced for example by an 
extended controversial legislation that resulted in a new constitution and contained numerous complex provisions including The Children and Adolescent's Act of 1990 that introduced new possibilities for Brazilian's youth (Pereira \& Heringer, 1994). Not surprisingly, change was the institutional attribute that best discriminated among the identity statuses in the world view domain in Brazil, with participants classified in the non-committed statuses (diffused and moratorium) perceiving more change than those in the committed statuses (foreclosed and achieved).

A paradoxical finding in Costa Rica was the high proportion of Achieved status in both the personal and interpersonal domain, compared to the rest of the sites. Although this finding could have been the result of having an older sample in Costa Rica, age was not significantly related to identity status either in the interpersonal domain nor in the world view across sites. Identity status in the personal domain, however, was positively related to age. Given that an achieved status is found more commonly among older adolescents (Benson et al., 1992; Christopherson et al., 1988; Prager, 1986) and in longitudinal studies the number of identity achievers increases over time (Adams \& Fitch, 1982; Fitch \& Adams, 1983; Kroger \& Haslett, 1988; Waterman et al., 1974; Waterman \& Goldman, 1976), age was statistically controlled in the analyses when comparing the status distribution among sites. Differences in identity statuses among sites still persisted after controlling for age in the personal domain. 
A possible explanation for the high proportion of Achieved participants in Costa Rica may be the lack of equivalence of the sampled populations. The sample from Costa Rica was drawn from a small private university in contrast to the remaining samples that were drawn from large-sized public universities. The high proportion of identity achievers may be due to a selective population in the Costa Rican sample to the extent that a sizeable proportion of the students in a private university come from a distinct socioeconomic background belonging to an elite in their own context rather than representing the general college population. This is assuming, however, that socioeconomic status is related to identity status.

When the subjective rating of participants' own SES is compared among sites, participants from Costa Rica reported one of the lowest SES mean of all sites together with China. Within the Costa Rican sample, however, SES was positively related to identity status in both the personal and the world view domains, indicating that higher SES was associated with the explored statuses (moratorium and achieved) and lower SES was associated with the non-explored statuses (diffused and foreclosed). Having a sample that includes subgroups with sharp contrasting SES within site may have accounted for the simultaneous high proportion of diffused and achieved status in Costa Rica compared to the rest of the sites. To the extent that availability of socioeconomic resources facilitates personal growth (McClain, 1975; Coté \& Allahar, 1996) as well as 
identity satisfaction also in Costa Rica, the alternative explanation of participants from Costa Rica being a selected sample cannot be ruled out.

In addition to having more participants classified as Achieved in the personal and interpersonal domain, participants from Costa Rica also reported the highest identity satisfaction of all sites in the personal domain that was significantly different from the satisfaction reported by participants from Nebraska, Sweden, and China. This could have been the result of having more identity achievers in the personal domain in Costa Rica compared to the rest of the sites. Alternatively, these differences could have also been due to cultural differences in modes of expression as previously stated. Incidentally, the highest satisfaction in the personal domain was reported by participants of Latin American background (Costa Rica, Brazil, and Hispanics from the U.S.).

Miami and Nebraska. As hypothesized, participants from the U.S. were classified in the explored statuses more often than expected. Specifically, there was a high proportion of moratorium status in the personal domain in Nebraska, and of Achieved status in Miami, in both the interpersonal and world view domains. In addition, participants from Nebraska and Miami reported one of the highest identity satisfaction in both the interpersonal and world view domains compared to the rest of the sites.

The fact that Hispanics from Miami were classified more often than expected in an Achieved status may surprise a reader who is acquainted with other studies in the United States that have shown that other ethnic minorities 
tend to be more foreclosed rather than achieved when compared to nonminority samples (e.g., Abraham, 1986; Hauser, 1972; Streimatter, 1988). The Hispanic sample used in this study, however, represented a majority of students of Cuban American background as evidenced by the population from which this sample was drawn, viz., Miami. Cuban Americans when compared to the rest of Hispanic groups in the United States have the highest educational level, the highest median income, the lowest unemployment rate, and the lowest incidence of poverty at least compared to Hispanics of Mexican and Puerto Rican background (U.S. Census of the Bureau, 1993).

These factors combined with the fact that Cuban Americans are a nonminority group in the local context may produce a qualitatively different subgroup of Hispanics that did not differ from Whites from Nebraska on the variables of interest in this study. Not surprisingly, perceived institutional support by participants from the U.S. was significantly associated with total identity satisfaction in both Nebraska and Miami. Furthermore, the size of the betas in the regression analyses were almost the same in both samples.

The finding that participants from Nebraska were classified in the moratorium status more often than expected compared to the rest of the sites, confirmed one of the hypothesized differences in identity statuses among sites. It also provides some evidence for Coté and Allahar's (1996) argument that one of the consequences of the changes taking place in advanced industrialized societies is that youth in such societies are experiencing a longer period of 
psychosocial moratoria, particularly among those who attend college.

Nevertheless, it is not known from this data how long these young people have been or will be in this status. Longitudinal studies with college samples in North America, however, have shown that moratorium is not a stable status and that most youth commit themselves to a form of action (e.g., Adams \& Fitch, 1982; Fitch \& Adams, 1983; Waterman et al., 1974; Waterman \& Goldman, 1976), at least among those who can financially and other wise afford this moratorium and its subsequent commitment.

Transcultural versus culture specific sociocultural factors. The overall findings of this study also help to shed light on the issue of the role of transcultural versus culture specific sociocultural factors in identity development. One important finding with respect to this issue was the striking pattern of cultural specificity in the relationships between the sociodemographic variables and the identity status and satisfaction variables. Sociodemographic variables, for example, were related to identity status in every country. There was, however, no consistent pattern of relationships. Gender, for example, was significantly related to identity status in only one of the six sites, namely, in Brazil.

The effects for SES, age, and grade showed a similar pattern of cultural specificity with very little overlap between sites.

A second important finding with respect to the issue of transcultural versus cultural specific was the similar marked pattern of cultural specificity in the relationships between the sociodemographic variables and the identity 
satisfaction variables. Sociodemographic variables, for example, were related to identity satisfaction in every country. Once again, however, there was no consistent pattern of relationships. Gender, for example, was significantly related to identity satisfaction in only one of the five countries, namely, the US (both in Miami and Nebraska). The effects for SES, age, and grade showed a similar pattern of cultural specificity with very little overlap between countries. The culture specific effect of sociodemographic indices on identity status and satisfaction illustrate how sociodemographic variables operate differently depending on the context in which these variables interact.

A third important finding with respect to the issue of transcultural versus cultural specificity was the similar pattern of cultural specificity in the relationships between the social institutional attributes and the identity statuses. The pattern of institutional attributes that were predictive of identity status was again strikingly culture specific. There were few institutional attribute variables, for example, that predicted identity status within countries, and those that were predictive were entirely different for each country. Institutional Support, for example, significantly predicted world view identity status in Nebraska, Institutional Change in Brazil, and Institutional Access in China. Even the specific social institutions that contributed most to predict identity status in the world view domain were different in every country. Religion, for example, predicted identity status in Nebraska, the Family in Brazil, and Legal Institutions 
in China. Thus, there was no institutional attributes nor a specific social institution that consistently predicted status across countries.

The pattern of the institutional attributes associated with identity satisfaction tended again to be culture specific. There were a number of institutional attribute variables, for example, that were associated with identity satisfaction varying for each country. One institutional attribute, however, displayed more transcultural consistency. Specifically, institutional support was a significant predictor of identity satisfaction in five sites (China, Costa Rica, Brazil, Miami and Nebraska). This finding points to a more transcultural pattern of the alluded positive effect of institutional support .

Other institutional attribute variables (i.e., change, openness to change and access) showed more culture specific patterns of influence on identity satisfaction. For instance, institutional change predicted satisfaction in China, Brazil and Costa Rica (albeit at the marginally significant level in the last two countries) but in different directions. Institutional change, for example, positively predicted identity satisfaction in China and Costa Rica. In Brazil, however, more institutional change was associated with lowered levels of identity satisfaction. Finally, institutional openness to change predicted identity satisfaction in Nebraska only.

This differential effect of institutional change on identity satisfaction depending on the particular society where is occurring not only attests for the hypothesized influence of social institutions on identity formation in the 
hypothesized most changing societies sampled in this study (Brazil, China, and Costa Rica) but also how one specific social process (i.e., social change) can be positive and desirable in one context but not in another. Parenthetically, changes in China have been evaluated positively in the socio-economic and political literature which was also perceived positively at the psychological level by participants in China in contrast to Brazil. More perceived changes in Brazil were associated with decreased identity satisfaction. This finding also illustrates the need to not only contextualize our findings but to use modesty when addressing the implications derived from cross-cultural research to avoid faulty generalizations.

\section{$\underline{\text { Implications }}$}

Some of the implications of this study clearly illustrate the imperative need to assess development, broadly defined, as a culturally adaptive phenomenon (Berry, 1981) that necessarily must include an assessment of the behavior in context (Sameroff, 1986) as well as at different systemic levels (Bronfenbrenner, 1986). This has not been the common practice in the psychological literature in general and much less on the identity status literature. The few world-wide and cross-cultural studies that have studied identity formation using the identity status paradigm have not included measures that index psychological adjustment, and thus, have been subjected to questionable generalization about the function and psychological adaptiveness of identity status in different contexts. The findings of this study call for the need to 
empirically validate our theoretical constructs before continuing to apply them to different populations.

With regard to the relationship of social institutions and adolescents' development, these findings also call to the need to further study this issue in context. The transcultural pattern of relation between institutional support and identity satisfaction, however, is highly telling of the mediating role that social institutions can have on young people's life to counteract somehow the suggested normative psychological distress that accompany the task of forming an identity. In the context of school as a social institution, some authors have proposed strengthening mentoring relationships as a particular intervention to assist youth in the school setting. By strengthening mentoring relationships the youth can benefit from adult mentors who promote competence and provide selfassurance in the face of new situations (Hamilton \& Darling, 1996).

The example of the protective policies introduced in Sweden to counteract unemployment and decreasing earning power among its youth is another convincing argument that responsive and supportive social institutions can make a positive difference in young people's life (Coté \& Allahar, 1996). To what extent other societies with less resources than Sweden can do this type of intervention is a practical limitation that concerned social policy makers will have to take into account. In addition, it is unknown whether this type of intervention would prove effective in a context other than Sweden, but it clearly illustrates the need to assist the youth as much as possible at an institutional level. 
A problem in contemporary society is that many times the social institutions that are suppose to guide and support the youth during this developmental transition are experiencing disarray in themselves evidenced by the continued restructuring in societies' primary institutions in addition to confusing and contradictory values in prevailing ideologies. Not surprisingly, some young people find themselves experiencing increased psychological distress as well as disenfranchisement from their institutions. This was evidenced in further analyses of the data (not shown here), for instance, by the negative association between participation in institutional change and identity distress in Nebraska, Brazil, and Costa Rica, indicating that less participation in the changes of social institutions was associated with more identity distress. As in any other research, however, these implications are bounded to the limitations of this study.

\section{Limitations}

As in most cross-cultural research, the most pressing limitation of this data is the issue of cross cultural equivalence in the instruments used. In this study, double successive translations and factorially derived scores were used to approximate cross-culturally equivalent instruments. Although successive double translation (Brislin, 1986) increases culturally equivalence in the instruments, it does not guarantee that the constructs used have the same meaning across cultures. Furthermore, even a factor analysis that confirms a similar factor structure in an instrument has been criticized by authors who have 
suggested more recent mathematical models to test for cultural equivalence in the measures used to do comparative studies.

Van de Vijver and Poortinga (1991), for example, have proposed a conceptual framework for the analysis of scores based on generalizability theory that requires more than a simple inspection of item bias statistics used with raw data (analysis of variance and item response models) and aggregated data (such as the use of factor analysis, comparison of correlation matrices, analysis of $p$-values, transformed item difficulties, linear structural models, and chi square approaches). Their proposed analysis sum to a multiple regression model in which the statistical technique used is a hierarchical regression analysis. For the mathematical rationale and specific examples of the proposed model, the interested reader is referred to Van de Vijver and Poortinga (1991).

Another limitation in this study was the low internal consistency of two scales of the IDS in some sites. The lowest reliability was shown in the world view scale, particularly in Sweden $(\alpha=.03)$ and Brazil $(\alpha=.37)$. When a measure has low internal consistency it is difficult to tell whether some of the differences found represent real differences or they are simply the product of an unreliable scale. Fortunately, the rest of the scales in both the IDS and SIR showed modest to high internal consistency in all sites.

The discriminant ability of the institutional scores on identity status in the three domains, however, was rather weak. This may have been due to the scoring procedure used in this study. The issue, then, becomes methodological 
rather than theoretical. The score used to index the institutional attributes scores were the most global scores that can be used with the SIR. The institutional attribute scores consist of the average response to the rating of all the social institutions added together and thus represent a global evaluation of social institutions that might no be sensitive enough to detect enough variance as to differentiate among the three domains of identity development. Additionally, if there is a stronger discriminatory ability of the institutional attributes on identity status, the effects may be "washed out" when the rating of several institutions are pooled together. A different scoring procedure can be tried out in further research whereby the institutional attributes and evaluation of each social institution is scored separately in order to test the relationship of each social institution with identity status or with identity satisfaction for that matter.

The scoring procedure of the SIR may also have a bearing when interpreting the statistically significant but rather weak association between institutional attributes and identity satisfaction. The explained variance of the association of institutional attributes and identity satisfaction ranged from 11 to $24 \%$ only. Individual institutions may have a stronger relation with some of the three identity domains rather than all the institutions pooled together in one score. It is not difficult to conceptualize, for instance, that political and religious institutions may have a stronger association with the world view domain (moral, political, and religious issues) rather than, say, with the personal domain (career 
goals, long-term goals, sense of self). Perhaps the family and educational institutions have a stronger association with the later instead.

Another reason for not having found a stronger association between social institutions and identity satisfaction could have been due to the relatively similar identity satisfaction reported by participants within each site in addition to the previously stated explanation of the global nature of the institutional attributes score. College students might be generally satisfied with their sense of identity across sites and, therefore, the explained variance of the satisfaction score in the regression analyses cannot be that large. After all, college students are a more selected sample than the general population. A larger effect size may be found in samples from the population of late adolescents other than college students, which brings me to address the limitations of the study with regard to generalizability issues.

The generalizability of the findings to young people other than college students is a limitation that not only applies to this study but to an overwhelming majority of research in the identity literature or in Psychology for that matter. Most psychological studies have been conducted exclusively with college participants. Using college students as participants in our studies is not a problem in and of itself. The problem is the unjustifiable overgeneralizations made from culturally bounded samples and constructs. Some psychological accounts and even major theories of human development have been offered as explanations to universally present human phenomenon to the exclusion of the 
contexts in which human behavior and development takes place. At best such a phenomenon might be present only in that particular population from which the theory was derived. The findings of this study, thus, are at the least applicable to college students in these sites and at the most to college populations in general.

A final possible limitation in this study with regard to sample selection is the questionable equivalence of the Costa Rican sample with the rest of the sites. Participants from Costa Rica were recruited at a small private university compared to large-sized public universities in the rest of the sites. Van de Vijver and Poortinga (1991) have pointed out that cultures differ in many more ways than what it is usually assumed and have strongly recommended to include when possible the assessment of other background variables such as education, socioeconomic status, and other variables that may act as confounding that affect the variable(s) of interest.

It has to be highlighted that this study represents a great improvement in the identity literature at the methodological level by having assessed five sociodemographic variables in all six sites. These variables were then able to be statistically controlled for their possible effect on the variable of interests that, as it has been stressed throughout this section, showed more cultural than transcultural specificity.

Suggestions for future research

Perhaps the most important finding of the study, then, speaks to the issue of transcultural versus culture specific influences of sociocultural factors on 
identity development. The study was conducted within a co-constructivist framework that, like other approaches, views identity formation as a psychosocial developmental process. That is, a developmental process that is influenced by both psychological and social processes. To this, the co-constructivist approach adds the view that the organization of the social processes that have an impact on identity formation have a complex dimensionality of structure which include both transcultural and culture specific components.

Within the limits of the scope of this study the overall pattern of results provided considerable evidence for the cultural specificity of the influence of culture but little for the transcultural influences of sociocultural factors. From a co-constructivist perspective, these findings appear to not only speak to the issues related to developmental theory, but also to the broader issues of the cultural expression of our age. More specifically, the emergence of an increasingly articulate postmodern discourse has raised challenges with respect to the importance and significance of universal and transcultural process to human development (Chandler, 1995). The emerging postmodern tradition, in contrast to the modern tradition, emphasizes knowledge that is local and particular rather than universal and cumulated (Lyotard, 1979). In other words, the postmodernist tradition explicitly recognizes the contingent and contextual nature of knowledge, and the impossibility of any mode of human inquiry (including science and philosophy) to transcend the boundaries of its cultural and historical horizon (Kvale, 1992; Lyotard, 1979; Rorty, 1992). 
The results of this study, though limited in scope, appear to support the value of knowledge that is local and particular in nature. More importantly, it clearly call attention to the need for future research exploring in a systematic way the relative importance of transcultural versus culture specific components of identity and its psychological function in varied sociocultural contexts. In addition, these results call attention to the need of empirically examining more closely the relationship between variables at the macrosystemic level (i.e., cultural variation and social institutions) and variables at the microsystemic level (i.e., identity status and identity satisfaction) also in varied sociocultural contexts.

A broader goal in this dissertation was to begin exploring how sociocultural factors in a particular context have an impact on individuals' identity development as indexed by their identity status and satisfaction. A direct answer to this question cannot be made with the type of data collected. However, drawing from different disciplines that are concerned with factors that affect adolescent's life I have attempted to begin examining possible linkages between society and the individual at the psychological level. Future studies can investigate variables at the macrosystemic level (cultural variation and social institutions) and their relationship with variables at the microsystemic level (identity status and many other psychological variables). In addition, comparative work that examines this relationship in varied social contexts, particularly in developing and non-western countries is sorely needed. 
This type of research is challenging not only because one has to conceptualize and operationalize variables that belong to different systems that will in turn be studied in varied contexts, but, more so, because there is a scarce literature that supports this type of research. For instance, studies of societal effects on individual development are almost non-existing in non-Western literature (Petersen, Silbereisen, \& Sörensen, 1996). Consequently, most of the constructs used in our research have been derived from Euroamerican samples that may not make them valid to be transported to another culture without considering the meaning of such constructs in a different context. Dealing with constructs that have not been adapted to another culture may imply the creation of new instruments or the modification of existing ones in order to make the concepts somehow equivalent among the compared cultures.

It is essential that further studies assess development as a culturally adaptive phenomenon (Berry, 1981) that necessarily must include an assessment of the behavior in context (Sameroff, 1986). As a way of dealing with this theoretical and methodological issue, I suggest the inclusion of psychological indices of adaptiveness (emotional and behavioral) when investigating developmental concepts that are prescriptive in nature (or that call for normative judgement) in order to make more meaningful and appropriate conclusions. This may call for the need to generate indigenous psychological knowledge (Kim, 1990). That is, to generate knowledge that include locally relevant concepts and phenomenon. Shina (1983) have argued that many 
concepts that are important in non-Western cultures may be missed if tests and procedures are not developed within a country by its own psychologists.

Although there are recognized constraints on the growth of indigenous knowledge particularly in Third World countries (see e.g., Nsamenang, 1992), these constraints certainly call for collaborative efforts between researchers from advanced industrialized countries and psychologists belonging to the particular culture(s) of interest. This collaboration may result in cross fertilization of ideas and implementation of new approaches, conceptualizations, and even paradigms that would further advance the knowledge and application of human behavior and development across cultures.

\section{Conclusion}

The study described in this dissertation forms part of a larger psychosocial developmental framework that among other goals seeks to enlarge and refine our understanding of the nature of a sense of identity and the process by which it develops including varied sociocultural processes. As such, this dissertation forms part of the preliminary work needed to study how sociocultural processes influence identity formation. This thesis extended the work that has been done on identity by investigating how ethnic/cultural variation influences the formation of an identity as well as by including the concepts of identity satisfaction and dissatisfaction for three identity domains (personal, interpersonal, and world view) at the cross-cultural level. As part of this preliminary work, a framework for conceptualizing identity formation was outlined. In the process of 
operationalizing this framework, measures were developed and experimental procedures were refined for empirically investigating the nature of identity.

This dissertation also sought to enlarge and refine our understanding of the relationship between social institutions and a sense of identity at the theoretical level by proposing a co-constructivist perspective that emphasizes the explanation and understanding of the changing individual in a changing world. In the process of operationalizing this framework, a measure of social institutions was developed and experimental procedures for empirically investigating this relationship were refined.

By having a better understanding of the nature of a sense of identity and the way that social institutions impact identity formation under varied sociocultural contexts, social scientists will be in a better position to assist both theoretically and practically those involved in supporting individuals experiencing this developmental transition and might be able to even suggest social programs and policies with the aim of improving the quality of people's life both at the individual and at the collective level in context. 


\section{References}

Abraham, K.G. (1983). The relation between identity status and locus of control among rural high school students. Journal of Early Adolescence, 3, 257264.

Abraham, K.G. (1986). Ego-identity differences among Anglo-American and Mexican-American adolescents. Journal of Adolescence, $9,151-166$.

Adams, G. R., Abraham, K. G., \& Markstrom, C. (1987). The relations among identity development, self-consciousness, and self-focusing during middle and late adolescence. Developmental Psychology, 23, 292-297.

Adams, G. R., Bennion, L. D., \& Hugh, K. (1987). A revision of the extended version of the objective measure of ego identity status: An identity instrument for use with late adolescents. Journal of Adolescent Research. 1, 183-198.

Adams, G. R., Dyk, P., \& Bennion, L. D. (1990). Parent-adolescent relationships and identity formation. In B. K. Barber \& B. C. Rollins (Eds.), Parent-adolescent relationships. Lanham, MD: University Press of America.

Adams, G.R. \& Fitch, S.A. (1982). Ego stage and identity status development: A cross-sequential analysis. Journal of Personality and Social Psychology, 43, 574-583.

Adams, G.R., Fitch, J., \& Shay, S.A. (1979). Toward the development of an objective measure of ego-identity status. Journal of Youth and Adolescence, $\underline{8}, 223-238$. 
Adams, G. R., Ryan, J. H., Hoffman, J. J., Dobson, W. R., \& Nielsen, E. C. (1985). Ego-identity status, conformity behavior and personality in late adolescence. Journal of Personality and Social Psychology 47, 1091-1104.

Adams, G.R. \& Shea, J.A. (1979). The relationship between identity status, locus of control, and ego development. Journal of Youth and Adolescence, $8,81-89$.

Akhtar, S. (1984). The syndrome of identity diffusion. American Journal of Psychiatry, 141, 1381-1385.

Anonymous (1996). Society, 33, 3.

Aries, E., \& Moorehead, K. (1989). The importance of ethnicity in the development of identity of Black adolescents. Psychological Reports, 65, 75-82.

Bacho, E. (1994). Eriksonian identity exploration and Asian American youth: Comparative investigation of the effects of ethnicity, familial cohesion, and adaptability on identity exploration for Filipino vs European/non-Hispanic Americans. Paper presented at the Society for Research in Adolescence. February 10. San Diego: CA.

Baker, F. (1971). Measures of ego identity: A multitrait multimethod validation. Educational and Psychology Measurement, 31, 165-173.

Bennion, L. D., \& Adams, G. R. (1986). A revision of the extended version of the Objective Measure of Ego Identity Status: An identity instrument for use with late adolescents. Journal of Adolescence Research, 1, 183-197. 
Benson, M. J., Harris, P. B., \& Rogers, C. S. (1992). Identity consequences of attachment to mothers and fathers among late adolescents. Journal of Research on Adolescence, 2, 187-204.

Bernal, G. (1982). Cuban families. In M. McGoldrick, J. K. Pearce, \& J. Giordano (Eds.), Ethnicity and Family Therapy. New York: Guildford.

Berry, J. W. (1981). Developmental issues in the comparative study of psychological differentiation. In R. H. Munroe and B. B. Whiting (Eds.), Handbook of cross-cultural human development. New York: Garland.

Berzonsky, M. D. (1992). A process perspective on identity and stress management. In G. R. Adams, T. P. Gullotta, \& R. Montemayor (Eds.), Adolescent identity formation. Newbury Park, CA: Sage.

Blasi, J. (1995). Moral understanding and moral personality: The process of moral integration. In W. Kurtines and J. Gewirtz (Eds.), Moral development: An introduction. Boston: Allyn \& Bacon.

Botcheva, L. \& Zlatkov, Y. (1994). Youth identity, values and behavior during the transition from totalitarian to democratic society (the case of Bulgaria). Paper presented at the Society for Research in Adolescence, February, San Diego: CA.

Briones, E. (1995). Social institutions and identity formation: The interface between society and the individual. Unpublished master thesis, Florida International University. 
Briones, E. \& Kurtines, W. (1994). Social Institutions Rating. Unpublished measure, Florida International University.

Brislin, R. W. (1986). The wording and translation of research instruments. In W. J. Lonner and J. W. Berry (Eds.), Field methods in crosscultural research. Newbury Park, CA: Sage.

Bronfenbrenner, U. (1986). Ecology of the family as a context for human development: Research perspectives. Developmental Psychology, 22, 723-742.

Bronson, G.W. (1959). Identity diffusion in late adolescence. Journal of Abnormal and Social Psychology, 59, 414-417.

Bunt, M.E. (1962). A study of certain aspects of ego identity as demonstrated by the discrepancy between how an adolescent views himself and how he perceives that others view him. Dissertation Abstracts International, 28, 4474A.

Byrd, B.C. (1971). Identity confusion and related variables as factors in college dropout. Unpublished doctoral dissertation, Rutgers University, New Brunswick, NJ.

Cavarozzi, M. (1992). Beyond transitions to democracy in Latin America. Journal of Latin American studies, 24, 665-685.

Chandler, M. J. (1995). Is this the end of "The Age of Development," or what? or: Please wait a minute, Mr. Post-man. The Genetic Epistemologist, 23, $1-11$. 
Chapman, J.W., \& Nicholls, J.G. (1976). Occupational identity status, occupational preference, and field dependence in Maori and Pakeha boys. Journal of Cross-Cultural Psychology, 7, 61-72.

Christopherson, B. B., Jones, R. M., \& Sales, A. P. (1988). Diversity in reported motivations for substance use as a function of ego-identity development. Journal of Adolescent Research. 3, 141-152.

Clancy, S. M., \& Dollinger, S. J. (1993). Identity, self, and personality: Identity status and the five-factor model of personality. Journal of Research on Adolescence, $3,227-245$.

Cooper, C., Grotevant, H., \& Condon, S. (1983). Individuality and connectedness in the family as a context for adolescent identity formation and role-taking skills. In H. Grotevant \& C. Cooper (Eds.), Adolescent development in the family. San Francisco: Jossey-Bass.

Coté, J. E. \& Hallahar, A. L. (1996)._Generation on hold: Coming of age in the late twentieth century. New York: New York University Press.

Coté, J. E. \& Levine, C. (1983). Marcia and Erikson: The relationship among ego identity status, neuroticism, dogmatism, and purpose in life. Journal of Youth and Adolescence, 12, 43-53.

Coté, J. E. \& Levine, C. (1988a). A critical examination of the ego identity status paradigm. Developmental Review, 8, 147-184.

Coté, J. E. \& Levine, C. (1988b). On critiquing the identity paradigm: A rejoinder to Waterman. Developmental Review. 8, 209-218. 
Coté, J.E. \& Levine, C. (1989). An empirical test of Erikson's theory of ego identity formation. Youth and Society, 20, 388-415.

Coté, J.E. \& Levine, C. (1992). The genesis of the humanistic academic: A second test of Erikson's theory of ego identity formation. Youth and Society. $\underline{23}, 387-410$.

Cotterell, J. (1996). Social networks and social influences in adolescence. London: Routledge.

Defleur, M. L., D'Antonio, W. V., \& DeFleur, L. B. (1981). Sociology: Human society ( $3^{\text {rd }}$ ed.). Dallas: Scott-Foresman.

Dellas, M., \& Jernigan, L. P. (1987). Occupational identity status development, gender comparisons and internal-external locus in first-year Air Force cadets. Journal of Youth and Adolescence, 15, 191-204.

Dellas, M., \& Jernigan, L. P. (1990). Affective personality characteristics associated with undergraduate ego identity formation. Journal of Adolescent Research, 5, 306-324.

Dishion, T., Patterson, G., Stoolmiller, M., \& Skinner, M. (1991). Family, school, and behavioral antecedents to early adolescent involvement with antisocial peers. Developmental Psychology. 27, 172-180.

Donavan, J.M. (1975). Identity status and interpersonal style. Journal of Youth and Adolescence, 8, 223-237.

Erikson, E. H. (1963). Childhood and society (2nd ed.). New York: Norton. Erikson, E. H. (1964). Insight and responsibility. New York: Norton. 
Erikson, E. H. (1968). Identity: Youth and crisis. New York: Norton.

Fitch, S., \& Adams, G. (1983). Ego identity and intimacy status:

Replication and extension. Developmental Psychology, 19, 839-845.

Form, W. (1990). Institutional analysis: An organizational approach. In M.T. Hallinan, D.M. Klein, \& J. Glass (Eds.), Change in societal institutions. New York: Plenum.

Gindling, T. H. (1993). Women's wages and economic crisis in Costa Rica. Economic Development and Cultural Change, 41, 277-297.

Goodman, D. S. G. (1994). Introduction: The political economy of change.

In D. S. G. Goodman \& B. Hooper (Eds.), China's quiet revolution: New interactions between state and society. Melbourne, Australia: Longman Cheshire.

Grotevant, H. D. \& Adams, G. R. (1984). Development of an objective measure to assess ego identity in adolescence: Validation and replication. Journal of Youth and Adolescence, 13, 419-438.

Grotevant, H. D., \& Cooper, C. (1985). Patterns of interaction in family relationships and the development of identity exploration in adolescence. Child Development, 56, 415-428.

Haberman, P. W. (1976). Psychiatric symptoms among Puerto Ricans in Puerto Rico and New York city. Ethnicity, 5, 133-144.

Haberman, S. J. (1978). Analysis of qualitative data (Vol. 1). London: Academic Press. 
Hair, J. S., Anderson, R. E., \& Tatham, R. L. (1987)._Multivariate data analysis. New York: Macmillan.

Hallinan, M.T., Klein, D.M., \& Glass, J. (1990) (Eds.). Change in Societal Institutions. New York: Plenum.

Hamilton, S. H., \& Darling, N. (1996). Mentors in adolescents' lives. The social world of adolescents: A sociological perspective. In K. Hurrelmann \& S. F. Hamilton (Eds.), Social problems and social contexts in adolescence (pp. 199215). Hawthorne, NY: Aldine De Gruyter.

Hamner, T. J., \& Turner, P. H. (1990). Parenting in contemporary society. ( $2^{\text {nd }}$ ed.). Needham Heights, MS: Allyn and Bacon.

Harkness, S., \& Super, C. M. (1996) (Eds.). Parents cultural beliefs systems: Their origins, expressions, and consequences. New York: Guildford.

Harwood, R. L., Miller, J. G., \& Irizarry, N. L. (1995). Culture and attachment: Perceptions of the child in context. New York: Guildford.

Hauser, S. T. (1972). Adolescent self-image development. Archives of General Psychiatry, 27, 537-541.

Hauser, S. T., Powers, S. I., Noam, G. G., Jacobson, A. M., Weiss, B., \& Follansbee, D. J. (1984). Familial contexts of adolescent ego development._ Child Development, 55, 195-213.

Herrick, J. E. (1977). Theory building for basic institutional change. San Francisco: R and E Research Associates. 
Hunt, E.F., \& Colander, D.C. (1987). Social science: An introduction to the study of society, 6th Ed. New York: MacMillan.

Hurrelmann, K. (1996). The social world of adolescents: A sociological perspective. In K. Hurrelmann \& S. F. Hamilton (Eds.), Social problems and social contexts in adolescence (pp. 39-62). Hawthorne, NY: Aldine De Gruyter. Interamerican Development Bank (1989). Economic and social progress in Latin America: 1989 report. Washington, D.C.: Inter-American Development Bank.

Josselson, R. (1989). Identity formation in adolescence: Implications for young adulthood. In S. C. Feinstein (Ed.), Adolescent Psychiatry (Vol. 16). Chicago: University of Chicago Press.

Kim, U. (1990). Indigenous psychology: Science and applications. In R. W. Brislin (Ed.), Applied cross-cultural psychology. Newbury Park, CA: Sage.

Klecka, W. (1980). Discriminant analysis. Beverly Hills: Sage.

Knight, J. (1992). Institutions and social conflict. New York: Cambridge University Press.

Kroger, J. (1993). The role of historical context in the identity formation process of late adolescence. Paper presented at the Biennial Meeting of the Society for Research in Child Development, New Orleans.

Kroger, J. (1996). Identity regression and development. Journal of Adolescence. 19, 203-222. 
Kroger, J., \& Haslett, S. (1988). Separation-individuation and ego identity status in late adolescence: A two-year longitudinal study. Journal of Youth and Adolescence, $17,59-79$.

Kurtines, W. (1984). Moral behavior as rule governed behavior: A psychosocial role theoretical approach to moral behavior and development. In W. Kurtines \& J. L. Gewirtz (Eds.), Morality, moral behavior, and moral development. New York: John Wiley \& Sons, 303-324.

Kurtines, W. (1987). Psychosocial theory as a nomotic science. In W. Kurtines \& J. L. Gewirtz (Eds.), Moral development through social interaction. New York: John Wiley \& Sons.

Kurtines, W. M. (1996). Human behavior and development: A coconstructivist perspective. Unpublished manuscript, Florida International University.

Kurtines, W. M., Berman, S. L., Itell, A., \& Williamson, S. (1995). Moral development: A co-constructivist perspective. In W. M. Kurtines \& J. L. Gewirtz (Eds.), Moral development: An introduction. Boston, MA: Simon \& Schuster.

Kvale, S. (1992). Psychology and postmodernism. London: Sage.

Lancy, D. F. (1996). Playing on the mother-ground: Cultural routines for children's development. New York: Guildford.

Lehoucq, F. E. (1996). The institutional foundations of democratic cooperation in Costa Rica. Journal of Latin American Studies, 28, 329-355. 
Lyotard, J-F. (1979). Excerpts from the postmodern condition: A report on knowledge. Minneapolis: University of Minnesota Press.

Macionistl, J. J. (1997). Perspectives on culture. In M. A. Escotet (Ed.), Cultural and social foundations of education: An interdisciplinary approach. Needham Heights, MA: Simon \& Schuster.

Madeley, J. (1995). The return of Swedish social democracy: Phoenix or ostrich? West European Politics, 18, 422-429.

Marcia, J. E. (1966). Development and validation of ego identity status. Journal of Personality and Social Psychology. 3, 551-558.

Marcia, J.E. (1980). Identity in adolescence. In J. Adleson (Ed.), Handbook of Adolescent Psychology. New York: Wiley.

Marcia, J. E. (1992). Ego psychoanalytic perspectives on identity. Paper presented at the meeting of the American Psychological Association, Washington, D.C., August.

Marcia, J.E., Waterman, A.S., Matteson,D.R., Archer, S.L., Orlofsky, J.L. (1993). Ego Identity: A Handbook for Psychosocial Research. New York: Springer-Verlag.

Markstrom-Adams, C., Ascione, F. R., Braegger, D., \& Adams, G. (1987). A comparison of psychosocial maturity between four ethnic groups during middle adolescence. Paper presented at the biennial meetings of the Society of Research on Child Development, Baltimore, MD, April. 
McClain, E.W. (1975). An Eriksonian cross-cultural study of adolescent development. Adolescence. 10, 527-541.

McDavid, J. H. \& Harari, J. W. (1974). Psychology and social behavior. New York: Harper-Row.

Meeus, W. (1996). Toward a psychosocial analysis of adolescent identity: An evaluation of the epigenetic theory (Erikson) and the identity status model (Marcia). In K. Hurrelmann \& S. F. Hamilton (Eds.), Social problems and social contexts in adolescence: Perspectives across boundaries (pp. 83-104). New York: Aldine De Gruyter.

Mesa-Lago, C. (1997). Social welfare reform in the context of economicpolitical liberalization: Latin American cases. World Development, 25 (4), 497$518)$.

Matteson, D.R. (1977). Exploration and commitment: Sex differences and methodological problems in the use of identity status categories. Journal of Youth and Adolescence, 6, 353-374.

Mayer, R.R. (1982). Social Science and Institutional Change. New Brunswick, NJ: Transaction Books.

Murray, J.B. (1964). The identity image of college students. Psychological Reports, 14, 267-271.

Muss, R.E. (1975). Theories of adolescence. New York: Random House. Niemi, T. (1988). Problems among students seeking mental health care. Journal of American College Health, 36, 353-354. 
Nsamenang, A. B. (1992). Human development in cultural context: A third world perspective. Newbury Park, CA: Sage.

Nugent, J. K., Lester, B. M., \& Brazelton, T. B. (Eds.) (1989). The cultural context of infancy. Norwood, New Jersey: Ablex.

Ochse, R. \& Plug, C. (1986). Cross-cultural investigation of the validity of Erikson's theory of personality development. Journal of Personality and Social Psychology, 50, 1240-1252.

Pereira, L. C. B. (1996). Economic crisis and state reform in Brazil: Toward a new interpretation of Latin America. Boulder, CO: Rienner.

Pereira, A. Jr., \& Heringer, R. (1994). Brazil. In Hurrelmann, K. (Ed.), International handbook of adolescence (pp. 65-76). Westport, CT: Greenwood.

Petersen, A. C., Silbereisen, R. K., Sörensen, S. (1996). Adolescent development: A global perspective. The social world of adolescents: A sociological perspective. In K. Hurrelmann \& S. F. Hamilton (Eds.), Social problems and social contexts in adolescence (pp. 39-62). Hawthorne, NY: Aldine De Gruyter.

Phinney, J. S. (1992). The multigroup ethnic identity measure: A new scale for use with diverse groups. Journal of Adolescent Research, 7, 156-176.

Phinney, J. S. (1993). A three-stage model of ethnic identity development in adolescence. In M. E. Bernal and G. P. Knight (Eds.), Ethnic identity: formation and transmission among Hispanics and other minorities. New York: State University of New York Press. 
Phinney, J. S. (1989). Stages of ethnic identity development in minority group adolescents. Journal of Early Adolescence, 9, 34-49.

Phinney, J. S., \& Alipuria, L. L. (1990). Ethnic identity in college students from four ethnic groups. Journal of Adolescence, 13, 171-183.

Phinney, J. S., \& Chavira, V. (1992). Ethnic identity and self-esteem: An exploratory longitudinal study. Journal of Adolescence. 15, 271-281.

Poole, M. E., Cooney, G. H. \& Cheong, A. C. S. (1986). Adolescent perceptions of family cohesiveness, autonomy, and independence in Australia and Singapore. Journal of Comparative Family Studies, 17, 311-332.

Prager, K. J. (1986). Identity development, age, and college experience in women. Journal of Genetic Psychology, 147, 31-36.

Rasmussen, J.E. (1964). The relationship of ego identity to psychosocial effectiveness. Psychological Reports, 15, 815-825.

Roe, K., Bjurström, E., \& Fornäs, J. (1994). Sweden. In Hurrelman, K. (Ed.), International handbook of adolescence (pp. 374-385). Westport, CT: Greenwood.

Rorty, R. (1992). Consequences of pragmatism: Essays 1972-1980. Minneapolis: Univesity of Minnesota Press.

Rotheram-Borus, M. J. (1989). Ethnic differences in adolescents' identity status and associated behavior problems. Journal of Adolescence. 12, 361-374.

Samerof, A. J. (1983). Developmental systems: Contexts and evolution. Handbook of child psychology: Vol. 1. New York: Wiley. 
Schmaltz, J., Kurtines, W., Berman, S., \& Briones, E. (1994). Identity Domains Scale. Unpublished measure, Florida International University.

Sennett, R. (1997). The new capitalism. Social Research, 64(2), 161181.

Shina, D. (1983). Human assessment in Indian context. In S. H. Irvine \& J. W. Berry (Eds.), Human assessment and cultural factors. New York: Plenum.

Shwalb, D. W., \& Shwalb, B. (1996) (Eds.). Japanese childrearing: Two generations of scholarship. New York: Guildford.

Simmons, D.D. (1970). Development of an objective measure of identity achievement status. Journal of Projective Techniques and Personality Assessment, 34, 241-244.

Slugoski, B. R., Marcia, J. E., \& Koopman, R. F. (1984). Cognitive and social interactional characteristics of ego identity statuses in college males._ Journal of Personality and Social Psychology, 47, 646-661.

Smith, M., Canter, W., \& Robin, A. (1989). A path analysis of an adolescent drinking behaviour model derived from problem behavior theory. Journal of Studies on Alcohol, 50, 128-142.

Stigler, J. W., Shweder, R. A., \& Herdt, G. (1990) (Eds.)._Cultural psychology: Essays on comparative human development. Cambridge, NY: Cambridge University Press.

Streitmattter, J. L. (1988). Ethnicity as a mediating variable of early adolescent identity development. Journal of Adolescence, 11, 335-346. 
Svallfors, S. (1995). The end of class politics? Structural cleavages and attitudes to Swedish welfare policies. Acta Sociologica, 38, 53-75.

Taylor, R. D., \& Oskay, G. (1995). Identity formation in Turkish and American late adolescents. Journal of Cross-Cultural Psychology. 26, 8-22.

Tesch, S. A. \& Cameron, K. A. (1987). Openness to experience and development of adult identity. Journal of Personality, 55, 615-630.

Telles, P. E. (1983). A comparison of patterns of ego identity formation in Mexican American and Anglo American late adolescents. Unpublished dissertation, Boston University School of Education.

Unger, J. \& McCormick, B. L. (1996). Introduction. In B. L. McCormick \& J. Unger (Eds.), China after socialism: In the footsteps of Eastern Europe or East Asia? Armonk, NY: M. E. Sharpe.

U.S. Bureau of the Census (1993). Current Population Reports, P23-183. Hispanic American today. Washington: U.S. Government Printing Office.

Van de Vijver, F. J. R., \& Poortinga, Y. H. (1991). Testing across culture. In R. K. Hambleton and J. N. Zaal (Eds.), Advances in educational and psychological testing. Boston: Kluwer Academic Publishers.

Wallace-Broscious, A., Serafica, F. C., \& Osipow, S. H. (1994). Adolescent career development: Relationships to self-concept and identity status. Journal of Research on Adolescence, 4, 127-149. 
Waterman, A. S. (1982). Identity development from adolescence to adulthood: An extension of theory and a review of research. Developmental Psychology, 18, 341-358.

Waterman, A.S. (1984). Identity formation: Discovery or creation? Journal of Early Adolescence, 4, 329-341.

Waterman, A.S. (1985). Identity in the context of adolescent psychology. In A.S. Waterman (Ed.), Identity in adolescence: Processes and contents. San Francisco: Jossey-Bass.

Waterman, A. S. (1988). Identity status theory and Erikson's theory: communalities and differences. Developmental Review, 8, 185-208.

Waterman, A.S. (1992). Identity as an aspect of optimal psychological functioning. In G.R. Adams, T.P. Gullotta \& R. Montemayor (Eds.) Adolescent Identity Formation. Newbury Park, CA: Sage, 50-72.

Waterman, A., Geary, P., \& Waterman, M. (1974). A longitudinal study of changes in ego identity status from the freshman to the senior year at college. Developmental Psychology, 10, 387-392.

Waterman, A., \& Goldman, J. (1976). A longitudinal study of ego identity development at a liberal arts college. Journal of Youth and Adolescence, 5, 361369.

Waterman, A., \& Waterman, M. (1971). A longitudinal study of changes in ego identity status during the freshman year at college. Developmental Psychology, 5, 167-173. 
Watson, M. T., \& Protinsky, H. (1991). Identity status of Black

adolescents: An empirical investigation. Adolescence, 26, 963-966.

Yunling, Z. (1995). China in the post-cold war era. In Holm, H-H. \& G.

Sorensen (Eds.), Whose world order? Uneven globalization and the end of the cold war. Boulder, CO: Westview.

Zarate, J. C. (1994). Forging democracy: A contemporary study of the effects of U.S. foreign policy on Central American democratization. Maryland: University Press of America. 
APPENDIX A 


\title{
Identity Domain Scale ${ }^{2}$
}

\author{
Jamie A. Schmaltz, William M. Kurtines,
}

Steven L. Berman, Ervin Briones

Florida Internaional University

\section{Draft}

${ }^{2}$ Rev.: 1/94; Printed: 12/7/97

For information about this survey contact the authors at:

FIU Identity Project

Department of Psychology

Florida International University

Miami, Florida 33199

Telephone: (305) 348-3941

Fax: (305) 348-3879

E-Mail:

WANDERMA@SERVAX.FIU.EDU

KURTINES@SERVAX.FIU.EDU 


\section{Identity Domain Scale}

Directions: We are interested in your thoughts and feelings about a variety of issues relating to your identity. Identity has to do with your sense of self. More specifically, we will be asking you about your satisfaction or dissatisfaction with the direction of your life. This survey has nine parts. Each part asks about a different content area related to your sense of self. You will be asked to describe what is good or positive and what is distressful or troublesome in each content area and how much you have thought about each of these areas. Please write your answers on both the bubble sheet and the description sheet provided. DO NOT WRITE ON THIS QUESTIONAIRE. Please answer every question. 


\section{Biographical Data}

Please record your answers on the information section of your bubble answer sheet.

Sex: Male Female

Grade: $\begin{array}{llllll}7 & 8 & 9 & 10 & 11 & 12\end{array}$

College: $\quad \begin{array}{ll}13 & =\text { Freshman } \\ 14 & =\text { Sophmore } \\ 15 & =\text { Junior } \\ 16 & =\text { Senior }\end{array}$

Age: (Indicate your age under "YR." on the answer sheet)

Race: (Indicate your race under " $\mathrm{K}$ " of special codes on the answer sheet)

$$
\begin{aligned}
& 1=\text { White } \\
& 2=\text { Black (Non-Hispanic) } \\
& 3=\text { Hispanic } \\
& 4=\text { Asian } \\
& 5=\text { Native American } \\
& 6=\text { Other }
\end{aligned}
$$

Marital Status: (Indicate your marital status under " $\mathrm{L}$ " of special codes on the answer sheet)

$$
\begin{aligned}
& 1=\text { Single } \\
& 2=\text { Married } \\
& 3=\text { Divorced } \\
& 4=\text { Widowed }
\end{aligned}
$$




\section{Domain I:}

\section{Personal Development}




\section{Part $\boldsymbol{A}$ asks about career goals.}

\section{PART A: SECTION I}

We would like you to identify and describe what, if anything, you consider good or positive about your thoughts, feelings, practices, experiences, or any other issues that are important to you about your career goals. Below is a list of content features with examples. Look them over carefully and identify the most important area of positive experiences for you. Enter the code letter on the bubble sheet and describe this area of importance in your own words on the description sheet provided.

\section{Positive Content Features \\ (with examples)}

Code (A) DECISION: The actual decision itself and/or the process of making a decision.

EX: $\quad-I$ know what I want to pursue -I want to be a teacher

Code (B) ACHIEVEMENT: The achievement of your aspirations or goals.

-I believe I can accomplish my goals

-I am currently in a job that will help me in the future

Code (C) REWARDS: The rewards related to your decision -I will be challenged and satisfied -I will be able to help children

Code (D) NO ISSUES: This is not an important issue for you

1. Go to your description sheet and record your code letter and your description of the important issues.

2. How positive is this issue to you at this moment?

$\begin{array}{lllll}\text { Not } & \text { A little } & & \text { Very } & \text { Extremely } \\ \text { Positive } & \text { Positive } & \text { Positive } & \text { Positive } & \text { Positive }\end{array}$




\section{PART A: SECTION II \\ Distressful Content Features \\ (with examples)}

We would like you to identify and describe what, if anything, you consider distressful and troublesome about your thoughts, feelings, practices, experiences, or any other issues that are important to you about your career goals. Below is a list of content features with examples. Look them over carefully and identify the most important area of distressful experiences for you. Enter the code letter on the bubble sheet and describe this area of importance in your own words on the description sheet provided.

Code (A) DECISION: The actual decision itself and/or the process of making a decision.

-I don't know what I want to pursue

-I am confused about my future

Code (B) ACHIEVEMENT: The achievement of your aspirations or goals.

-I may not have the grades to get into graduate school to become a teacher

-I may not be able to get the experience that will help me in the future

Code (C) REWARDS: The rewards related to your decision

-I may not be challenged and satisfied

-I may not be able to have the financial advancement I want

Code (D) NO ISSUES:This is not an important issue for you

3. Go to your description sheet and record your code letter and your description of the important issues.

4. How distressful is this issue to you at this moment?

$\begin{array}{clccc}\text { Not } & \text { A little } & & \text { Very } & \text { Extremely } \\ \text { Distressful } & \text { Distressful } & \text { Distressful } & \text { Distressful } & \text { Distressful } \\ \text { A } & \text { B } & \text { C } & \text { D } & \text { E }\end{array}$

5. Have you had distress about this issue for longer than three months?
(A) Yes
(B) No

6. Have you had distress about this issue which significantly interfered with your daily living?
(A) Yes
(B) No

7. Of the following statements, which best describes your current thoughts and feelings with respect to your career goals:

(A). I am not sure about my career goals and I haven't spent much time questioning or thinking about it.

(B). After a lot of self-examination and experience, I have established a clear sense of my career goals.

(C). I have never really questioned my career goals because I have always known what I wanted to do.

(D). After a lot of self-examination and experience, I have NOT established a clear sense of my career goals. 


\title{
Part $B$ asks about your sense of self
}

\author{
PART B: SECTION I \\ Positive Content Features \\ (with examples)
}

We would like you to identify and describe what, if anything, you consider good or positive about your thoughts, feelings, practices, experiences, or any other issues that are important to you about your sense of self. Below is a list of content features with examples. Look them over carefully and identify the most important area of positive experiences for you. Enter the code letter on the bubble sheet and describe this area of importance in your own words on the description sheet provided.

Code (A) SELF ESTEEM: The feelings you have about yourself and who you are.

$$
\begin{aligned}
& \text {-I feel good about myself } \\
& \text {-I am a confident person }
\end{aligned}
$$

Code (B) BODY IMAGE: The thoughts and feelings you have about the way you perceive your physical appearance.

-I think I am very attractive and I like the way I look

-I am physically fit which makes me feel good about how I look

Code (C) SELF IMAGE: The thoughts and feelings that reflect your personal qualities.

-I am a positive person and I know what I want in life

-I am a caring and loving person

Code (D) NO ISSUES: This is not an important issue for you

8. Go to your description sheet and record your code letter and your description of the important issues.

9. How positive is this issue to you at this moment?

$\begin{array}{lllll}\text { Not } & \text { A little } & & \text { Very } & \text { Extremely } \\ \text { Positive } & \text { Positive } & \text { Positive } & \text { Positive } & \text { Positive }\end{array}$
A
B
$\mathrm{C}$
D
E 


\section{PART B: SECTION II \\ Distressful Content Features \\ (with examples)}

We would like you to identify and describe what, if anything, you consider distressful or troublesome about your thoughts, feelings, practices, experiences, or any other issues that are important to you about your sense of self. Below is a list of content features with examples. Look them over carefully and identify the most important area of distressful experiences for you. Enter the code letter on the bubble sheet and describe this area of importance in your own words on the description sheet provided.

Code (A) SELF ESTEEM: The feelings you have about yourself and who you are.

-I sometimes feel inadequate

-I feel that I may not be strong enough to acheive my goals

Code (B) BODY IMAGE: The thoughts and feelings you have about the way you perceive your physical appearance.

-I don't like my body

-I am overweight for the way I would like to look

Code (C) SELF IMAGE: The thoughts and feelings that reflect your personal qualities. -I am easlily intiminated and pushed to do things I don't agree with -I am pesimistic and I usually see the bad side of situations

Code (D) NO ISSUES: This is not an important issue for you

10. Go to your description sheet and record your code letter and your description of the important issues.

11. How distressful is this issue to you at this moment?

$\begin{array}{clccc}\text { Not } & \text { A little } & & \text { Very } & \text { Extremely } \\ \text { Distressful } & \text { Distressful } & \text { Distressful } & \text { Distressful } & \text { Distressful } \\ \text { A } & \text { B } & \text { C } & \text { D } & \text { E }\end{array}$

12. Have you had distress about this issue for longer than three months?
(A) Yes
(B) No

13. Have you had distress about this issue which significantly interfered with your daily living?
(A) Yes
(B) No

14. Of the following statements, which best describes your current thoughts and feelings with respect to your sense of self:

(A). After a lot of self-examination and experience, I have NOT established a clear sense of self.

(B). After a lot of self-examination and experience, I have established a clear sense of self.

(C). I have never really questioned my sense of self because I have always known who I am.

(D). I am not sure about how I feel about my sense of self and I haven't spent much time questioning or thinking about it. 


\title{
Part $C$ asks about long-term life goals.
}

\author{
PART C: SECTION I \\ Positive Content Features \\ (with examples)
}

We would like you to identify and describe what, if anything, you consider good or positive about your thoughts, feelings, practices, experiences, or any other issues that are important to you about your long-term life goals. Below is a list of content features with examples. Look them over carefully and identify the most important area of positive experiences for you. Enter the code letter on the bubble sheet and describe this area of importance in your own words on the description sheet provided.

Code (A) DECISION: The actual decision itself and/or the process of making it.

-I feel I have made a positive decision

-Someday I would like to be a good wife and mother

Code (B) ACHIEVEMENT: The achievement of your aspirations or goals.

-I think I have the personal qualities to achieve my goals

-I am trying to be a well-rounded individual

Code (C) REWARDS: The rewards related to your decision

-I want to make a difference in the world

-I want to have independence and security

Code (D) NO ISSUES: This is not an issue for you

15. Go to your description sheet and record your code letter and your description of the important issues.

16. How positive is this issue to you at this moment?

$\begin{array}{lllll}\text { Not } & \text { A little } & & \text { Very } & \text { Extremely } \\ \text { Positive } & \text { Positive } & \text { Positive } & \text { Positive } & \text { Positive }\end{array}$
A
B
C
D
E 


\section{PART C: SECTION II}

\section{Distressful Content Features \\ (with examples)}

We would like you to identify and describe what, if anything, you consider distressful or troublesome about your thoughts, feelings, practices, experiences, or any other issues that are important to you about your long-term life goals. Below is a list of content features with examples. Look them over carefully and identify the most important area of distressful experiences for you. Enter the code letter on the bubble sheet and describe this area of importance in your own words on the description sheet provided.

Code (A) DECISION: The actual decision itself and/or the process of making it

-I have not decided what to do with my life

-I am not sure if my choice is the right thing for me

Code (B) ACHIEVEMENT: The achievement of your aspirations or goals.

-I feel I won't be able to achieve my goals

-I worry that I may never get married

Code (C) REWARDS: The rewards related to your decision

-I may not be challenged and satisfied

-My children may become rebellious

Code (D) NO ISSUES: This is not an issue for you

17. Go to your description sheet and record your code letter and your description of the important issues.

18. How distressful is this issue to you at this moment?

$\begin{array}{clccc}\text { Not } & \text { A little } & & \text { Very } & \text { Extremely } \\ \text { Distressful } & \text { Distressful } & \text { Distressful } & \text { Distressful } & \text { Distressful } \\ \text { A } & \text { B } & \text { C } & \text { D } & \text { E }\end{array}$

19. Have you had distress about this issue for longer than three months?
(A) Yes
(B) No

20. Have you had distress about this issue which significantly interfered with your daily living?
(A) Yes
(B) No

21. Of the following statements, which best describes your current thoughts and feelings with respect to your long-term life goals:

(A). I am not sure about my long-term life goals and I haven't spent much time questioning or thinking about it.

(B). After a lot of self-examination and experience, I have NOT established a clear sense of my long-term life goals.

(C). I have never really questioned my long-term life goals because I have always known what I wanted out of life.

(D). After a lot of self-examination and experience, I have established a clear sense of my longterm life goals. 


\section{Domain II:}

\section{Interpersonal Development}




\section{Part $D$ asks about friendships.}

\section{PART D: SECTION I \\ Positive Content Features \\ (with examples)}

We would like you to identify and describe what, if anything, you consider good or positive about your thoughts, feelings, practices, experiences, or any other issues that are important to you about your friendships. Below is a list of content features with examples. Look them over carefully and identify the most important area of positive experiences for you. Enter the code letter on the bubble sheet and describe this area of importance in your own words on the description sheet provided.

Code (A) PERSON QUALITIES: The qualities of the persons involved in the friendship

-My friends understand me

-My friends are loyal

Code (B) FRIENDSHIP QUALITIES: The qualities of the friendship itself

-We both know we can count on each other

-My friends and I have mutual respect and honesty

Code (C) RELATIONSHIP PROCESSES: The process \& interactions related to the friendships.

-My friend gives me good advice when I need it most

-I enjoy sharing my feelings and experiences

Code (D) NO ISSUES: This is not an issue for you

22. Go to your description sheet and record your code letter and your description of the important issues.

23. How positive is this issue to you at this moment?

$\begin{array}{lllll}\text { Not } & \text { A little } & & \text { Very } & \text { Extremely } \\ \text { Positive } & \text { Positive } & \text { Positive } & \text { Positive } & \text { Positive }\end{array}$

A

B

$\mathrm{C}$

D

$\mathrm{E}$ 


\section{PART D: SECTION II \\ Distressful Content Features \\ (with examples)}

We would like you to identify and describe what, if anything, you consider distressful or troublesome about your thoughts, feelings, practices, experiences, or any other issues that are important to you about your friendships. Below is a list of content features with examples. Look them over carefully and identify the most important area of distressful experiences for you. Enter the code letter on the bubble sheet and describe this area of importance in your own words on the description sheet provided.

Code (A) PERSON QUALITIES: The qualities of the persons involved in the friendship -My friend can be secretive sometimes

-My friends talk behind my back

Code (B) FRIENDSHIP QUALITIES: The qualities of the friendship itself

-My friends and I have not been able to accept each other's faults

-It can be difficult to have friendships with people of the opposite sex

Code (C) RELATIONSHIP PROCESS: The process \& interactions related to the friendships.

-It is not fair that one of us is giving more than the other

-Sometimes lying interferes with the ability for friends to share intimate secrets

Code (D) NO ISSUES: This is not an issue for you

24. Go to your description sheet and record your code letter and your description of the important issues.

25. How distressful is this issue to you at this moment?

\begin{tabular}{|c|c|}
\hline $\begin{array}{l}\text { Not } \\
\text { Distressful }\end{array}$ & $\begin{array}{l}\text { A little } \\
\text { Distressful }\end{array}$ \\
\hline
\end{tabular}

A

B

$\mathrm{C}$

$\mathrm{D}$

$\mathrm{E}$

26. Have you had distress about this issue for longer than three months?
(A) Yes
(B) No

27. Have you had distress about this issue which significantly interfered with your daily living?
(A) Yes
(B) No

28. Of the following statements, which best describes your current thoughts and feelings with respect to your friendships:
(A). I am still exploring what types of friends are right for me at this point in my life.
(B). After a lot of self-examination and experience, I have established a clear sense of what type of friendships I want and I choose my friends accordingly.
(C). I am not really sure about what I want in a friend and I have not given it much thought.
(D). I know what I want in a friend and I have never really had to question it. 


\section{Part $E$ asks about belonging to groups. \\ PART E: SECTION I}

\section{Positive Content Features \\ (with examples)}

We would like you to identify and describe what, if anything, you consider good or positive about your thoughts, feelings, practices, experiences, or any other issues that are important to you about your group involvement. Below is a list of content features with examples. Look them over carefully and identify the most important area of positive experiences for you. Enter the code letter on the bubble sheet and describe this area of importance in your own words on the description sheet provided.

Code (A) GROUP QUALITIES: The qualities of the group.

-The group I belong to is very supportive

-The group I belong to gives me a sense of belonging

Code (B) INDIVIDUAL QUALITIES: The qualities of the persons involved in the group.

-I feel I can contribute to the goals of my group

-The people in my group and myself have similar interests

Code (C) GROUP PROCESS: Processes or interactions of belonging/not belonging to a group. -Provides social interaction which fosters communication

-We work well together as a team and have a lot of cooperation

Code (D) NO ISSUES: This is not an issue for you

29. Go to your description sheet and record your code letter and your description of the important issues.

30. How positive is this issue to you at this moment?

Not

Positive

A
A little

Positive

B
Positive

$\mathrm{C}$
Very

Positive
Extremely

Positive

D

E 


\section{PART E: SECTION II \\ Distressful Content Features \\ (with examples)}

We would like you to identify and describe what, if anything, you consider distressful or troublesome about your thoughts, feelings, practices, experiences, or any other issues that are important to you about your group involvement. Below is a list of content features with examples. Look them over carefully and identify the most important area of distressful experiences for you. Enter the code letter on the bubble sheet and describe this area of importance in your own words on the description sheet provided.

Code (A) GROUP QUALITIES: The qualities of the group.

-Sometimes my group is too militant

-Sometimes I disagree with the group's goals

Code (B) INDIVIDUAL QUALITIES: The qualities of the persons involved in the group.

-Certain people in the group do not represent the group's ideals

-Some people in the group sit back and let others do all the work

Code (C) GROUP PROCESS: Processes or interactions of belonging/not belonging to a group.

-Sometimes I feel isolated and alone even though I am a member of the group

-I feel not everyone has a voice in the group

Code (D) NO ISSUES: This is not an issue for you

31. Go to your description sheet and record your code letter and your description of the important issues.

32. How distressful is this issue to you at this moment?

$\begin{array}{clccc}\text { Not } & \text { A little } & & \text { Very } & \text { Extremely } \\ \text { Distressful } & \text { Distressful } & \text { Distressful } & \text { Distressful } & \text { Distressful } \\ \text { A } & \text { B } & \text { C } & \text { D } & \text { E }\end{array}$

33. Have you had distress about this issue for longer than three months?
(A) Yes
(B) No

34. Have you had distress about this issue which significantly interfered with your daily living?
(A) Yes
(B) No

35. Of the following statements, which best describes your current thoughts and feelings with respect to belonging to groups:

(A). I haven't spent much time questioning or thinking about what type of groups I should belong to or if I should belong to groups at all.

(B). I know what type of group involvement is best for me and I have never really had to question it.

(C). After a lot of self-examination and experience, I still have NOT established a clear sense of the type and level of group involvement would be best for me.

(D). After a lot of self-examination and experience, I have established a clear sense of the type and level of group involvement would be best for me. 


\section{Part $F$ asks about gender and sexuality issues. PART F: SECTION I}

\section{Positive Content Features}

We would like you to identify and describe what, if anything, you consider good or positive about your thoughts, feelings, practices, experiences, or any other issues that are important to you about your gender and sexuality. Below is a list of content features with examples. Look them over carefully and identify the most important area of positive experiences for you. Enter the code letter on the bubble sheet and describe this area of importance in your own words on the description sheet provided.

(with examples)

Code (A) GENDER: The role and function that you play in society due to your gender.

-I feel positive that I am able to carry a child

-I benefit from the status that society assigns me

Code (B) SEXUALITY: The role that sexuality play in your life.

-I understand my sexuality and I am comfortable with myself

-Accepting my sexuality preference has given me a sense of power in my life

Code (C) SEX: The role that sex plays in your life

-I feel positive about my decision to become sexually active

-I have had very positive sexual experiences

Code (D) NO ISSUES: This is not an issue for you

36. Go to your description sheet and record your code letter and your description of the important issues.

37. How positive is this issue to you at this moment?

Not

Positive

A
A little

Positive

B
Positive

C
Very

Positive

D
Extremely

Positive 


\section{PART F: SECTION II \\ Distressful Content Features \\ (with examples)}

We would like you to identify and describe what, if anything, you consider distressful or troublesome about your thoughts, feelings, practices, experiences, or any other issues that are important to you about your sexulaity and gender. Below is a list of content features with examples. Look them over carefully and identify the most important area of distressful experiences for you. Enter the code letter on the bubble sheet and describe this area of importance in your own words on the description sheet provided.

Code (A) GENDER: The role and function that you play in society due to your gender.

-I hate the physical limitations associated with my gender

-Because of my gender, I am often a victim of discrimination and sexual harassment

Code (B) SEXUALITY: The role that sexuality play in your life.

-I am worried about the social implications of being a homosexual

-I am not sure about my sexual preference

Code (C) SEX: The role that sex plays in your life

-I feel I am too occupied by sexual thoughts

-I'm not sure I want to become sexually active

Code (D) NO ISSUES: This is not an issue for you

38. Go to your description sheet and record your code letter and your description of the important issues.

39. How distressful is this issue to you at this moment?

$\begin{array}{clccc}\text { Not } & \text { A little } & & \text { Very } & \text { Extremely } \\ \text { Distressful } & \text { Distressful } & \text { Distressful } & \text { Distressful } & \text { Distressful } \\ \text { A } & \text { B } & \text { C } & \text { D } & \text { E }\end{array}$

40. Have you had distress about this issue for longer than three months?
(A) Yes
(B) No

41. Have you had distress about this issue which significantly interfered with your daily living?
(A) Yes
(B) No

42. Of the following statements, which best describes your current thoughts and feelings with respect to gender and sexuality issues:

(A). I have thought about the many issues I must deal with in relation to my gender and my sexuality, but I have not yet defined the role I will take.

(B). I am not sure about my gender role or sexuality and I have not spent much time questioning or thinking about it.

(C). I have never really questioned my gender role or my sexuality because I have used my parents as role models.

(D). I have thought a lot about different gender roles or my sexuality and I have chosen the one that is best for me. 


\section{Domain III:}

\section{Beliefs About the World}




\section{Part $G$ asks about religious issues. \\ PART G: SECTION I \\ Positive Content Features \\ (with examples)}

We would like you to identify and describe what, if anything, you consider good or positive about your thoughts, feelings, practices, experiences, or any other issues that are important to you about your religion. Below is a list of content features with examples. Look them over carefully and identify the most important area of positive experiences for you. Enter the code letter on the bubble sheet and describe this area of importance in your own words on the description sheet provided.

Code (A) BELIEFS: The influence of your religious beliefs on your life -I believe in God

-Due to my choice, I don't have to depend on religion or God for strength

Code (B) MEANING/DIRECTION: The implications that your beliefs on the quality of your life. -My religion teaches me how to live my daily life

-My religion gives me a perspective for what is important in life

Code (C) RULES AND PRACTICES:Implications that your beliefs have on governing your life. -I enjoy going to my place of worship

-I feel free from restrictions laid down by religions

Code (D) NO ISSUES: This is not an issue for you

43. Go to your description sheet and record your code letter and your description of the important issues.

44. How positive is this issue to you at this moment?

Not

Positive

A little

A

B

Positive

Very

Positive

Extremely

Positive

C

D

E 


\section{PART G: SECTION II \\ Distressful Content Features \\ (with examples)}

We would like you to identify and describe what, if anything, you consider distressful or troublesome about your thoughts, feelings, practices, experiences, or any other issues that are important to you about your religion. Below is a list of content features with examples. Look them over carefully and identify the most important area of distressful experiences for you. Enter the code letter on the bubble sheet and describe this area of importance in your own words on the description sheet provided.

Code (A) BELIEFS: The influence of your religious beliefs on your life

-My religious beliefs make me feel guilty

-I often feel judged by my lack of beliefs

Code (B) MEANING/DIRECTION: The implications that your beliefs on the quality of your life. -It is difficult to live up to the expectations laid down by my religion

-My parent's religion means nothing to me

Code (C) RULES AND PRACTICES: Implications that your beliefs have on governing your life -I think that certain religious restrictions are silly

-It is difficult to follow certain religious rules and practices

Code (D) NO ISSUES: This is not an issue for you

45. Go to your description sheet and record your code letter and your description of the important issues.

46. How distressful is this issue to you at this moment?

$\begin{array}{llccc}\text { Not } & \text { A little } & & \text { Very } & \text { Extremely } \\ \text { Distressful } & \text { Distressful } & \text { Distressful } & \text { Distressful } & \text { Distressful } \\ \text { A } & \text { B } & \text { C } & \text { D } & \text { E }\end{array}$

47. Have you had distress about this issue for longer than three months?
(A) Yes
(B) No

48. Have you had distress about this issue which significantly interfered with your daily living?
(A) Yes
(B) No

49. Of the following statements, which best describes your current thoughts and feelings with respect to religious issues:

(A). I am looking at different religious issues in terms of what is right for me, but I am still not sure what religion means to me.

(B). I have never really questioned my religious beliefs because I have always known what I believed.

(C). I am not sure about my religious beliefs and I have not spent much time questioning or thinking about it.

(D). I have thought a lot about religious matters and I have chosen the path that is right for me 


\section{Part $\boldsymbol{H}$ asks about moral issues. \\ PART H: SECTION I}

\section{Positive Content Features \\ (with examples)}

We would like you to identify and describe what, if anything, you consider good or positive about your thoughts, feelings, practices, experiences, or any other issues that are important to you about your morality. Below is a list of content features with examples. Look them over carefully and identify the most important area of positive experiences for you. Enter the code letter on the bubble sheet and describe this area of importance in your own words on the description sheet provided.

Code (A) BELIEFS: The influence that your moral beliefs have on your life.

-I consider myself a moral person

-My moral values make me proud

Code (B) MEANING/DIRECTION:Implications that your morality has on the quality of your life. -When I live up to my moral standards I feel fulfilled

-My moral values guide me as to what is right and wrong

Code (C) RULES AND PRACTICES: The effect that your morality has for regulating your life.

-I do not steal because it is immoral

-I always try to do the right thing

Code (D) NO ISSUES: This is not an issue for you

50. Go to your description sheet and record your code letter and your description of the important issues.

51. How positive is this issue to you at this moment?

Not

Positive

A
A little

Positive

B
Positive

C
Very

Positive

D

D
Extremely

Positive

E 


\section{PART H: SECTION II \\ Distressful Content Features \\ (with examples)}

We would like you to identify and describe what, if anything, you consider distressful or troublesome about your thoughts, feelings, practices, experiences, or any other issues that are important to you about your morality. Below is a list of content features with examples. Look them over carefully and identify the most important area of distressful experiences for you. Enter the code letter on the bubble sheet and describe this area of importance in your own words on the description sheet provided.

Code (A) BELIEFS: The influence that your moral beliefs have on your life.

-Sometimes I do things that go against my moral beliefs

-I am uncertain about my moral beliefs

Code (B)MEANING/DIRECTION:Implications that your morality has on the quality of your life. -It is difficult to guide my life consistent with my moral standards.

-I am not sure about the role morality should play in my life.

Code (C) RULES AND PRACTICES: The effect that your morality has for regulating your life. -It is difficult to live with people who do not have similar moral standards as me -It is difficult to live up to my moral rules

Code (D) NO ISSUES: This is not an issue for you

52. Go to your description sheet and record your code letter and your description of the important issues.

53. How distressful is this issue to you at this moment?

$\begin{array}{clccc}\text { Not } & \text { A little } & & \text { Very } & \text { Extremely } \\ \text { Distressful } & \text { Distressful } & \text { Distressful } & \text { Distressful } & \text { Distressful } \\ \text { A } & \text { B } & \text { C } & \text { D } & \text { E }\end{array}$

54. Have you had distress about this issue for longer than three months?
(A) Yes
(B) No

55. Have you had distress about this issue which significantly interfered with your daily living?
(A) Yes
(B) No

56. Of the following statements, which best describes your current thoughts and feelings with respect to moral issues:

(A). I find myself discussing and evaluating my moral issues and values but I have not yet found an acceptable viewpoint.

(B). I am not sure about my moral values and I have not spent much time questioning or thinking about it.

(C). After a lot of self-examination and experience, I have established a clear sense of the moral guidelines in which to live my life.

(D). I have never really questioned my moral values because I have always known what I believed to be right and wrong. 


\section{Part I asks about political issues. PART I: SECTION I}

\section{Positive Content Features \\ (with examples)}

We would like you to identify and describe what, if anything, you consider good or positive about your thoughts, feelings, practices, experiences, or any other issues that are important to you about your political issues. Below is a list of content features with examples. Look them over carefully and identify the most important area of positive experiences for you. Enter the code letter on the bubble sheet and describe this area of importance in your own words on the description sheet provided.

Code (A) BELIEFS: The influence that your political beliefs have on your life.

-my political beliefs make me feel proud

-I am open to different viewpoints

Code (B)MEANING/DIRECTION:Implications that your political beliefs has on the quality of your life.

-I feel my political beliefs help me to guide my life

-I always try to vote in accordance with my political views

Code (C) RULES AND PRACTICES: The effect that your political beliefs has for regulating your life.

-I feel privledged that I am able to vote

-I like the way my political leaders represent and interpret the law

Code (D) NO ISSUES: This is not an issue for you

57. Go to your description sheet and record your code letter and your description of the important issues.

58. How positive is this issue to you at this moment?

$\begin{array}{lllll}\text { Not } & \text { A little } & & \text { Very } & \text { Extremely } \\ \text { Positive } & \text { Positive } & \text { Positive } & \text { Positive } & \text { Positive }\end{array}$
A
B
$\mathrm{C}$
D
E 


\section{PART I: SECTION II \\ Distressful Content Features \\ (with examples)}

We would like you to identify and describe what, if anything, you consider distressful or troublesome about your thoughts, feelings, practices, experiences, or any other issues that are important to you about your political issues. Below is a list of content features with examples. Look them over carefully and identify the most important area of distressful experiences for you. Enter the code letter on the bubble sheet and describe this area of importance in your own words on the description sheet provided.

Code (A)_BELIEFS: The influence that your political beliefs have on your life.

-I am confused about my ideas on politics

-I am not sure what political platform I believe in

Code (B) MEANING/DIRECTION:Implications that your political beliefs has on the quality of your life.

-It is difficult to guide my life through my political beliefs

-I am not sure how my political orientation will fit into my family structure

Code (C) RULES AND PRACTICES: The effect that your political beliefs has for regulating your life.

-Some political decisions, such as war and governmental control, upset me -It upsets me when politicians take advantage of their power and manipulate the law

Code (D) NO ISSUES: This is not an issue for you

59. Go to your description sheet and record your code letter and your description of the important issues.

60. How distressful is this issue to you at this moment?

$\begin{array}{clccc}\text { Not } & \text { A little } & & \text { Very } & \text { Extremely } \\ \text { Distressful } & \text { Distressful } & \text { Distressful } & \text { Distressful } & \text { Distressful } \\ \text { A } & \text { B } & \text { C } & \text { D } & \text { E }\end{array}$

61. Have you had distress about this issue for longer than three months?
(A) Yes
(B) No

62. Have you had distress about this issue which significantly interfered with your daily living?
(A) Yes
(B) No

$* * * * * * * * * * * * * * * * * * * * * * * * * * * * * * * * * * * * * * * * * * * * * * * * * * * * * * * * * * * * * * * * * * * * * * * * * * * * * * * * * * * * * * * *$

63. Of the following statements, which best describes your current thoughts and feelings with respect to political issues:

(A). I am not sure about my political orientation and I have not spent much time questioning or thinking about it.

(B). I find myself discussing and evaluating my political ideas but I have not yet found an acceptable viewpoint.

(C). I have never really questioned my political beliefs because I have always known what I believed to ybe right and wrong.

(D). After a lot of self-examination and experience, I have established a clear sense of the political guidelines in which to live my life. 
APPENDIX B

145 


\section{Social Institutions Rating}

Ervin Briones William Kurtines

Florida International University

Draft 


\section{SOCIAL INSTITUTIONS RATING}

Directions: This survey asks questions about social institutions in your society. Some of the social institutions are the family, the government, gender roles, religion, mass communication, educational, legal, economic, and political institutions among others. This survey asks about your satisfaction with these institutions, how much change they are undergoing, how satisfied you are with the change, how much the change affects your life, and how much you participate in the changes that are taking place.

Please write your answers on both the bubble sheet and the description sheet provided. DO NOT WRITE ON THIS QUESTIONNAIRE. Please answer every question. 


\section{BIOGRAPHICAL DATA}

Please record and bubble your answers on the Information Section of your Computerized Answer Sheet (with a \#2 Penci1):

Sex: Male Female

College: $\quad 1=$ Freshman

2 = Sophomore

3 = Junior

4 = Senior

5 = Graduate

Age: (Record and bubble your age under column "YR" on the Computerized Answer Sheet)

Participant Number: (Record and bubble your participant number under column

"IDENTIFICATION NUMBER" on the Computerized Answer Sheet)

K) Ethnicity: (Indicate your ethnicity under column " $\underline{\mathrm{K}}$ " of the SPECIAL CODES section on the Computerized Answer Sheet)

$$
\begin{aligned}
& 0=\text { Black } \\
& 1=\text { Hispanic } \\
& 2=\text { White } \\
& 3=\text { Asian } \\
& 4=\text { Native } \\
& 5=\text { Other }
\end{aligned}
$$

L) Marital Status: (Indicate your marital status under column "L" of the SPECIAL CODES section on the Computerized Answer Sheet)

$$
\begin{aligned}
& 0=\text { Single } \\
& 1=\text { Married } \\
& 2=\text { Divorced } \\
& 3=\text { Widowed }
\end{aligned}
$$

M) Socioeconomic Status: Please choose and then bubble the number from 1 (Nonprivileged) to 7 (Very privileged) that most closely represents your socioeconomic status under column "M" of the SPECIAL CODES SECTION on the Computerized Answer Sheet:

Non-privileged...................................................... Privileged

$\begin{array}{llllllll}1 & 2 & 3 & 4 & 5 & 6 & 7\end{array}$


Use the following categories (from 1 to 8 ) to answer questions $N$ ) and 0 ):

$1=$ Higher executive, proprietor of large concern (value over $\$ 100,000$ ), major professional (e.g., accountant C.P.A., architect, lawyer, physician, college teacher, etc.), military (with rank of major and above).>

$2=$ Business manager, proprietor of medium sized business (value $\$ 35,000$ $\$ 100,000$ ). lesser professional (e.g., accountant not C.P.A, chiropractor, correction officer, librarian, musician, nurse), military (Lts.. captains), teachers (elementary and high).

$3=$ Administrative Personnel, sma11 independent business $(\$ 6,000-\$ 35,000)$, and minor professional (e.g., actor, artist, clergymen, lab assistant, photographer, surveyors, etc.). farm owner $(\$ 25,000-35,000)$.

$4=$ Clerical and sale work (e.g. bank clerk, tellers, store clerk), technicians (camp counselor, dental tech., inspector) and owners of little businesses (under $\$ 6,000)$, farm owner $(10,000-\$ 20,000)$.

5 = Skilled manual employee (e.g., typewriter, baker, carpenter, electrician, hair stylist, locksmith, trained mechanic, painter, policemen, postmen, etc.), small farmer (under $\$ 10,000$ ).

6 = Machine Operator and semi-skilled employees (e.g., hospital aides, bartender. bus driver, guard, taxi driver, welder, etc.)

7 = Unskilled employee (e.g., domestics, farm helpers, unspecified hospital worker, janitor, factory worker, etc.).

$8=$ Never worked

N) Please choose and then bubble the number (from 1 to 8 ) that most closely represents your own occupational category, under column "N" of the SPECIAL CODES SECTION on the Computerized Answer Sheet:

0) Please choose and then bubble the number (from 1 to 8 ) that most closely represents the occupational category of your parent or guardian (head of household) under column "0" of the SPECIAL CODES SECTION on the Computerized Answer Sheet.

P) Please choose and then bubble the Educational Category of your parent or guardian (head of household) under column "0" on the computerized answer sheet:

$1=$ Graduate Professional Training.

$2=$ Standard College Graduation (completed four year-college course leading to a recognized college degree).

$3=$ Partial College Training (completed at least one year college course but did not graduate).

$4=$ High School Graduates

$5=$ Partial High School (completed the tenth or the eleventh grades but did not graduate).

$6=$ Junior High School (completed the seventh grade through the ninth).

$7=$ Less than 7 years of school. 


\section{GOVERNMENT}

(Please, bubble your answers on the Computerized Answer Sheet starting with \#1:)

1. Are you satisfied with the government (as a social institution) as it is today in your society?
(A) Yes
(B) No
(C) No Opinion
(D) Uncertain

2. How satisfied are you with the government as it is today in your society?

$\begin{array}{ccccc}\text { Not } & \text { A Little } & & \text { Very } & \text { Extremely } \\ \text { Satisfied } & \text { Satisfied } & \text { Satisfied } & \text { Satisfied } & \text { Satisfied } \\ \text { A } & \text { B } & \text { C } & \text { D } & \text { E }\end{array}$

3. How unsatisfied are you with the government as it is today in your society?
Not
A Little
Very
Extremely

Unsatisfied Unsatisfied Unsatisfied Unsatisfied Unsatisfied A

B

C

D

4. To what extent is the government undergoing change in your society?

Not

Undergoing

Change

A Undergoing A Little Change

B
Undergoing

Moderate

Change

C
Undergoing

$A$ Lot of

Change

D
Undergoing

Extreme

Change

5. How much of a positive effect does this change have on the quality of your life?

$\begin{array}{ccccc}\text { Not } & \text { A Little } & & \text { Very } & \text { Extremely } \\ \text { Positive } & \text { Positive } & \text { Positive } & \text { Positive } & \text { Positive } \\ \text { A } & \text { B } & \text { C } & \text { D } & \text { E }\end{array}$

6. How much of a negative effect does this change have on the quality of your life?

$\begin{array}{ccccc}\text { Not } & \text { A Little } & & \text { Very } & \text { Extremely } \\ \text { Negative } & \text { Negative } & \text { Negative } & \text { Negative } & \text { Negative } \\ \text { A } & B & \text { C } & \text { D } & \text { E }\end{array}$

7. To what extent are you participating in this change?

$$
\text { No Slight Some A Lot of Maximum }
$$

Participation Participation Participation Participation Participation

A

B

C

D 
8. To what extent are other people participating in this change?

$$
\text { No Slight Some A Lot of Maximum }
$$

Participation Participation Participation Participation Participation

A

B

C

D

$\mathrm{E}$

9. To what extent is the government in your society open to influences?

$\begin{array}{lcccc}\text { Not } & \text { A Little } & & \text { Very } & \text { Extremely } \\ \text { Open } & \text { Open } & \text { Open } & \text { Open } & \text { Open } \\ \text { A } & \text { B } & \text { C } & \text { D } & \text { E }\end{array}$

10. How much of a positive effect does this openness (or lack of openness) have on the quality of your life?

$\begin{array}{ccccc}\text { Not } & \text { A Little } & & \text { Very } & \text { Extremely } \\ \text { Positive } & \text { Positive } & \text { Positive } & \text { Positive } & \text { Positive } \\ \text { A } & \text { B } & \text { C } & \text { D } & \text { E }\end{array}$

11. How much of a negative effect does this openness (or lack of openness) have on the quality or your life?

$\begin{array}{ccccc}\text { Not } & \text { A Little } & & \text { Very } & \text { Extremely } \\ \text { Negative } & \text { Negative } & \text { Negative } & \text { Negative } & \text { Negative } \\ \text { A } & \text { B } & \text { C } & \text { D } & \text { E }\end{array}$

12. To what extent is the government in your society accessible to you? (how feasible is it for you as a citizen to have access to, to make use of, or to contact this institution)
Not
A Little
Very
Extremely
Accessible
Accessible
Accessible
A
B
c
Accessible Accessible

13. How much of a positive effect does this accessibility (or lack of accessibility) have on the quality of your life?

$\begin{array}{ccccc}\text { Not } & \text { A Little } & & \text { Very } & \text { Extremely } \\ \text { Positive } & \text { Positive } & \text { Positive } & \text { Positive } & \text { Positive } \\ \text { A } & \text { B } & \text { C } & \text { D } & \text { E }\end{array}$

14. How much of a negative effect does this accessibility (or lack of accessibility) have on the quality or your life?

Not

Negative

A
A Little

Negative

$B$
Very

Negative

D
Extremely

Negative 
15. To what extent is the government in your society supportive to you? (How much does it serve your interests and needs or help to facilitate your goals)

$\begin{array}{ccccc}\text { Not } & \text { A Little } & & \text { Very } & \text { Extremely } \\ \text { Supportive } & \text { Supportive } & \text { Supportive } & \text { Supportive } & \text { Supportive } \\ \text { A } & \text { B } & \text { C } & \text { D } & \text { E }\end{array}$

16. How much of a positive effect does this support (or lack of support) have on the quality of your life?

$\begin{array}{ccccc}\text { Not } & \text { A Little } & & \text { Very } & \text { Extremely } \\ \text { Positive } & \text { Positive } & \text { Positive } & \text { Positive } & \text { Positive } \\ \text { A } & \text { B } & \text { C } & \text { D } & \text { E }\end{array}$

17. How much of a negative effect does this support (or lack of support) have on the quality or your life?

$\begin{array}{ccccc}\text { Not } & \text { A Little } & & \text { Very } & \text { Extremely } \\ \text { Negative } & \text { Negative } & \text { Negative } & \text { Negative } & \text { Negative } \\ \text { A } & \text { B } & \text { C } & \text { D } & \text { E }\end{array}$

17a. We would like you to describe what, if anything, you consider good or positive about the government in your society in terms of your thoughts, feelings, practices, experiences, or any other issues that are important to you. Please, describe in your own words on the DESCRIPTION SHEET provided:

18. How positive is this issue in your life?

$\begin{array}{ccccc}\text { Slightly } & \text { A Little } & & \text { Very } & \text { Extremely } \\ \text { Positive } & \text { Positive } & \text { Positive } & \text { Positive } & \text { Positive } \\ \text { A } & \text { B } & \text { C } & \text { D } & \text { E }\end{array}$

18a. We would like you to describe what, if anything, you consider distressful or troublesome about the government in your society in terms of your thoughts, feelings, practices, experiences, or any other issues that are important to you. Please, describe in your own words on the DESCRIPTION SHEET provided:

19. How distressful is this issue in your life?

Slightly A Little Very Extremely Distressful Distressful Distressful Distressful Distressful A 


\section{ECONOMIC INSTITUTIONS}

(Examples of Economic Institutions are small private businesses, credit card companies, investment firms, banks, financing companies, large companies, advertising agencies, etc.)

20. Are you satisfied with the economic institutions as they are today in your society?
(A) Yes
(B) No
(C) No Opinion
(D) Uncertain

21. How satisfied are you with the economic institutions as they are today in your society?

\begin{tabular}{|c|c|c|}
\hline $\begin{array}{l}\text { Not } \\
\text { tisfied }\end{array}$ & $\begin{array}{l}\text { A Little } \\
\text { Satisfied }\end{array}$ & \\
\hline
\end{tabular}
A
$\mathrm{B}$
C
D
E

22. How unsatisfied are you with the economic institutions as they are today in your society?
Not
A Little
Very
Extremely

Unsatisfied Unsatisfied Unsatisfied Unsatisfied Unsatisfied A B C D

E

23. To what extent are the economic institutions undergoing change in your society?

$\begin{array}{ccccc}\text { Not } & \text { Undergoing } & \text { Undergoing } & \text { Undergoing } & \text { Undergoing } \\ \text { Undergoing } & \text { A Little } & \text { Moderate } & \text { A Lot of } & \text { Extreme } \\ \text { Change } & \text { Change } & \text { Change } & \text { Change } & \text { Change } \\ \text { A } & \text { B } & \text { C } & \text { D } & E\end{array}$

24. How much of a positive effect does this change have on the quality of your life?

$\begin{array}{ccccc}\text { Not } & \text { A Little } & & \text { Very } & \text { Extremely } \\ \text { Positive } & \text { Positive } & \text { Positive } & \text { Positive } & \text { Positive } \\ \text { A } & \text { B } & \text { C } & \text { D } & \text { E }\end{array}$

25. How much of a negative effect does this change have on the quality or your life?

Not

Negative

A

A Little

Very

Extremely

Negative

Negative

C

Negative

Negative

B

D

E 
26. To what extent are you participating in this change?

No Slight Some A Lot of Maximum

Participation Participation Participation Participation Participation
A
B
C
D E

27. To what extent are other people participating in this change?
No
Slight
Some
A Lot of
Maximum

Participation Participation Participation Participation Participation
A
B
C
E

28. To what extent are the economic institutions in your society open to influences?

$\begin{array}{lcccc}\text { Not } & \text { A Little } & & \text { Very } & \text { Extremely } \\ \text { Open } & \text { Open } & \text { Open } & \text { Open } & \text { Open } \\ \text { A } & \text { B } & \text { C } & \text { D } & \text { E }\end{array}$

29. How much of a positive effect does this openness (or lack of openness) have on the quality of your life?

$\begin{array}{ccccc}\text { Not } & \text { A Little } & & \text { Very } & \text { Extremely } \\ \text { Positive } & \text { Positive } & \text { Positive } & \text { Positive } & \text { Positive } \\ \text { A } & \text { B } & \text { C } & \text { D } & \text { E }\end{array}$

30. How much of a negative effect does this openness (or lack of openness) have on the quality or your life?

$\begin{array}{ccccc}\text { Not } & \text { A Little } & & \text { Very } & \text { Extremely } \\ \text { Negative } & \text { Negative } & \text { Negative } & \text { Negative } & \text { Negative } \\ \text { A } & \text { B } & \text { C } & \text { D } & \text { E }\end{array}$

31. To what extent are the economic institutions in your society accessible to you? (how feasible is it for you as a citizen to have access to, to make use of, or to contact these institutions)

$\begin{array}{ccccc}\text { Not } & \text { A Little } & & \text { Very } & \text { Extremely } \\ \text { Accessible } & \text { Accessible } & \text { Accessible } & \text { Accessible } & \text { Accessible } \\ \text { A } & \text { B } & \text { C } & \text { D } & \text { E }\end{array}$

32. How much of a positive effect does this accessibility (or lack of accessibility) have on the quality of your life?

$\begin{array}{ccccc}\text { Not } & \text { A Little } & & \text { Very } & \text { Extremely } \\ \text { Positive } & \text { Positive } & \text { Positive } & \text { Positive } & \text { Positive } \\ \text { A } & \text { B } & \text { C } & \text { D } & \text { E }\end{array}$


33. How much of a negative effect does this accessibility (or lack of accessibility) have on the quality or your life?

$\begin{array}{ccccc}\text { Not } & \text { A Little } & & \text { Very } & \text { Extremely } \\ \text { Negative } & \text { Negative } & \text { Negative } & \text { Negative } & \text { Negative } \\ \text { A } & \text { B } & \text { C } & \text { D } & \text { E }\end{array}$

34. To what extent are the economic institutions in your society supportive to you? (how much do they serve your interests and needs or help to facilitate your goals)

$\begin{array}{ccccc}\text { Not } & \text { A Little } & & \text { Very } & \text { Extremely } \\ \text { Supportive } & \text { Supportive } & \text { Supportive } & \text { Supportive } & \text { Supportive } \\ \text { A } & \text { B } & \text { C } & \text { D } & \text { E }\end{array}$

35. How much of a positive effect does this support (or lack of support) have on the quality of your life?

$\begin{array}{ccccc}\text { Not } & \text { A Little } & & \text { Very } & \text { Extremely } \\ \text { Positive } & \text { Positive } & \text { Positive } & \text { Positive } & \text { Positive } \\ \text { A } & \text { B } & \text { C } & \text { D } & \text { E }\end{array}$

36. How much of a negative effect does this support (or 1ack of support) have on the quality or your life?

$\begin{array}{ccccc}\text { Not } & \text { A Little } & & \text { Very } & \text { Extremely } \\ \text { Negative } & \text { Negative } & \text { Negative } & \text { Negative } & \text { Negative } \\ \text { A } & B & \text { C } & \text { D } & \text { E }\end{array}$

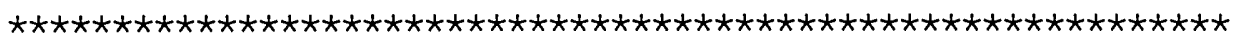

36a. We would like you to describe what, if anything, you consider good or positive about the economic institutions in your society in terms of your thoughts, feelings, practices, experiences, or any other issues that are important to you. Please, describe in your own words on the DESCRIPTION SHEET provided:

37. How positive is this issue in your life? (Please, circle one number: )

$\begin{array}{ccccc}\text { Sightiy } & \text { A Little } & & \text { Very } & \text { Extremely } \\ \text { Positive } & \text { Positive } & \text { Positive } & \text { Positive } & \text { Positive } \\ \text { A } & \text { B } & \text { C } & \text { D } & \text { E }\end{array}$


37a. We would 1ike you to describe what, if anything, you consider distressful or troublesome about the economic institutions in your society in terms of your thoughts, feelings, practices, experiences, or any other issues that are important to you. Please, describe in your own words on the DESCRIPTION SHEET provided:

38. How distressful is this issue in your life?

$\begin{array}{ccccc}\text { Slightly } & \text { A Little } & & \text { Very } & \text { Extremely } \\ \text { Distressful } & \text { Distressful } & \text { Distressful } & \text { Distressful } & \text { Distressful } \\ \text { A } & \text { B } & \text { C } & \text { D } & \text { E }\end{array}$




\section{EDUCATIONAL INSTITUTIONS}

(Examples of Educational Institutions are pre-schools, elementary and secondary schools, technical schools, academies, institutes. colleges, universities, etc.)

39. Are you satisfied with the educational institutions as they are today in your society?
(A) Yes
(B) No
(C) No Opinion
(D) Uncertain

40. How satisfied are you with the educational institutions as they are today in your society?

$\begin{array}{ccccc}\text { Not } & \text { A Little } & & \text { Very } & \text { Extremely } \\ \text { Satisfied } & \text { Satisfied } & \text { Satisfied } & \text { Satisfied } & \text { Satisfied } \\ \text { A } & \text { B } & \text { C } & \text { D } & \text { E }\end{array}$

41. How unsatisfied are you with the educational institutions as they are today in your society?

Not

A Little

Very

Extremely

Unsatisfied Unsatisfied Unsatisfied Unsatisfied Unsatisfied

A

B

c

D

$\mathrm{E}$

42. To what extent are the educational institutions undergoing change in your society?

$\begin{array}{ccccc}\text { Not } & \text { Undergoing } & \text { Undergoing } & \text { Undergoing } & \text { Undergoing } \\ \text { Undergoing } & \text { A Little } & \text { Moderate } & \text { A Lot of } & \text { Extreme } \\ \text { Change } & \text { Change } & \text { Change } & \text { Change } & \text { Change } \\ \text { A } & \text { B } & \text { C } & \text { D } & E\end{array}$

43. How much of a positive effect does this change have on the quality of your $1 \mathrm{ife}$ ?

$\begin{array}{ccccc}\text { Not } & \text { A Little } & & \text { Very } & \text { Extremely } \\ \text { Positive } & \text { Positive } & \text { Positive } & \text { Positive } & \text { Positive } \\ \text { A } & \text { B } & \text { C } & \text { D } & \text { E }\end{array}$

44. How much of a negative effect does this change have on the quality or your life?

Not Negative

A

A Little

Negative

B
Very

Negative

c
Negative

D
Extremely

Negative

E 
45. To what extent are you participating in this change?

No Slight Some A Lot of Maximum

Participation Participation Participation Participation Participation A

46. To what extent are other people participating in this change?

No Slight Some A Lot of Maximum

Participation Participation Participation Participation Participation
A
B
C
D
E

47. To what extent are the educational institutions in your society open to influences?

$\begin{array}{lcccc}\text { Not } & \text { A Little } & & \text { Very } & \text { Extremely } \\ \text { Open } & \text { Open } & \text { Open } & \text { Open } & \text { Open } \\ \text { A } & \text { B } & \text { C } & \text { D } & \text { E }\end{array}$

48. How much of a positive effect does this openness (or lack of openness) have on the quality of your life?

$\begin{array}{ccccc}\text { Not } & \text { A Little } & & \text { Very } & \text { Extremely } \\ \text { Positive } & \text { Positive } & \text { Positive } & \text { Positive } & \text { Positive } \\ \text { A } & \text { B } & \text { C } & \text { D } & \text { E }\end{array}$

49. How much of a negative effect does this openness (or lack of openness) have on the quality or your life?

$\begin{array}{ccccc}\text { Not } & \text { A Little } & & \text { Very } & \text { Extremely } \\ \text { Negative } & \text { Negative } & \text { Negative } & \text { Negative } & \text { Negative } \\ \text { A } & B & \text { C } & \text { D } & \text { E }\end{array}$

50. To what extent are the educational institutions in your society accessible to you? (how feasible is it for you as a citizen to have access to, to make use of, or to contact these institutions)

$\begin{array}{ccccc}\text { Not } & \text { A Little } & \text { Very } & \text { Extremely } \\ \text { Accessible } & \text { Accessible } & \text { Accessible } & \text { Accessible } & \text { Accessible } \\ \text { A } & \text { B } & \text { C } & \text { D } & \text { E }\end{array}$

51. How much of a positive effect does this accessibility (or lack of accessibility) have on the quality of your life?

Not

Positive

A
A Little

Positive

B
Positive

c
Very

Positive

D
Extremely

Positive

E 
52. How much of a negative effect does this accessibility (or lack of accessibility) have on the quality or your life?

$\begin{array}{ccccc}\text { Not } & \text { A Little } & & \text { Very } & \text { Extremely } \\ \text { Negative } & \text { Negative } & \text { Negative } & \text { Negative } & \text { Negative } \\ \text { A } & \text { B } & \text { C } & \text { D } & \text { E }\end{array}$

53. To what extent are the educational institutions in your society supportive to you? (how much do they serve your interests and needs or help to facilitate your goals)

Not A Little Very Extremely Supportive Supportive Supportive Supportive Supportive $\begin{array}{lllll}A & B & C & D & E\end{array}$

54. How much of a positive effect does this support (or lack of support) have on the quality of your life?

$\begin{array}{ccccc}\text { Not } & \text { A Little } & & \text { Very } & \text { Extremely } \\ \text { Positive } & \text { Positive } & \text { Positive } & \text { Positive } & \text { Positive } \\ \text { A } & \text { B } & \text { C } & \text { D } & \text { E }\end{array}$

55. How much of a negative effect does this support (or lack of support) have on the quality or your life?

$\begin{array}{ccccc}\text { Not } & \text { A Little } & & \text { Very } & \text { Extremely } \\ \text { Negative } & \text { Negative } & \text { Negative } & \text { Negative } & \text { Negative } \\ \text { A } & \text { B } & \text { C } & \text { D } & \text { E }\end{array}$

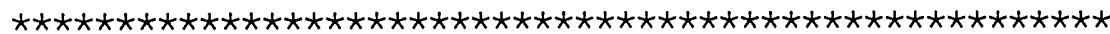

55a. We would like you to describe what, if anything, you consider good or positive about the educational institutions in your society in terms of your thoughts, feelings, practices, experiences, or any other issues that are important to you. Please, describe in your own words on the DESCRIPTION SHEET provided:

56. How positive is this issue in your 1 ife? (Please, circle one number: )

$\begin{array}{ccccc}\text { Slightly } & \text { A Little } & & \text { Very } & \text { Extremely } \\ \text { Positive } & \text { Positive } & \text { Positive } & \text { Positive } & \text { Positive } \\ \text { A } & \text { B } & \text { C } & \text { D } & \text { E }\end{array}$


56a. We would like you to describe what, if anything, you consider distressful or troublesome about the educational institutions in your society in terms of your thoughts, feelings, practices, experiences, or any other issues that are important to you. Please, describe in your own words on the DESCRIPTION SHEET provided:

57. How distressful is this issue in your life?

Slightly

Distressful

A
A Little Distressful

B

\section{Distressful}

C
Very

Distressful

D
Extremely

Distressful

E 


\section{LEGAL/JUDICIAL INSTITUTIONS}

(Examples of Lega7/Judicial Institutions are civil courts, criminal courts, the supreme court, house of representatives, senate, etc.)

58. Are you satisfied with the legal/judicial institutions as they are today in your society?
(A) Yes
(B) No
(C) No Opinion
(D) Uncertain

59. How satisfied are you with the legal/judicial institutions as they are today in your society?

$\begin{array}{ccccc}\text { Not } & \text { A Little } & & \text { Very } & \text { Extremely } \\ \text { Satisfied } & \text { Satisfied } & \text { Satisfied } & \text { Satisfied } & \text { Satisfied } \\ \text { A } & \text { B } & \text { C } & \text { D } & \text { E }\end{array}$

60. How unsatisfied are you with the legal/judicial institutions as they are today in your society?

Not

A Little

Very

Extremely

Unsatisfied Unsatisfied Unsatisfied Unsatisfied Unsatisfied A B C

61. To what extent are the legal/judicial institutions undergoing change in your society? Not Undergoing Undergoing Undergoing Undergoing

$\begin{array}{ccccc}\text { Undergoing } & \text { A Little } & \text { Moderate } & \text { A Lot of } & \text { Extreme } \\ \text { Change } & \text { Change } & \text { Change } & \text { Change } & \text { Change } \\ \text { A } & \text { B } & \text { C } & \text { D } & \text { E }\end{array}$

62. How much of a positive effect does this change have on the quality of your life?

$\begin{array}{ccccc}\text { Not } & \text { A Little } & & \text { Very } & \text { Extremely } \\ \text { Positive } & \text { Positive } & \text { Positive } & \text { Positive } & \text { Positive } \\ \text { A } & \text { B } & \text { C } & \text { D } & \text { E }\end{array}$

63. How much of a negative effect does this change have on the quality or your life?

Not

Negative

A
A Little

Negative

B
Negative

C
Very

Negative

D
Extremely

Negative

E 
64. To what extent are you participating in this change?

$$
\text { No Slight Some A Lot of Maximum }
$$

Participation Participation Participation Participation Participation

$$
\begin{array}{lllll}
A & B & C & \text { D } & \text { E }
\end{array}
$$

65. To what extent are other people participating in this change?

No Slight Some A Lot of Maximum

Participation Participation Participation Participation Participation

A

B

C

D

E

66. To what extent are the legal/judicial institutions in your society open to influences?

$\begin{array}{lcccc}\text { Not } & \text { A Little } & & \text { Very } & \text { Extremely } \\ \text { Open } & \text { Open } & \text { Open } & \text { Open } & \text { Open } \\ \text { A } & \text { B } & \text { C } & \text { D } & \text { E }\end{array}$

67. How much of a positive effect does this openness (or lack of openness) have on the quality of your life?

$\begin{array}{ccccc}\text { Not } & \text { A Little } & & \text { Very } & \text { Extremely } \\ \text { Positive } & \text { Positive } & \text { Positive } & \text { Positive } & \text { Positive } \\ \text { A } & \text { B } & \text { C } & \text { D } & \text { E }\end{array}$

68. How much of a negative effect does this openness (or lack of openness) have on the quality or your life?

$\begin{array}{ccccc}\text { Not } & \text { A Little } & & \text { Very } & \text { Extremely } \\ \text { Negative } & \text { Negative } & \text { Negative } & \text { Negative } & \text { Negative } \\ \text { A } & \text { B } & \text { C } & \text { D } & \text { E }\end{array}$

69. To what extent are the legal/judicial institutions in your society accessible to you? (how feasible is it for you as a citizen to have access to, to make use of, or to contact these institutions)
Not
A Little
Very
Extremely Accessible Accessible
Accessible Accessible Accessible A B c D E

70. How much of a positive effect does this accessibility (or lack of accessibility) have on the quality of your 1 ife?

Not

Positive

A
A Little

Positive

B
Positive

c
Very

Positive

D
Extremely Positive 
71. How much of a negative effect does this accessibility (or lack of accessibility) have on the quality or your life?
Not
A Little
Negative
B
Very
Extremely
A
Negative
C
Negative Negative
$E$

Negative

72. To what extent are the legal/judicial institutions in your society supportive to you? (how much do they serve your interests and needs or help to facilitate your goals)
Not
A Little
Very
Extremely

Supportive Supportive

A

B

Supportive

Supportive Supportive

C

E

73. How much of a positive effect does this support (or lack of support) have on the quality of your life?

$\begin{array}{ccccc}\text { Not } & \text { A Little } & & \text { Very } & \text { Extremely } \\ \text { Positive } & \text { Positive } & \text { Positive } & \text { Positive } & \text { Positive } \\ \text { A } & \text { B } & \text { C } & \text { D } & \text { E }\end{array}$

74. How much of a negative effect does this support (or lack of support) have on the quality or your life?

$\begin{array}{ccccc}\text { Not } & \text { A Little } & & \text { Very } & \text { Extremely } \\ \text { Negative } & \text { Negative } & \text { Negative } & \text { Negative } & \text { Negative } \\ \text { A } & \text { B } & \text { C } & \text { D } & \text { E }\end{array}$

74a. We would Tike you to describe what, if anything, you consider good or positive about the legal/judicial institutions in your society in terms of your thoughts, feelings, practices, experiences, or any other issues that are important to you. Please, describe in your own words on the DESCRIPTION SHEET provided:

75. How positive is this issue in your 1ife? (Please, circle one number: )

$\begin{array}{ccccc}\text { Slightly } & \text { A Little } & & \text { Very } & \text { Extremely } \\ \text { Positive } & \text { Positive } & \text { Positive } & \text { Positive } & \text { Positive } \\ \text { A } & \text { B } & \text { C } & \text { D } & \text { E }\end{array}$


75a. We would like you to describe what, if anything, you consider distressful or troublesome about the legal/judicial institutions in your society in terms of your thoughts, feelings, practices, experiences, or any other issues that are important to you. Please, describe in your own words on the DESCRIPTION SHEET provided:

76. How distressful is this issue in your life?

Slightly A Little Distressful Distressful

A
B
Distressful

C
Very Extremely Distressful Distressful 
POLITICAL INSTITUTIONS

(Examples of Political Institutions are the electoral college or the voting system. national parties, consumer groups, political action groups, etc.)

77. Are you satisfied with the political institutions as they are today in your society?
(A) Yes
(B) No
(C) No Opinion
(D) Uncertain

78. How satisfied are you with the political institutions as they are today in your society?

$\begin{array}{ccccc}\text { Not } & \text { A Little } & & \text { Very } & \text { Extremely } \\ \text { Satisfied } & \text { Satisfied } & \text { Satisfied } & \text { Satisfied } & \text { Satisfied } \\ \text { A } & \text { B } & \text { C } & \text { D } & \text { E }\end{array}$

79. How unsatisfied are you with the political institutions as they are today in your society?

$$
\text { Not A Little Very Extremely }
$$

Unsatisfied Unsatisfied Unsatisfied Unsatisfied Unsatisfied
A
$\mathrm{B}$
C
D
E

80. To what extent are the political institutions undergoing change in your society?

$\begin{array}{ccccc}\text { Not } & \text { Undergoing } & \text { Undergoing } & \text { Undergoing } & \text { Undergoing } \\ \text { Undergoing } & \text { A Little } & \text { Moderate } & \text { A Lot of } & \text { Extreme } \\ \text { Change } & \text { Change } & \text { Change } & \text { Change } & \text { Change } \\ \text { A } & \text { B } & \text { C } & \text { D } & \text { E }\end{array}$

81. How much of a positive effect does this change have on the quality of your life?

$\begin{array}{ccccc}\text { Not } & \text { A Little } & & \text { Very } & \text { Extremely } \\ \text { Positive } & \text { Positive } & \text { Positive } & \text { Positive } & \text { Positive } \\ \text { A } & \text { B } & \text { C } & \text { D } & \text { E }\end{array}$

82. How much of a negative effect does this change have on the quality or your 7 ife?

$\begin{array}{ccccc}\text { Not } & \text { A Little } & & \text { Very } & \text { Extremely } \\ \text { Negative } & \text { Negative } & \text { Negative } & \text { Negative } & \text { Negative } \\ \text { A } & \text { B } & \text { C } & \text { D } & \text { E }\end{array}$


83. To what extent are you participating in this change?
No
Slight
Some
A Lot of
Maximum

Participation Participation Participation Participation Participation
A
B
C
D
E

84. To what extent are other people participating in this change?
No
slight
Some
A Lot of
Maximum

Participation Participation Participation Participation Participation
A
B
C
D
E

85. To what extent are the political institutions in your society open to influences?

$\begin{array}{lcccc}\text { Not } & \text { A Little } & & \text { Very } & \text { Extremely } \\ \text { Open } & \text { Open } & \text { Open } & \text { Open } & \text { Open } \\ \text { A } & \text { B } & \text { C } & \text { D } & \text { E }\end{array}$

86. How much of a positive effect does this openness (or lack of openness) have on the quality of your life? Not A Little Very Extremely

$\begin{array}{ccccc}\text { Positive } & \text { Positive } & \text { Positive } & \text { Positive } & \text { Positive } \\ \text { A } & B & C & D & E\end{array}$

87. How much of a negative effect does this openness (or lack of openness) have on the quality or your life?

$\begin{array}{ccccc}\text { Not } & \text { A Little } & & \text { Very } & \text { Extremely } \\ \text { Negative } & \text { Negative } & \text { Negative } & \text { Negative } & \text { Negative } \\ \text { A } & \text { B } & \text { C } & \text { D } & \text { E }\end{array}$

88. To what extent are the political institutions in your society accessible to you? (how feasible is it for you as a citizen to have access to. to make use of, or to contact these institutions)

$\begin{array}{ccccc}\text { Not } & \text { A Little } & \text { Very } & \text { Extremely } \\ \text { Accessible } & \text { Accessible } & \text { Accessible } & \text { Accessible } & \text { Accessible } \\ \text { A } & \text { B } & \text { C } & \text { D } & \text { E }\end{array}$

89. How much of a positive effect does this accessibility (or lack of accessibility) have on the quality of your life?

$\begin{array}{ccccc}\text { Not } & \text { A Little } & & \text { Very } & \text { Extremely } \\ \text { Positive } & \text { Positive } & \text { Positive } & \text { Positive } & \text { Positive } \\ \text { A } & \text { B } & \text { C } & \text { D } & \text { E }\end{array}$


90. How much of a negative effect does this accessibility (or lack of accessibility) have on the quality or your life?

$\begin{array}{ccccc}\text { Not } & \text { A Little } & & \text { Very } & \text { Extremely } \\ \text { Negative } & \text { Negative } & \text { Negative } & \text { Negative } & \text { Negative } \\ \text { A } & \text { B } & \text { C } & \text { D } & \text { E }\end{array}$

91. To what extent are the political institutions in your society supportive to you? (how much do they serve your interests and needs or help to facilitate your goals)

$\begin{array}{ccccc}\text { Not } & \text { A Little } & & \text { Very } & \text { Extremely } \\ \text { Supportive } & \text { Supportive } & \text { Supportive } & \text { Supportive } & \text { Supportive } \\ \text { A } & \text { B } & \text { C } & \text { D } & \text { E }\end{array}$

92. How much of a positive effect does this support (or lack of support) have on the quality of your life?

$\begin{array}{ccccc}\text { Not } & \text { A Little } & & \text { Very } & \text { Extremely } \\ \text { Positive } & \text { Positive } & \text { Positive } & \text { Positive } & \text { Positive } \\ \text { A } & \text { B } & \text { C } & \text { D } & \text { E }\end{array}$

93. How much of a negative effect does this support (or lack of support) have on the quality or your life?

$\begin{array}{ccccc}\text { Not } & \text { A Little } & & \text { Very } & \text { Extremely } \\ \text { Negative } & \text { Negative } & \text { Negative } & \text { Negative } & \text { Negative } \\ \text { A } & \text { B } & \text { C } & \text { D } & \text { E }\end{array}$

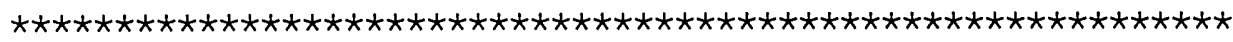

93a. We would like you to describe what, if anything, you consider good or positive about the political institutions in your society in terms of your thoughts, feelings, practices, experiences, or any other issues that are important to you. Please, describe in your own words on the DESCRIPTION SHEET provided:

94. How positive is this issue in your life? (Please, circle one number: )

$\begin{array}{cc}\text { Slightly } & \text { A Little } \\ \text { Positive } & \text { Positive } \\ \text { A } & \text { B }\end{array}$

$\begin{array}{ccc} & \text { Very } & \text { Extremely } \\ \text { Positive } & \text { Positive } & \text { Positive } \\ \text { C } & \text { D } & \text { E }\end{array}$


94a. We would like you to describe what, if anything, you consider distressful or troublesome about the political institutions in your society in terms of your thoughts, feelings, practices, experiences, or any other issues that are important to you. Please, describe in your own words on the DESCRIPTION SHEET provided:

95. How distressful is this issue in your $7 \mathrm{ife}$ ?

Slightly A Little

Distressful Distressful

A
B
Distressful

C
Very

DistressfuT

D
Extremely

Distressful 


\section{MASS COMMUNICATION}

(Examples of mass communication institutions are the radio, television. newspapers, computerized media, mass mailing, etc.)

96. Are you satisfied with the mass communication (as a social institution) as it is today in your society?
(A) Yes
(B) No
(C) No Opinion
(D) Uncertain

97. How satisfied are you with the mass communication as it is today in your society?

Not A Little Very Extremely

Satisfied Satisfied Satisfied Satisfied Satisfied $\begin{array}{lllll}A & B & C & D & E\end{array}$

98. How unsatisfied are you with the mass communication as it is today in your society?

Not A Little Very Extremely Unsatisfied Unsatisfied Unsatisfied Unsatisfied Unsatisfied $\begin{array}{lllll}A & B & C & D & E\end{array}$

99. To what extent is the mass communication undergoing change in your society?

Not Undergoing Undergoing Undergoing Undergoing

$\begin{array}{ccccr}\text { Undergoing } & \text { A Little } & \text { Moderate } & \text { A Lot of } & \text { Extreme } \\ \text { Change } & \text { Change } & \text { Change } & \text { Change } & \text { Change } \\ \text { A } & B & \text { C } & D & \text { E }\end{array}$

100. How much of a positive effect does this change have on the quality of your life?

$\begin{array}{ccccc}\text { Not } & \text { A Little } & & \text { Very } & \text { Extremely } \\ \text { Positive } & \text { Positive } & \text { Positive } & \text { Positive } & \text { Positive } \\ \text { A } & \text { B } & \text { C } & \text { D } & \text { E }\end{array}$

101. How much of a negative effect does this change have on the quality or your life?

$\begin{array}{ccccc}\text { Not } & \text { A Little } & & \text { Very } & \text { Extremely } \\ \text { Negative } & \text { Negative } & \text { Negative } & \text { Negative } & \text { Negative } \\ \text { A } & \text { B } & \text { C } & \text { D } & \text { E }\end{array}$


102. To what extent are you participating in this change?
No
Slight
Some
A Lot of
Maximum

Participation Participation Participation Participation Participation A B C D

103. To what extent are other people participating in this change?
No
slight
some
A Lot of
Maximum

Participation Participation Participation Participation Participation A

B

c

104. To what extent is the mass communication in your society open to influences?

$\begin{array}{lcccc}\text { Not } & \text { A Little } & & \text { Very } & \text { Extremely } \\ \text { Open } & \text { Open } & \text { Open } & \text { Open } & \text { Open } \\ \text { A } & \text { B } & \text { C } & D & \text { E }\end{array}$

105. How much of a positive effect does this openness (or lack of openness) have on the quality of your 1 ife?

Not A Little Very Extremely

Positive

Positive

Positive

Positive

Positive

A

B

c

D

E

106. How much of a negative effect does this openness (or lack of openness) have on the quality or your life?

Not A Little Very Extremely

Negative Negative Negative

Negative Negative

A

B

C

D

$\mathrm{E}$

107. To what extent is the mass communication in your society accessible to you? (how feasible is it for you as a citizen to have access to. to make use of, or to contact this institution)
Not
A Little
Very
Extremely
Accessible Accessible
A
$\mathrm{B}$
Accessible
C
Accessible Accessible

108. How much of a positive effect does this accessibility (or lack of accessibility) have on the quality of your life?

$\begin{array}{ccccc}\text { Not } & \text { A Little } & & \text { Very } & \text { Extremely } \\ \text { Positive } & \text { Positive } & \text { Positive } & \text { Positive } & \text { Positive } \\ \text { A } & \text { B } & \text { C } & \text { D } & \text { E }\end{array}$


109. How much of a negative effect does this accessibility (or lack of accessibility) have on the quality or your life?

Extremely

$\begin{array}{ccccc}\text { Not } & \text { A Little } & & \text { Very } & \text { Extremely } \\ \text { Negative } & \text { Negative } & \text { Negative } & \text { Negative } & \text { Negative } \\ \text { A } & \text { B } & \text { C } & \text { D } & \text { E }\end{array}$

110. To what extent is the mass communication in your society supportive to you? (how much does it serve your interests and needs or help to facilitate your goals)

$\begin{array}{ccccc}\text { Not } & \text { A Little } & & \text { Very } & \text { Extremely } \\ \text { Supportive } & \text { Supportive } & \text { Supportive } & \text { Supportive } & \text { Supportive } \\ \text { A } & \text { B } & \text { C } & \text { D } & \text { E }\end{array}$

111. How much of a positive effect does this support (or lack of support) have on the quality of your life?

$\begin{array}{ccccc}\text { Not } & \text { A Little } & & \text { Very } & \text { Extremely } \\ \text { Positive } & \text { Positive } & \text { Positive } & \text { Positive } & \text { Positive } \\ \text { A } & \text { B } & \text { C } & \text { D } & \text { E }\end{array}$

112. How much of a negative effect does this support (or lack of support) have on the quality or your 1ife?

$\begin{array}{ccccc}\text { Not } & \text { A Little } & & \text { Very } & \text { Extremely } \\ \text { Negative } & \text { Negative } & \text { Negative } & \text { Negative } & \text { Negative } \\ \text { A } & \text { B } & \text { C } & \text { D } & \text { E }\end{array}$

112a. We would like you to describe what, if anything, you consider good or positive about the mass communication in your society in terms of your thoughts, feelings, practices, experiences, or any other issues that are important to you. Please, describe in your own words on the DESCRIPTION SHEET provided:

113. How positive is this issue in your life? (Please, circle one number: )

$\begin{array}{ccccc}\text { Slightly } & \text { A Little } & & \text { Very } & \text { Extremely } \\ \text { Positive } & \text { Positive } & \text { Positive } & \text { Positive } & \text { Positive } \\ \text { A } & \text { B } & \text { C } & \text { D } & \text { E }\end{array}$


113a. We would like you to describe what, if anything, you consider distressful or troublesome about the mass communication in your society in terms of your thoughts, feelings, practices, experiences, or any other issues that are important to you. Please, describe in your own words on the DESCRIPTION SHEET provided:

114. How distressful is this issue in your life?

$\begin{array}{ccccc}\text { Slightly } & \text { A Little } & & \text { Very } & \text { Extremely } \\ \text { Distressful } & \text { Distressful } & \text { Distressful } & \text { Distressful } & \text { Distressful } \\ \text { A } & \text { B } & \text { C } & \text { D } & \text { E }\end{array}$




\section{RELIGION}

(Examples of religions are: Christianity (Catholicism.

Protestantism, etc.), Judaism, Buddhism. Hinduism, etc.)

115. Do you identify with a particular religion in your society?
(A) Yes
(B) No
(C) Uncertain

116. Are you satisfied with your religion as it is today in your society? (If you do not identify with a particular religion, are you satisfied with religion as a social institution?)
(A) Yes
(B) No
(C) No Opinion
(D) Uncertain

117. How satisfied are you with your religion (or religion as a social institution) as it is today in your society? (please, circle one number: )

$\begin{array}{ccccc}\text { Not } & \text { A Little } & & \text { Very } & \text { Extremely } \\ \text { Satisfied } & \text { Satisfied } & \text { Satisfied } & \text { Satisfied } & \text { Satisfied } \\ \text { A } & \text { B } & \text { C } & \text { D } & \text { E }\end{array}$

118. How unsatisfied are you with your religion (or religion as a social institution) as it is today in your society?

$$
\text { Not A Little Very Extremely }
$$

Unsatisfied Unsatisfied Unsatisfied Unsatisfied Unsatisfied
A
$\mathrm{B}$
C
D
E

119. To what extent is your religion (or religion as a social institution) undergoing change in your society?

$\begin{array}{ccccc}\text { Not } & \text { Undergoing } & \text { Undergoing } & \text { Undergoing } & \text { Undergoing } \\ \text { Undergoing } & \text { A Little } & \text { Moderate } & \text { A Lot of } & \text { Extreme } \\ \text { Change } & \text { Change } & \text { Change } & \text { Change } & \text { Change } \\ \text { A } & B & C & \end{array}$

120. How much of a positive effect does this change have on the quality of your life?

Not

Positive

A

A Little

Positive

B

Positive

Very

Extremely

Positive

Positive

C

D

E 
121. How much of a negative effect does this change have on the quality or your life?

$\begin{array}{ccccc}\text { Not } & \text { A Little } & & \text { Very } & \text { Extremely } \\ \text { Negative } & \text { Negative } & \text { Negative } & \text { Negative } & \text { Negative } \\ \text { A } & \text { B } & \text { C } & \text { D } & \text { E }\end{array}$

122. To what extent are you participating in this change?
No
slight
Some
A Lot of
Maximum

Participation Participation Participation Participation Participation A

B

C

D

123. To what extent are other people participating in this change?
No
Slight
Some
A Lot of
Maximum

Participation Participation Participation Participation Participation A

B

C

D

124. To what extent is your religion (or religion as a social institution) open to influences in your society?

$\begin{array}{lcccc}\text { Not } & \text { A Little } & & \text { Very } & \text { Extremely } \\ \text { Open } & \text { Open } & \text { Open } & \text { Open } & \text { Open } \\ \text { A } & \text { B } & \text { C } & \text { D } & \text { E }\end{array}$

125. How much of a positive effect does this openness (or lack of openness) have on the quality of your life?

$\begin{array}{ccccc}\text { Not } & \text { A Little } & & \text { Very } & \text { Extremely } \\ \text { Positive } & \text { Positive } & \text { Positive } & \text { Positive } & \text { Positive } \\ \text { A } & \text { B } & \text { C } & \text { D } & \text { E }\end{array}$

126. How much of a negative effect does this openness (or lack of openness) have on the quality or your life? Not A Little Negative

A
Negative

B
Negative

C
Very Negative D
Extremely Negative

E

127. To what extent is your religion (or religion as a social institution) accessible to you in your society? (how feasible is it for you as a citizen to have access to, to make use of, or to contact this institution)

Not Accessible A
A Little Accessible
B
Very

Accessible

$\mathrm{c}$
Accessible Accessible 
128. How much of a positive effect does this accessibility (or lack of accessibility) have on the quality of your life?

$\begin{array}{ccccc}\text { Not } & \text { A Little } & & \text { Very } & \text { Extremely } \\ \text { Positive } & \text { Positive } & \text { Positive } & \text { Positive } & \text { Positive } \\ \text { A } & \text { B } & \text { C } & \text { D } & \text { E }\end{array}$

129. How much of a negative effect does this accessibility (or lack of accessibility) have on the quality or your life?

Not

Negative

A
A Little

Negative

B
Negative

c
Very

Negative

D
Extremely

Negative

E

130. To what extent is your religion (or religion as a social institution) supportive to you in your society? (how much does it serve your interests and needs or help to facilitate your goals)

$\begin{array}{ccccc}\text { Not } & \text { A Little } & & \text { Very } & \text { Extremely } \\ \text { Supportive } & \text { Supportive } & \text { Supportive } & \text { Supportive } & \text { Supportive } \\ \text { A } & \text { B } & \text { C } & \text { D } & \text { E }\end{array}$

131. How much of a positive effect does this support (or lack of support) have on the quality of your life?

$\begin{array}{ccccc}\text { Not } & \text { A Little } & & \text { Very } & \text { Extremely } \\ \text { Positive } & \text { Positive } & \text { Positive } & \text { Positive } & \text { Positive } \\ \text { A } & \text { B } & \text { C } & \text { D } & \text { E }\end{array}$

132. How much of a negative effect does this support (or lack of support) have on the quality or your life? Not A Little Negative Negative A B

Negative C

Very
Negative
D

D
Extremely Negative

$\mathrm{E}$

132a. We would like you to describe what, if anything, you consider good or positive about your religion (or religion as a social institution) in your society in terms of your thoughts, feelings, practices, experiences, or any other issues that are important to you. Please, describe in your own words on the DESCRIPTION SHEET provided: 
133. How positive is this issue in your life? (Please, circle one number: )

\begin{abstract}
Slightly
Positive
\end{abstract}

A
A Little

Positive

B
Very

Positive

C
Extremely Positive

$\mathrm{E}$

133a. We would like you to describe what, if anything, you consider distressful or troublesome about your religion (or religion as a social institution) in your society in terms of your thoughts, feelings. practices, experiences, or any other issues that are important to you. Please, describe in your own words on the DESCRIPTION SHEET provided:

134. How distressful is this issue in your life?

Slightly Distressful A
A Little Distressful

B
Distressful

C
Very Distressful D
Extremely Distressful 
(Not your family per se. but the family in your society in general)

135. Are you satisfied with the family (as a social institution) as it is today in your society?
(A) Yes
(B) No
(C) No Opinion
(D) Uncertain

136. How satisfied are you with the family in general (as a social institution) as it is today in your society?

$\begin{array}{ccccc}\text { Not } & \text { A Little } & & \text { Very } & \text { Extremely } \\ \text { Satisfied } & \text { Satisfied } & \text { Satisfied } & \text { Satisfied } & \text { Satisfied } \\ \text { A } & \text { B } & \text { C } & \text { D } & \text { E }\end{array}$

137. How unsatisfied are you with the family (as a social institution) as it is today in your society?

Not A Little Very Extremely Unsatisfied Unsatisfied Unsatisfied Unsatisfied Unsatisfied $\begin{array}{lllll}A & B & C & D & E\end{array}$

138. To what extent is the family (as a social institution) undergoing change in your society?

$\begin{array}{ccccc}\text { Not } & \text { Undergoing } & \text { Undergoing } & \text { Undergoing } & \text { Undergoing } \\ \text { Undergoing } & \text { A Little } & \text { Moderate } & \text { A Lot of } & \text { Extreme } \\ \text { Change } & \text { Change } & \text { Change } & \text { Change } & \text { Change } \\ \text { A } & \text { B } & \text { C } & \text { D } & \text { E }\end{array}$

139. How much of a positive effect does this change have on the quality of your life?

$\begin{array}{ccccc}\text { Not } & \text { A Little } & & \text { Very } & \text { Extremely } \\ \text { Positive } & \text { Positive } & \text { Positive } & \text { Positive } & \text { Positive } \\ \text { A } & \text { B } & \text { C } & \text { D } & \text { E }\end{array}$

140. How much of a negative effect does this change have on the quality or your 1 ife?

$\begin{array}{ccccc}\text { Not } & \text { A Little } & & \text { Very } & \text { Extremely } \\ \text { Negative } & \text { Negative } & \text { Negative } & \text { Negative } & \text { Negative } \\ \text { A } & \text { B } & \text { C } & \text { D } & \text { E }\end{array}$


141. To what extent are you participating in this change?
No
Slight
Some
A Lot of
Maximum

Participation Participation Participation Participation Participation $\begin{array}{lllll}A & B & C & D & E\end{array}$ E

142. To what extent are other people participating in this change?
No
Slight
Some
A Lot of
Maximum

Participation Participation Participation Participation Participation $\begin{array}{lllll}A & B & C & D & E\end{array}$

143. To what extent is the family (as a social institution) in your society open to influences?

$\begin{array}{lcccc}\text { Not } & \text { A Little } & & \text { Very } & \text { Extremely } \\ \text { Open } & \text { Open } & \text { Open } & \text { Open } & \text { Open } \\ \text { A } & \text { B } & \text { C } & \text { D } & \text { E }\end{array}$

144. How much of a positive effect does this openness (or lack of openness) have on the quality of your 1 ife?

$\begin{array}{ccccc}\text { Not } & \text { A Little } & & \text { Very } & \text { Extremely } \\ \text { Positive } & \text { Positive } & \text { Positive } & \text { Positive } & \text { Positive } \\ \text { A } & \text { B } & \text { C } & \text { D } & \text { E }\end{array}$

145. How much of a negative effect does this openness (or lack of openness) have on the quality or your life?

Not A Little

Negative

A
Negative

B
Negative

C
Very

Negative

D
Extremely

Negative

146. To what extent is the family (as a social institution) in your society supportive to you? (how much does it serve your interests and needs or help to facilitate your goals)
Not
A Little
Very
Extremely

Supportive Supportive

A

B

Supportive

C

Supportive

Supportive

D

E

147. How much of a positive effect does this support (or lack of support) have on the quality of your life?

Not

Positive

A
A Little

Positive

B
Positive

C
Very

Positive

D
Extremely

Positive

E 
148. How much of a negative effect does this support (or lack of support) have on the quality or your life?

$\begin{array}{ccccc}\text { Not } & \text { A Little } & & \text { Very } & \text { Extremely } \\ \text { Negative } & \text { Negative } & \text { Negative } & \text { Negative } & \text { Negative } \\ \text { A } & \text { B } & \text { C } & \text { D } & \text { E }\end{array}$

148a. We would 1ike you to describe what, if anything, you consider good or positive about the family (as a social institution) in your society in terms of your thoughts, feelings, practices, experiences, or any other issues that are important to you. Please, describe in your own words on the DESCRIPTION SHEET provided:

149. How positive is this issue in your life? (Please, circle one number: )

$\begin{array}{ccccc}\text { STightiy } & \text { A Little } & & \text { Very } & \text { Extremely } \\ \text { Positive } & \text { Positive } & \text { Positive } & \text { Positive } & \text { Positive } \\ \text { A } & \text { B } & \text { C } & \text { D } & \text { E }\end{array}$

149a. We would Tike you to describe what, if anything, you consider distressful or troublesome about the family (as a social institution) in your society in terms of your thoughts, feelings, practices,

experiences, or any other issues that are important to you. Please, describe in your own words on the DESCRIPTION SHEET provided:

150. How distressful is this issue in your Tife?

Slightly A Little Very Extremely Distressful Distressful Distressful Distressful Distressful

A

B
C 


\section{GENDER ROLES}

151. Are you satisfied with gender roles (as a social institution) as it is today in your society?
(A) Yes
(B) No
(C) No opinion
(D) Uncertain

152. How satisfied are you with gender roles (as a social institution) in your society? (please, circle one number:)

$\begin{array}{ccccc}\text { Not } & \text { A Little } & & \text { Very } & \text { Extremely } \\ \text { Satisfied } & \text { Satisfied } & \text { Satisfied } & \text { Satisfied } & \text { Satisfied } \\ \text { A } & \text { B } & \text { C } & \text { D } & \text { E }\end{array}$

153. How unsatisfied are you with gender roles (as a social institution) in your society?

Not A Little Very Extremely Unsatisfied Unsatisfied Unsatisfied Unsatisfied Unsatisfied $\begin{array}{lllll}A & B & C & D & E\end{array}$

154. To what extent are gender roles (as a social institution) undergoing change in your society?

$\begin{array}{ccccc}\text { Not } & \text { Undergoing } & \text { Undergoing } & \text { Undergoing } & \text { Undergoing } \\ \text { Undergoing } & \text { A Little } & \text { Moderate } & \text { A Lot of } & \text { Extreme } \\ \text { Change } & \text { Change } & \text { Change } & \text { Change } & \text { Change } \\ \text { A } & \text { B } & \text { C } & \text { D } & E\end{array}$

155. How much of a positive effect does this change have on the quality of your life?

$\begin{array}{ccccc}\text { Not } & \text { A Little } & & \text { Very } & \text { Extremely } \\ \text { Positive } & \text { Positive } & \text { Positive } & \text { Positive } & \text { Positive } \\ \text { A } & \text { B } & \text { C } & \text { D } & \text { E }\end{array}$

156. How much of a negative effect does this change have on the quality or your life?

$\begin{array}{ccccc}\text { Not } & \text { A Little } & & \text { Very } & \text { Extremely } \\ \text { Negative } & \text { Negative } & \text { Negative } & \text { Negative } & \text { Negative } \\ \text { A } & \text { B } & \text { C } & \text { D } & \text { E }\end{array}$

157. To what extent are you participating in this change?

No Slight Some A Lot of Maximum

Participation Participation Participation Participation Participation A 
158. To what extent are other people participating in this change?
No
Slight
Some
A Lot of
Maximum

Participation Participation Participation Participation Participation A
B
C
D

159. To what extent are gender roles (as a social institution) open to influences in your society?

$\begin{array}{lcccc}\text { Not } & \text { A Little } & & \text { Very } & \text { Extremely } \\ \text { Open } & \text { Open } & \text { Open } & \text { Open } & \text { Open } \\ \text { A } & \text { B } & \text { C } & \text { D } & \text { E }\end{array}$

160. How much of a positive effect does this openness (or lack of openness) have on the quality of your 1 ife?

Not A Little Very Extremely

Positive Positive Positive Positive Positive

A

B

C

D

E

161. How much of a negative effect does this openness (or lack of openness) have on the quality or your life?

Not

Negative

A
A Little

Negative

B
Very

Negative Negative

Negative

C

D

E

162. To what extent are gender roles (as a social institution)

supportive to you in your society? (how much do they serve your interests and needs or help to facilitate your goals)

$\begin{array}{ccccc}\text { Not } & \text { A Little } & & \text { Very } & \text { Extremely } \\ \text { Supportive } & \text { Supportive } & \text { Supportive } & \text { Supportive } & \text { Supportive } \\ \text { A } & \text { B } & \text { C } & \text { D } & \text { E }\end{array}$

163. How much of a positive effect does this support (or lack of support) have on the quality of your life?

$\begin{array}{ccccc}\text { Not } & \text { A Little } & & \text { Very } & \text { Extremely } \\ \text { Positive } & \text { Positive } & \text { Positive } & \text { Positive } & \text { Positive } \\ \text { A } & \text { B } & \text { C } & \text { D } & \text { E }\end{array}$

164. How much of a negative effect does this support (or lack of support) have on the quality or your life?

Not A Little

Negative

A
Negative

B
Negative

c
Very

Negative

D
Extremely Negative 
164a. We would like you to describe what, if anything, you consider good or positive about gender roles in your society in terms of your thoughts, feelings, practices, experiences, or any other issues that are important to you. Please, describe in your own words on the DESCRIPTION SHEET provided:

165. How positive is this issue in your life?

$\begin{array}{ccccc}\text { Slightly } & \text { A Little } & & \text { Very } & \text { Extremely } \\ \text { Positive } & \text { Positive } & \text { Positive } & \text { Positive } & \text { Positive } \\ \text { A } & \text { B } & \text { C } & \text { D } & \text { E }\end{array}$

165a. We would like you to describe what, if anything, you consider distressful or troublesome about gender roles in your society in terms of your thoughts, feelings, practices, experiences, or any other issues that are important to you. Please, describe in your own words on the DESCRIPTION SHEET provided:

166. How distressful is this issue in your life?

Slightly A Little Very Extremely Distressful Distressful Distressful Distressful Distressful

A

B

C

D 
APPENDIX C

183 
Table C1

Factor Structure Matrix of the Identity Domains Scale in Miami

Rotated factor matrix

Content areas

\begin{tabular}{lll}
\hline Factor 1 & Factor 2 & Factor 3
\end{tabular}

Career goals

.795

Sense of self

.625

.319

Long-terms goals

.810

Friendship

.510

Belonging to Groups

.731

Sexuality issues

.723

Religious issues

.636

Morality issues

.705

Political issues

.791

Eigenvalue $=$

3.13

1.07

.94

Variance $=$

$34.8 \%$

$11.8 \%$

$10.5 \%$

Total variance $=\quad 57.2 \%$ 
Table C2

Factor Structure Matrix of the Identity Domains Scale in Nebraska

Rotated factor matrix

Content areas

\begin{tabular}{lll}
\hline Factor 1 & Factor 2 & Factor 3
\end{tabular}

Career goals

.784

.340

Sense of self

.680

.334

Long-terms goals

.756

Friendship

.757

Belonging to groups

.581

Sexuality issues

.577

Religious issues

.568

Morality issues

.521

.518

Political issues

.894

Eigenvalue $=$

3.13

1.07

.99

Variance $=$

$34.9 \%$

$11.9 \%$

$11.0 \%$

Total variance $=\quad 57.7 \%$ 
Table C3

Factor Structure Matrix of the Identity Domains Scale in Brazil

Rotated factor matrix

Content areas

\begin{tabular}{lll}
\hline Factor 1 & Factor 2 & Factor 3
\end{tabular}

Career goals

.785

Sense of self

.631

.437

Long-terms goals

.356

.731

Friendship

.837

Belonging to groups

.841

Sexuality issues

.638

.326

Religious issues

.747

Morality issues

.718

Political issues

.547

Eigenvalue=

3.04

1.29

1.06

Variance $=$

$33.7 \%$

$14.4 \%$

$11.8 \%$

Total variance $=\quad 59.9 \%$ 
Table C4

Factor Structure Matrix of the Identity Domains Scale in Costa Rica

Rotated factor matrix

Content areas

\begin{tabular}{lll}
\hline Factor 1 & Factor 2 & Factor 3
\end{tabular}

Career goals

.360

.324

.527

Sense of self

.344

.515

.423

Long-terms goals

.811

Friendship

.737

Belonging to groups

.799

Sexuality issues

.645

Religious issues

.715

Morality issues

.774

.304

Political issues

.436

.502

$-.450$

Eigenvalue=

2.89

1.28

1.21

Variance $=$

$32.1 \%$

$14.2 \%$

$13.4 \%$

Total variance $=\quad 59.8 \%$ 
Table C5

Factor Structure Matrix of The Identity Domains Scale in China

Rotated factor matrix

Content areas

\begin{tabular}{lll}
\hline Factor 1 & Factor 2 & Factor 3
\end{tabular}

Career goals

.566

Sense of self

.763

.335

Long-terms goals

.386

.683

Friendship

.440

.689

Belonging to groups

Sexuality issues

.707

Religious issues

.522

.413

Morality issues

.794

Political issues

.472

Eigenvalue $=$

2.57

1.32

1.04

Variance $=$

$28.5 \%$

$14.7 \%$

$11.7 \%$

Total variance $=\quad 54.9 \%$ 
Table C6

Factor Structure Matrix of the Identity Domains Scale in Sweden

Rotated factor matrix

Content areas

\begin{tabular}{lll}
\hline Factor 1 & Factor 2 & Factor 3
\end{tabular}

Career goals

.713

Sense of self

.325

.818

Long-terms goals

.833

Friendship

.754

Belonging to groups

.637

Sexuality issues

.550

$-.558$

Religious issues

.504

Morality issues

.672

Political issues

.322

$\begin{array}{llll}\text { Eigenvalue }= & 2.05 & 1.51 & 1.21 \\ \text { Variance }= & 22.7 \% & 16.9 \% & 13.5 \% \\ \text { Total variance= } & 53.1 \% & & \end{array}$


Table 1

Gender Distribution, Expected Value, and Adjusted Standardized Residuals by Site

\begin{tabular}{|c|c|c|c|c|}
\hline Site & & Male & Female & $\begin{array}{l}\text { Row } \\
\text { total }\end{array}$ \\
\hline Miami & $\begin{array}{l}\text { Count } \\
\text { Exp val } \\
\text { Row pct } \\
\text { Col pct } \\
\text { Adj Res }\end{array}$ & $\begin{array}{l}54 \\
95.3 \\
28.6 \% \\
12.2 \% \\
-6.8\end{array}$ & $\begin{array}{l}135 \\
93.7 \\
71.4 \% \\
31.1 \% \\
6.8\end{array}$ & $\begin{array}{l}189 \\
21.6 \%\end{array}$ \\
\hline Nebraska & $\begin{array}{l}\text { Count } \\
\text { Exp Val } \\
\text { Row Pct } \\
\text { Col Pct } \\
\text { Adj Res }\end{array}$ & $\begin{array}{l}108 \\
96.8 \\
56.3 \% \\
24.5 \% \\
1.8\end{array}$ & $\begin{array}{l}84 \\
95.2 \\
43.8 \% \\
19.4 \% \\
-1.8\end{array}$ & $\begin{array}{l}192 \\
21.9 \%\end{array}$ \\
\hline Brazil & $\begin{array}{l}\text { Count } \\
\text { Exp Val } \\
\text { Row Pct } \\
\text { Col Pct } \\
\text { Adj Res }\end{array}$ & $\begin{array}{l}111 \\
82.7 \\
67.7 \% \\
25.2 \% \\
4.9\end{array}$ & $\begin{array}{l}53 \\
81.3 \\
32.3 \% \\
12.2 \% \\
-4.9\end{array}$ & $\begin{array}{l}\overline{164} \\
18.7 \%\end{array}$ \\
\hline Costa Rica & $\begin{array}{l}\text { Count } \\
\text { Exp Val } \\
\text { Row Pct } \\
\text { Col Pct } \\
\text { Adj Res }\end{array}$ & $\begin{array}{l}74 \\
63.5 \\
58.7 \% \\
25.2 \% \\
4.9\end{array}$ & $\begin{array}{l}52 \\
62.5 \\
41.3 \% \\
12.2 \% \\
-4.9\end{array}$ & $\begin{array}{l}126 \\
14.4 \%\end{array}$ \\
\hline China & $\begin{array}{l}\text { Count } \\
\text { Exp Val } \\
\text { Row Pct } \\
\text { Col Pct } \\
\text { Adj Res }\end{array}$ & $\begin{array}{l}60 \\
58.0 \\
52.2 \% \\
13.6 \% \\
.4\end{array}$ & $\begin{array}{l}55 \\
57.0 \\
47.8 \% \\
12.7 \% \\
-.4\end{array}$ & $\begin{array}{l}\overline{115} \\
13.1 \%\end{array}$ \\
\hline Sweden & $\begin{array}{l}\text { Count } \\
\text { Exp Val } \\
\text { Row Pct } \\
\text { Col Pct } \\
\text { Adj Res }\end{array}$ & $\begin{array}{l}34 \\
44.9 \\
38.2 \% \\
7.7 \% \\
-2.4\end{array}$ & $\begin{array}{l}55 \\
44.1 \\
61.8 \% \\
12.7 \% \\
2.4\end{array}$ & $\begin{array}{l}89 \\
10.2 \%\end{array}$ \\
\hline & $\begin{array}{l}\text { Column } \\
\text { Total }\end{array}$ & $\begin{array}{l}441 \\
50.4 \%\end{array}$ & $\begin{array}{l}434 \\
49.6 \%\end{array}$ & $\begin{array}{l}875 \\
100.0 \%\end{array}$ \\
\hline
\end{tabular}

Note. $X^{2}(875,5)=67.19, p<.00001$.

${ }^{a}$ Expected value.

${ }^{b}$ Row percentage.

c Column Percentage.

d Adjusted standardized residuals. 
Table 2

Grade Distribution, Expected Value, And Adjusted Standardized Residuals by Site

\begin{tabular}{|c|c|c|c|c|}
\hline & & First 2 yrs & $\begin{array}{l}\text { Other than } \\
\text { first } 2 \text { yrs }\end{array}$ & $\begin{array}{l}\text { Row } \\
\text { total }\end{array}$ \\
\hline Miami & $\begin{array}{l}\text { Count } \\
\text { Exp Val } \\
\text { Row Pct }^{\mathrm{a}} \\
\text { Col Pct } \\
\text { Adj Res }\end{array}$ & $\begin{array}{l}137 \\
145.8 \\
74.1 \% \\
20.1 \% \\
-1.8\end{array}$ & $\begin{array}{l}48 \\
39.2 \\
25.9 \% \\
26.2 \% \\
1.8\end{array}$ & $\begin{array}{l}185 \\
21.4 \%\end{array}$ \\
\hline Nebraska & $\begin{array}{l}\text { Count } \\
\text { Exp Val } \\
\text { Row Pct } \\
\text { Col Pct } \\
\text { Adj Res }\end{array}$ & $\begin{array}{l}165 \\
149.8 \\
86.8 \% \\
24.2 \% \\
3.1\end{array}$ & $\begin{array}{l}25 \\
40.2 \\
13.2 \% \\
13.7 \% \\
-3.1\end{array}$ & $\begin{array}{l}190 \\
22.0 \%\end{array}$ \\
\hline Brazil & $\begin{array}{l}\text { Count } \\
\text { Exp Val } \\
\text { Row Pct } \\
\text { Col Pct } \\
\text { Adj Res }\end{array}$ & $\begin{array}{l}150 \\
128.5 \\
92.0 \% \\
22.0 \% \\
4.6\end{array}$ & $\begin{array}{l}13 \\
34.5 \\
8.0 \% \\
7.1 \% \\
-4.6\end{array}$ & $\begin{array}{l}163 \\
18.9 \%\end{array}$ \\
\hline Costa Rica & $\begin{array}{l}\text { Count } \\
\text { Exp Val } \\
\text { Row Pct } \\
\text { Col Pct } \\
\text { Adj Res }\end{array}$ & $\begin{array}{l}64 \\
97.7 \\
51.6 \% \\
9.4 \% \\
-8.0\end{array}$ & $\begin{array}{l}60 \\
26.3 \\
48.4 \% \\
32.8 \% \\
8.0\end{array}$ & $\begin{array}{l}124 \\
14.4 \%\end{array}$ \\
\hline China & $\begin{array}{l}\text { Count } \\
\text { Exp Val } \\
\text { Row Pct } \\
\text { Col Pct } \\
\text { Adj Res }\end{array}$ & $\begin{array}{l}111 \\
90.6 \\
96.5 \% \\
16.3 \% \\
5.0\end{array}$ & $\begin{array}{l}4 \\
24.4 \\
3.5 \% \\
2.2 \% \\
-5.0\end{array}$ & $\begin{array}{l}\overline{115} \\
13.3 \%\end{array}$ \\
\hline Sweden & $\begin{array}{l}\text { Count } \\
\text { Exp Val } \\
\text { Row Pct } \\
\text { Col Pct } \\
\text { Adj Res }\end{array}$ & $\begin{array}{l}54 \\
68.6 \\
62.1 \% \\
7.9 \% \\
-4.0\end{array}$ & $\begin{array}{l}33 \\
18.4 \\
37.9 \% \\
18.0 \% \\
4.0\end{array}$ & $\begin{array}{l}87 \\
10.1 \%\end{array}$ \\
\hline & $\begin{array}{l}\text { Column } \\
\text { Total }\end{array}$ & $\begin{array}{l}681 \\
78.8 \%\end{array}$ & $\begin{array}{l}183 \\
21.2 \%\end{array}$ & $\begin{array}{l}\overline{864} \\
100.0 \%\end{array}$ \\
\hline
\end{tabular}

Note. $X^{2}(5, N=864)=118.05, p<.00001$.

${ }^{a}$ Expected value.

${ }^{b}$ Row percentage.

${ }^{c}$ Column Percentage.

${ }^{d}$ Adjusted standardized residuals. 
Table 3

Marital Status Distribution, Expected Value, and Adjusted Standardized Residuals by Site

\begin{tabular}{|c|c|c|c|c|}
\hline & & Single & $\begin{array}{l}\text { Other } \\
\text { than Single }\end{array}$ & $\begin{array}{l}\text { Row } \\
\text { Total }\end{array}$ \\
\hline Miami & $\begin{array}{l}\text { Count } \\
\text { Exp Val } \\
\text { Row Pct }^{a} \\
\text { Col Pct } \\
\text { Adj Res }\end{array}$ & $\begin{array}{l}178 \\
167.0 \\
92.7 \% \\
23.4 \% \\
2.7\end{array}$ & $\begin{array}{l}14 \\
25.0 \\
7.3 \% \\
12.3 \% \\
-2.7\end{array}$ & $\begin{array}{l}192 \\
22.0 \%\end{array}$ \\
\hline Nebraska & $\begin{array}{l}\text { Count } \\
\text { Exp Val } \\
\text { Row Pct } \\
\text { Col Pct } \\
\text { Adj Res }\end{array}$ & $\begin{array}{l}191 \\
167.8 \\
99.0 \% \\
25.1 \% \\
5.6\end{array}$ & $\begin{array}{l}2 \\
25.2 \\
1.0 \% \\
1.8 \% \\
-5.6\end{array}$ & $\begin{array}{l}193 \\
22.1 \%\end{array}$ \\
\hline Brazil & $\begin{array}{l}\text { Count } \\
\text { Exp Val } \\
\text { Row Pct } \\
\text { Col Pct } \\
\text { Adj Res }\end{array}$ & $\begin{array}{l}148 \\
140.0 \\
91.9 \% \\
19.5 \% \\
2.1\end{array}$ & $\begin{array}{l}13 \\
21.0 \\
8.1 \% \\
11.4 \% \\
-2.1\end{array}$ & $\begin{array}{l}161 \\
18.4 \%\end{array}$ \\
\hline Costa Rica & $\begin{array}{l}\text { Count } \\
\text { Exp Val } \\
\text { Row Pct } \\
\text { Col Pct } \\
\text { Adj Res }\end{array}$ & $\begin{array}{l}56 \\
109.6 \\
44.4 \% \\
7.4 \% \\
-15.3\end{array}$ & $\begin{array}{l}70 \\
16.4 \\
55.6 \% \\
61.4 \% \\
15.3\end{array}$ & $\begin{array}{l}126 \\
13.3 \%\end{array}$ \\
\hline China & $\begin{array}{l}\text { Count } \\
\text { Exp Val } \\
\text { Row Pct } \\
\text { Col Pct } \\
\text { Adj Res }\end{array}$ & $\begin{array}{l}116 \\
100.9 \\
100.0 \% \\
15.3 \% \\
4.5\end{array}$ & $\begin{array}{l}0 \\
15.1 \\
.0 \% \\
.0 \% \\
-4.5\end{array}$ & $\begin{array}{l}116 \\
13.3 \%\end{array}$ \\
\hline Sweden & $\begin{array}{l}\text { Count } \\
\text { Exp Val } \\
\text { Row Pct } \\
\text { Col Pct } \\
\text { Adj Res }\end{array}$ & $\begin{array}{l}71 \\
74.8 \\
82.6 \% \\
9.3 \% \\
-1.3\end{array}$ & $\begin{array}{l}15 \\
11.2 \\
17.4 \% \\
13.2 \% \\
1.3\end{array}$ & $\begin{array}{l}86 \\
9.8 \%\end{array}$ \\
\hline & $\begin{array}{l}\text { Column } \\
\text { Total }\end{array}$ & $\begin{array}{l}760 \\
87.0 \%\end{array}$ & $\begin{array}{l}114 \\
13.0 \%\end{array}$ & $\begin{array}{l}874 \\
100.0 \%\end{array}$ \\
\hline
\end{tabular}

Note: $X^{2}(5, N=874)=253.28$, p. $<.00001$.

${ }^{a}$ Expected value.

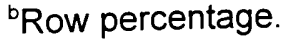

${ }^{\circ}$ Column Percentage.

${ }^{\mathrm{d} A d j u s t e d}$ standardized residuals. 
Reliability Coefficients for the Total Identity Satisfaction, Personal, Interpersonal. and World View Satisfaction Scales by Site.

\begin{tabular}{llllll} 
& \multicolumn{5}{c}{ Alpha coefficient $(\propto)$} \\
\cline { 2 - 6 } & $\begin{array}{l}\text { Total } \\
\text { identity } \\
\text { satisfaction }\end{array}$ & $\begin{array}{l}\text { Personal } \\
\text { identity } \\
\text { satisfaction }\end{array}$ & $\begin{array}{l}\text { Interpersonal } \\
\text { identity } \\
\text { satisfaction }\end{array}$ & $\begin{array}{l}\text { World } \\
\text { view } \\
\text { satisfaction }\end{array}$ & $\begin{array}{l}\text { N of } \\
\text { cases }\end{array}$ \\
\cline { 2 - 6 } Nebraska & .76 & .68 & .47 & .50 & 172 \\
Brazil & .74 & .69 & .53 & .41 & 180 \\
Costa Rica & .73 & .72 & .60 & .37 & 163 \\
China & .68 & .62 & .54 & .49 & 114 \\
Sweden & .55 & .42 & .38 & .48 & 97 \\
N of Items & 9 & 3 & .52 & .03 & 84 \\
\end{tabular}


Table 5

Factor Structure Matrix of the Attribution and Evaluation Rating Across Social $\underline{\text { Institutions in Miami }}$

Rotated component matrix

Institutional ratings

Factor $1 \quad$ Factor 2

Access

.578

.425

Change

.828

Openness

.694

Participation

.805

Support

.577

.602

Pos or neg access

.771

Pos or neg change

.762

Pos or neg openness

.814

Satisfaction/Dissatisfaction

.651

Pos or neg support

.836
Variance $=$
$36.9 \%$
$24.6 \%$
Eigenvalue $=3.69$
2.46
Total $\%$ of variance $=61.4$

Note. aPositive or negative effect. 
Table 6

Factor Structure Matrix of the Attribution and Evaluation Rating Across Social Institutions in Nebraska

Institutional ratings

Rotated component matrix

Factor $1 \quad$ Factor 2

Access

.491

.565

Change

.861

Openness

.302

.703

Participation

.787

Support

.674

.509

Pos or neg access

.778

Pos or neg change

.835

Pos or neg openness

.814

Satisfaction/Dissatisfaction

.823

Pos or neg support

.871

\begin{tabular}{ll} 
Variance $=\quad 42.0 \%$ & $25.7 \%$ \\
Eigenvalue $=4.20$ & 2.57 \\
Total $\%$ of variance $=67.7$ & \\
\hline
\end{tabular}

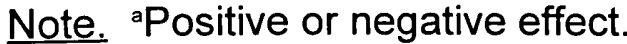


Table 7

Factor Structure Matrix of the Attribution and Evaluation Rating Across Social

Institutions in Brazil

Institutional ratings

Rotated component matrix

Factor $1 \quad$ Factor 2

Access

.384

.699

Change

.789

Openness

.394

.675

Participation

.791

Support

.571

.442

Pos or nega access

.791

.306

Pos or neg change

.697

Pos or neg openness

.804

Satisfaction/Dissatisfaction

.703

Pos or neg support

.881

$\begin{array}{ll}\text { Variance }=36.6 \% & 25.8 \% \\ \text { Eigenvalue }=3.66 & 2.58 \\ \text { Total } \% \text { of variance }=62.4 & \end{array}$

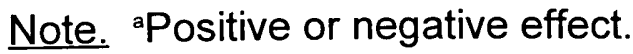


Table 8

Factor Structure Matrix of the Attribution and Evaluation Rating Across Social Institutions in Costa Rica

Institutional ratings

Rotated component matrix

Factor $1 \quad$ Factor 2

Access

.567

.544

Change

.858

Openness

.795

Participation

.713

Support

.711

.469

Pos or nega access

.788

Pos or neg change

.872

Pos or neg openness

.838

Satisfaction/Dissatisfaction

.815

Pos or neg support

.869

$\begin{array}{ll}\text { Variance }=\quad 43.6 \% & 24.6 \% \\ \text { Eigenvalue }=4.36 & 2.46 \\ \text { Total } \% \text { of variance }=68.2 & \end{array}$

Note. ${ }^{\text {a }}$ ositive or negative effect. 
Table 9

Factor Structure Matrix of the Attribution and Evaluation Rating Across Social Institutions in China

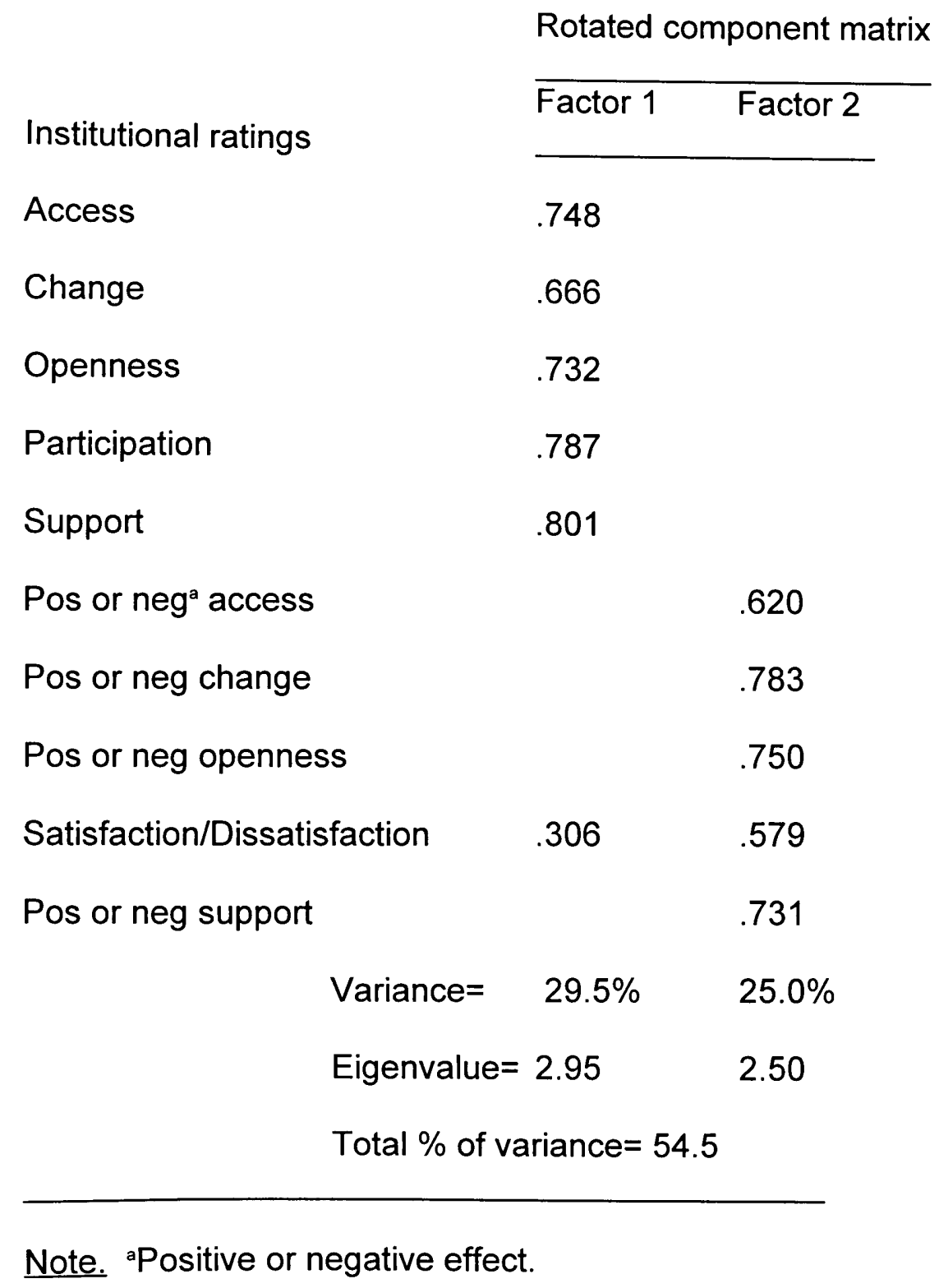


Table 10

Factor Structure Matrix of the Attribution and Evaluation Rating Across Social Institutions in Sweden

Institutional ratings

Access

.705

Change

Rotated component matrix

Factor $1 \quad$ Factor 2

Openness

.780

Participation

.498

.511

Support

.794

Pos or neg access

.796

Pos or neg change

.750

Pos or neg openness

.866

Satisfaction/Dissatisfaction

.688

$-.356$

Pos or neg support

.880

$\begin{array}{ll}\text { Variance }=\quad 47.4 \% & 16.8 \% \\ \text { Eigenvalue }=4.74 & 1.68 \\ \text { Total } \% \text { of Variance }=64.2 & \end{array}$

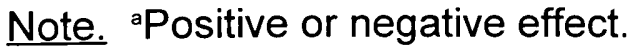




\section{Table 11}

Reliability Coefficients for the Overall Institutional Attributes Scale and the

Overall Institutional Evaluation Scale

\begin{tabular}{lll} 
Site & $\begin{array}{l}\text { Institutional } \\
\text { attribute } \\
\text { scale } \\
\text { alpha( } \propto \text { ) }\end{array}$ & $\begin{array}{l}\text { Institutional } \\
\text { evaluation } \\
\text { scale } \\
\text { alpha }(\propto)\end{array}$ \\
\hline Miami & .73 & .79 \\
n of cases & 188 & 187 \\
\hline Nebraska & .80 & .84 \\
$n$ of cases & 192 & 192 \\
\hline Brazil & .73 & .82 \\
$n$ of cases & 154 & 154 \\
\hline Costa Rica & .74 & .87 \\
$n$ of cases & 121 & 116 \\
\hline China & .75 & .62 \\
n of cases & 115 & 115 \\
\hline Sweden & .63 & .84 \\
n of cases & 89 & 87 \\
\hline N of items & 5 & 5 \\
\hline
\end{tabular}


Table 12

Reliability Coefficients for the Institutional Attributes Scale of Social Institutions by Site

Site $\quad$ Gov $^{\mathrm{a}}$ Leg $^{\mathrm{b}} \mathrm{Pol}^{\mathrm{c}} \mathrm{Rel}^{\mathrm{d}} \mathrm{Fam}^{\mathrm{e}} \mathrm{Gdr}^{\mathrm{f}}$

\begin{tabular}{llllllll}
\hline Miami & alpha & .51 & .57 & .63 & .66 & .45 & .58 \\
& $n$ of cases & 192 & 191 & 191 & 187 & 187 & 186 \\
& $n$ of items & 6 & 6 & 6 & 6 & 5 & 5
\end{tabular}

\begin{tabular}{llllllll}
\hline Nebraska & alpha & .56 & .65 & .69 & .69 & .57 & .63 \\
& $n$ of cases & 193 & 192 & 191 & 190 & 192 & 190 \\
& $n$ of items & 6 & 6 & 6 & 6 & 5 & 5
\end{tabular}

\begin{tabular}{llllllll}
\hline Brazil & alpha & .54 & .73 & .67 & .73 & .69 & .76 \\
& $n$ of cases & 164 & 161 & 159 & 152 & 152 & 153 \\
& $n$ of items & 6 & 6 & 6 & 6 & 5 & 5
\end{tabular}

\begin{tabular}{llllllll}
\hline Costa Rica & alpha & .44 & .67 & .68 & .73 & .63 & .53 \\
& n of cases & 128 & 125 & 63 & 126 & 128 & 129 \\
& n of items & 6 & 6 & 6 & 6 & 5 & 5
\end{tabular}

\begin{tabular}{llllllll}
\hline China & alpha & N/A & .63 & N/A & .63 & .54 & .66 \\
& $n$ of cases & N/A & 113 & N/A & 116 & 115 & 116 \\
& $N$ of items & N/A & 6 & N/A & 6 & 5 & 5
\end{tabular}

\begin{tabular}{llllllll}
\hline Sweden & alpha & .49 & .66 & .58 & .64 & .57 & .46 \\
& $\mathrm{n}$ of cases & 89 & 90 & 90 & 89 & 89 & 89 \\
& $\mathrm{n}$ of items & 6 & 6 & 6 & 6 & 5 & 5
\end{tabular}

Note. ${ }^{a}$ Government.

Legal/Judicial institutions.

'Political Institutions.

${ }^{\mathrm{C}}$ Religious institutions.

${ }^{d}$ Family.

${ }^{\mathrm{e}}$ Gender roles. 
Table 13

Reliability Coefficients for the Evaluation Scale of Social Institutions by Site

Site

Fam $^{a}$ Gdr ${ }^{b}$ Gov $^{c}$ Leg $^{d}$ Pol $^{e} \quad$ Rel $^{f}$

\begin{tabular}{llllllll}
\hline Miami & alpha & .74 & .72 & .68 & .79 & .68 & .81 \\
& n of cases & 180 & 173 & 188 & 187 & 183 & 185 \\
& $n$ of items & 5 & 5 & 5 & 5 & 5 & 5
\end{tabular}

\begin{tabular}{llllllll}
\hline Nebraska & alpha & .79 & .60 & .75 & .81 & .81 & .85 \\
& $n$ of cases & 189 & 184 & 189 & 188 & 187 & 188 \\
& $n$ of items & 5 & 5 & 5 & 5 & 5 & 5
\end{tabular}

\begin{tabular}{llllllll}
\hline Brazil & alpha & .77 & .67 & .78 & .78 & .77 & .82 \\
& $n$ of cases & 147 & 140 & 158 & 151 & 154 & 149 \\
& $n$ of items & 5 & 5 & 5 & 5 & 5 & 5
\end{tabular}

\begin{tabular}{llllllll}
\hline Costa Rica & alpha & .71 & .79 & .81 & .80 & .84 & .76 \\
& $n$ of cases & 126 & 126 & 124 & 125 & 62 & 125 \\
& $n$ of items & 4 & 4 & 4 & 4 & 4 & 4
\end{tabular}

\begin{tabular}{llllllll}
\hline China & alpha & .59 & .47 & N/A & .62 & N/A & .51 \\
& $\begin{array}{llllll}\text { n of cases } \\
\text { N of items }\end{array}$ & 4 & 4 & 4 & 4 & 4 & 4 \\
\hline Sweden & alpha & .81 & .72 & .66 & .82 & .81 & .81 \\
& $\begin{array}{l}n \text { of cases } \\
\text { n of items }\end{array}$ & 5 & 73 & 74 & 75 & 75 & 72 \\
& & 5 & 5 & 5 & 5
\end{tabular}

Note. ${ }^{a}$ Family.

${ }^{b}$ Gender roles.

${ }^{\mathrm{c} G o v e r n m e n t . ~}$

'Legal/Judicial institutions.

dPolitical Institutions.

e'Religious institutions. 
Table 14

Personal Development Status Distribution, Expected Value, and Adjusted Standardized Residuals by Site

\begin{tabular}{|c|c|c|c|c|c|c|}
\hline Site & & Diffa $^{a}$ & Fore $^{b}$ & Mora $^{c}$ & $A_{c h i}{ }^{d}$ & Tota \\
\hline Miami & $\begin{array}{l}\text { Count } \\
\text { Exp Val } \\
\text { Row Pct } \\
\text { Col Pct } \\
\text { Adj Res }^{\mathrm{h}}\end{array}$ & $\begin{array}{l}11 \\
21.7 \\
7.0 \% \\
11.6 \% \\
-2.8\end{array}$ & $\begin{array}{l}31 \\
30.0 \\
19.6 \% \\
23.7 \% \\
.2\end{array}$ & $\begin{array}{l}35 \\
32.7 \\
22.2 \% \\
24.5 \% \\
.5\end{array}$ & $\begin{array}{l}81 \\
73.6 \\
51.3 \% \\
25.2 \% \\
1.3\end{array}$ & 158 \\
\hline Nebraska & $\begin{array}{l}\text { Count } \\
\text { Exp Val } \\
\text { Row Pct } \\
\text { Col Pct } \\
\text { Adj Res }\end{array}$ & $\begin{array}{l}16 \\
22.7 \\
9.7 \% \\
16.8 \% \\
-1.7\end{array}$ & $\begin{array}{l}22 \\
31.3 \\
13.3 \% \\
16.8 \% \\
-2.1\end{array}$ & $\begin{array}{l}62 \\
34.1 \\
37.6 \% \\
43.4 \% \\
6.1\end{array}$ & $\begin{array}{l}65 \\
76.9 \\
39.4 \% \\
20.2 \% \\
-2.1\end{array}$ & 165 \\
\hline
\end{tabular}

\begin{tabular}{|c|c|c|c|c|c|c|}
\hline Brazil & $\begin{array}{l}\text { Count } \\
\text { Exp Val } \\
\text { Row Pct } \\
\text { Col Pct } \\
\text { Adj Res }\end{array}$ & $\begin{array}{l}28 \\
20.5 \\
18.8 \% \\
29.5 \% \\
2.0\end{array}$ & $\begin{array}{l}23 \\
28.2 \\
15.4 \% \\
17.6 \% \\
-1.2\end{array}$ & $\begin{array}{l}22 \\
30.8 \\
14.8 \% \\
15.4 \% \\
-2.0\end{array}$ & $\begin{array}{l}76 \\
69.4 \\
51.0 \% \\
23.6 \% \\
1.2\end{array}$ & 149 \\
\hline Costa Rica & $\begin{array}{l}\text { Count } \\
\text { Exp Val } \\
\text { Row Pct } \\
\text { Col Pct } \\
\text { Adj Res }\end{array}$ & $\begin{array}{l}9 \\
12.1 \\
10.2 \% \\
9.5 \% \\
-1.0\end{array}$ & $\begin{array}{l}6 \\
16.7 \\
6.8 \% \\
4.6 \% \\
-3.1\end{array}$ & $\begin{array}{l}13 \\
18.2 \\
14.8 \% \\
9.1 \% \\
-1.5\end{array}$ & $\begin{array}{l}60 \\
41.0 \\
68.2 \% \\
18.6 \% \\
4.3\end{array}$ & 88 \\
\hline
\end{tabular}

\begin{tabular}{lllllll}
\hline China & Count & 17 & 17 & 10 & 27 & 71 \\
& Exp Val & 9.8 & 13.5 & 14.7 & 33.1 & \\
& Row Pct & $23.9 \%$ & $23.9 \%$ & $14.1 \%$ & $38.0 \%$ \\
& Col Pct & $17.9 \%$ & $13.0 \%$ & $7.0 \%$ & $8.4 \%$ & -1.5 \\
& Adj Res & 2.6 & 1.1 & -1.5 & & \\
& & & & & 13 & 60 \\
& Count & 14 & 32 & 1 & 28.0 & \\
& Exweden & Row Pct & $23.3 \%$ & 11.4 & 12.4 & $21.7 \%$ \\
& Col Pct & $14.7 \%$ & $24.4 \%$ & $1.7 \%$ & $4.0 \%$ & \\
& Adj Res & 2.3 & 7.1 & $-7 \%$ & -4.1
\end{tabular}

Total Count

691

\begin{tabular}{|c|c|}
\hline $\begin{array}{lll}{ }^{D} \text { Diffused } & { }^{b} \text { Foreclosed } & \text { cMoratorium } \\
\text { Expected value } & { }^{\text {ER }} \text { Row percentage } & { }^{n} \text { Column percentage }\end{array}$ & $\begin{array}{l}{ }^{\text {AAchieved. }} \\
\text { 'Adjusted standardized residuals }\end{array}$ \\
\hline
\end{tabular}


Table 15

Interpersonal Development Status Distribution, Expected Value, and Adjusted Standardized Residuals by Site

\begin{tabular}{|c|c|c|c|c|c|c|}
\hline Site & & Diff $^{a}$ & Fore $^{b}$ & Mora $^{c}$ & Achi $^{d}$ & Total \\
\hline Miami & $\begin{array}{l}\text { Count } \\
\text { Exp Val } \\
\text { Row Pct } \\
\text { Col Pct } \\
\text { Adj Res }\end{array}$ & $\begin{array}{l}6 \\
20.4 \\
4.2 \% \\
7.1 \% \\
-4.0\end{array}$ & $\begin{array}{l}52 \\
51.0 \\
36.4 \% \\
24.8 \% \\
.2\end{array}$ & $\begin{array}{l}10 \\
15.5 \\
7.0 \% \\
15.6 \% \\
-1.7\end{array}$ & $\begin{array}{l}75 \\
56.1 \\
52.4 \% \\
32.5 \% \\
3.7\end{array}$ & $\overline{143}$ \\
\hline Nebraska & $\begin{array}{l}\text { Count } \\
\text { Exp Val } \\
\text { Row Pct } \\
\text { Col Pct } \\
\text { Adj Res }\end{array}$ & $\begin{array}{l}5 \\
20.8 \\
3.4 \% \\
6.0 \% \\
-4.3\end{array}$ & $\begin{array}{l}66 \\
52.1 \\
45.2 \% \\
31.4 \% \\
2.8\end{array}$ & $\begin{array}{l}19 \\
15.9 \\
13.0 \% \\
29.7 \% \\
1.0\end{array}$ & $\begin{array}{l}56 \\
57.3 \\
38.4 \% \\
24.2 \% \\
-.2\end{array}$ & $\overline{146}$ \\
\hline Brazil & $\begin{array}{l}\text { Count } \\
\text { Exp Val } \\
\text { Row Pct } \\
\text { Col Pct } \\
\text { Adj Res }\end{array}$ & $\begin{array}{l}18 \\
16.8 \\
15.3 \% \\
21.4 \% \\
.3\end{array}$ & $\begin{array}{l}50 \\
42.1 \\
42.4 \% \\
23.8 \% \\
1.7\end{array}$ & $\begin{array}{l}12 \\
12.8 \\
10.2 \% \\
18.8 \% \\
-.3\end{array}$ & $\begin{array}{l}38 \\
46.3 \\
32.2 \% \\
16.5 \% \\
-1.7\end{array}$ & 118 \\
\hline Costa Rica & $\begin{array}{l}\text { Count } \\
\text { Exp Val } \\
\text { Row Pct } \\
\text { Col Pct } \\
\text { Adj Res }\end{array}$ & $\begin{array}{l}17 \\
10.4 \\
23.3 \% \\
20.2 \% \\
2.4\end{array}$ & $\begin{array}{l}16 \\
26.0 \\
21.9 \% \\
7.6 \% \\
-2.6\end{array}$ & $\begin{array}{l}4 \\
7.9 \\
5.5 \% \\
6.3 \% \\
-1.6\end{array}$ & $\begin{array}{l}36 \\
28.6 \\
49.3 \% \\
15.6 \% \\
1.9\end{array}$ & $\overline{73}$ \\
\hline China & $\begin{array}{l}\text { Count } \\
\text { Exp Val } \\
\text { Row Pct } \\
\text { Col Pct } \\
\text { Adj Res }\end{array}$ & $\begin{array}{l}18 \\
8.1 \\
31.6 \% \\
21.4 \% \\
3.9\end{array}$ & $\begin{array}{l}13 \\
20.3 \\
22.8 \% \\
6.2 \% \\
-2.1\end{array}$ & $\begin{array}{l}18 \\
6.2 \\
31.6 \% \\
28.1 \% \\
5.3\end{array}$ & $\begin{array}{l}8 \\
22.4 \\
14.0 \% \\
3.5 \% \\
-4.1\end{array}$ & 57 \\
\hline Sweden & $\begin{array}{l}\text { Count } \\
\text { Exp Val } \\
\text { Row Pct } \\
\text { Col Pct } \\
\text { Adj Res }\end{array}$ & $\begin{array}{l}20 \\
7.4 \\
38.5 \% \\
23.8 \% \\
5.2\end{array}$ & $\begin{array}{l}13 \\
18.5 \\
25.0 \% \\
6.2 \% \\
-1.7\end{array}$ & $\begin{array}{l}1 \\
5.7 \\
1.9 \% \\
1.6 \% \\
-2.2\end{array}$ & $\begin{array}{l}18 \\
20.4 \\
34.6 \% \\
7.8 \% \\
-.7\end{array}$ & 52 \\
\hline
\end{tabular}

Total Count

Note: $x^{2}(15, N=589)=123.40, p<.001$.

aDiffused ' $\quad$ boreclosed Achieved.

expected value fRow percentage ${ }^{\text {h}}$ Column percentage 'Adjusted standardized residuals 
Table 16

World View Status Distribution. Expected Value, and Adjusted Standardized Residuals by Site.

\begin{tabular}{|c|c|c|c|c|c|c|}
\hline Site & & Diffa $^{a}$ & Fore $^{b}$ & Mora $^{c}$ & $\mathrm{Achi}^{\mathrm{d}}$ & Total \\
\hline Miami & $\begin{array}{l}\text { Count } \\
\text { Exp Val } \\
\text { Row Pct } \\
\text { Col Pct } \\
\text { Adj Res } \\
\text { Ad }\end{array}$ & $\begin{array}{l}10 \\
20.7 \\
8.0 \% \\
10.9 \% \\
-2.9\end{array}$ & $\begin{array}{l}44 \\
43.4 \\
35.2 \% \\
22.8 \% \\
.1\end{array}$ & $\begin{array}{l}13 \\
19.6 \\
10.4 \% \\
14.9 \% \\
-1.8\end{array}$ & $\begin{array}{l}58 \\
41.4 \\
46.4 \% \\
31.5 \% \\
3.6\end{array}$ & $\overline{125}$ \\
\hline Nebraska & $\begin{array}{l}\text { Count } \\
\text { Exp Val } \\
\text { Row Pct } \\
\text { Col Pct } \\
\text { Adj Res }\end{array}$ & $\begin{array}{l}14 \\
22.5 \\
10.3 \% \\
15.2 \% \\
-2.3\end{array}$ & $\begin{array}{l}53 \\
47.2 \\
39.0 \% \\
27.5 \% \\
1.2\end{array}$ & $\begin{array}{l}25 \\
21.3 \\
18.4 \% \\
28.7 \% \\
1.0\end{array}$ & $\begin{array}{l}44 \\
45.0 \\
32.4 \% \\
23.9 \% \\
-.2\end{array}$ & $\overline{136}$ \\
\hline Brazil & $\begin{array}{l}\text { Count } \\
\text { Exp Val } \\
\text { Row Pct } \\
\text { Col Pct } \\
\text { Adj Res }\end{array}$ & $\begin{array}{l}16 \\
20.4 \\
13.0 \% \\
17.4 \% \\
-1.2\end{array}$ & $\begin{array}{l}47 \\
42.7 \\
38.2 \% \\
24.4 \% \\
.9\end{array}$ & $\begin{array}{l}13 \\
19.2 \\
10.6 \% \\
14.9 \% \\
-1.8\end{array}$ & $\begin{array}{l}47 \\
40.7 \\
38.2 \% \\
25.5 \% \\
1.4\end{array}$ & $\overline{123}$ \\
\hline Costa Rica & $\begin{array}{l}\text { Count } \\
\text { Exp Val } \\
\text { Row Pct } \\
\text { Col Pct } \\
\text { Adj Res }\end{array}$ & $\begin{array}{l}16 \\
10.9 \\
24.2 \% \\
17.4 \% \\
1.8\end{array}$ & $\begin{array}{l}18 \\
22.9 \\
27.3 \% \\
9.3 \% \\
-1.4\end{array}$ & $\begin{array}{l}8 \\
10.3 \\
12.1 \% \\
9.2 \% \\
-.8\end{array}$ & $\begin{array}{l}24 \\
21.8 \\
36.4 \% \\
13.0 \% \\
.6\end{array}$ & 66 \\
\hline$\overline{C h i n a}$ & $\begin{array}{l}\text { Count } \\
\text { Exp Val } \\
\text { Row Pct } \\
\text { Col Pct } \\
\text { Adj Res }\end{array}$ & $\begin{array}{l}27 \\
9.6 \\
46.6 \% \\
29.3 \% \\
6.5\end{array}$ & $\begin{array}{l}7 \\
20.1 \\
12.1 \% \\
3.6 \% \\
-3.8\end{array}$ & $\begin{array}{l}21 \\
9.1 \\
36.2 \% \\
24.1 \% \\
4.6\end{array}$ & $\begin{array}{l}3 \\
19.2 \\
5.2 \% \\
1.6 \% \\
-4.8\end{array}$ & 58 \\
\hline Sweden & $\begin{array}{l}\text { Count } \\
\text { Exp Val } \\
\text { Row Pct } \\
\text { Col Pct } \\
\text { Adj Res }\end{array}$ & $\begin{array}{l}9 \\
7.9 \\
18.8 \% \\
9.8 \% \\
.4\end{array}$ & $\begin{array}{l}24 \\
16.7 \\
50.0 \% \\
12.4 \% \\
2.3\end{array}$ & $\begin{array}{l}7 \\
7.5 \\
14.6 \% \\
8.0 \% \\
-2\end{array}$ & $\begin{array}{l}8 \\
15.9 \\
16.7 \% \\
4.3 \% \\
-2.5\end{array}$ & 48 \\
\hline
\end{tabular}

Total Count

Note. $X^{2}(15, N=556)=104.31, \mathrm{p}<.001$.

aDiffused ${ }^{b}$ Foreclosed ${ }^{\mathrm{d}}$ Achieved.

${ }^{e}$ Expected value 'Row percentage ${ }^{\text {h}}$ Column percentage 'Adjusted standardized residuals. 
Table 17

Mean of Satisfaction/Distress Ratings for the Three Identity Domains and

Standard Deviation (in Parentheses)

\begin{tabular}{lllll} 
& $\begin{array}{l}\text { Personal } \\
\text { development } \\
\text { satisfaction }\end{array}$ & $\begin{array}{l}\text { Interpersonal } \\
\text { development } \\
\text { satisfaction }\end{array}$ & $\begin{array}{l}\text { World } \\
\text { view } \\
\text { satisfaction }\end{array}$ & n of cases \\
\hline Miami & $7.17(1.37)$ & $7.59(1.16)$ & $7.30(1.03)$ & 157 \\
Nebraska & $6.96(1.29)$ & $7.60(1.09)$ & $7.21(.99)$ & 174 \\
Brazil & $7.32(1.17)$ & $8.11(1.12)$ & $7.34(.88)$ & 156 \\
Costa Rica & $7.57(1.12)$ & $7.42(.93)$ & $6.94(1.01)$ & 106 \\
China & $6.50(.92)$ & $6.55(.84)$ & $6.35(.80)$ & 88 \\
Sweden & $6.63(.92)$ & $7.17(.92)$ & $6.47(.58)$ & 74
\end{tabular}

Total N 
Table 18

Discriminant Analyses Predicting Identity Statuses in the World View Domain in Nebraska

Diffused Foreclosed Moratorium Achieved

\begin{tabular}{|c|c|c|}
\hline n $\%$ & $\begin{array}{l}\mathrm{n} \\
\%\end{array}$ & \\
\hline
\end{tabular}

\begin{tabular}{llllllllll}
\hline Diffused & 0 & 0 & 5 & 36 & 0 & 0 & 9 & 64 & 14
\end{tabular}

$\begin{array}{llllllllll}\text { Foreclosed } & 0 & 0 & 45 & 85 & 0 & 0 & 8 & 15 & 53\end{array}$

$\begin{array}{llllllllll}\text { Moratorium } & 0 & 0 & 14 & 56 & 0 & 0 & 11 & 44 & 25\end{array}$

$\begin{array}{llllllllll}\text { Achieved } & 0 & 0 & 23 & 52 & 0 & 0 & 21 & 48 & 44\end{array}$

$\begin{array}{llllll}N & 0 & 87 & 0 & 49 & 136\end{array}$

Note. $X^{2}(3, N=133)=12.53, p<.01$.

Within Groups Centroids

Function 1

Group Centroids

1. Diffused $\quad-.282$

2 .Forclosed $\quad .391$

3. Moratorium $\quad-.288$

4. Achieved $\quad-.221$

Note. Unstandardized canonical discriminant functions evaluated at group means. 
Table 19

Discriminant Analyses Predicting Identity Statuses in the World View

Domain in Brazil

Diffused Foreclosed Moratorium Achieved

$\overline{\mathrm{n} \%} \overline{\mathrm{n} \%} \overline{\mathrm{n} \%} \mathrm{~N}$

\begin{tabular}{llllllllll}
\hline Diffused & 4 & 25 & 7 & 44 & 0 & 0 & 5 & 31 & 16
\end{tabular}

$\begin{array}{llllllllll}\text { Foreclosed } & 4 & 9 & 16 & 34 & 0 & 0 & 27 & 57 & 47\end{array}$

$\begin{array}{llllllllll}\text { Moratorium } & 4 & 31 & 2 & 15 & 0 & 0 & 7 & 54 & 13\end{array}$

$\begin{array}{llllllllll}\text { Achieved } & 2 & 4 & 9 & 19 & 1 & 2 & 35 & 75 & 47\end{array}$

$\begin{array}{llllll}N & 14 & 34 & 1 & 74 & 123\end{array}$

Note. $X^{2}(6, N=114)=26.11, p<.001$.

Within Groups Centroids

Function

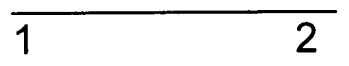

1. Diffused $\quad .822 \quad-.271$

2 .Forclosed $\quad-.159 \quad-.232$

3. Moratorium $\quad .522 \quad .624$

4. Achieved $\quad-.289 \quad .149$

Note. Unstandardized canonical discriminant functions evaluated at group means. 
Table 20

Within groups correlations and Discriminant Function Coefficients Predicting

Identity Statuses in the World View Domain in Brazil

Within Groups Correlations

Function

\begin{tabular}{lc}
\hline 1 & 2 \\
.783 & -.623 \\
.651 & .759
\end{tabular}

Standardized Canonical Discriminant Function Coefficients

Function

1

Gender

$.760 \quad-.652$

Institutional Change

$.623 \quad .783$


Table 21

Discriminant Analyses Predicting Identity Statuses in the World View Domain in China

\begin{tabular}{|c|c|c|c|c|c|c|c|c|}
\hline \multicolumn{2}{|c|}{ Diffused } & \multicolumn{2}{|c|}{ Foreclosed } & \multicolumn{2}{|c|}{ Moratorium } & \multicolumn{2}{|c|}{ Achieved } & \\
\hline$n$ & $\%$ & $\mathrm{n}$ & $\%$ & $\mathrm{n}$ & $\%$ & $\mathrm{n}$ & $\%$ & $\mathrm{~N}$ \\
\hline 23 & 85 & 1 & 4 & 3 & 11 & 0 & 0 & 27 \\
\hline
\end{tabular}

$\begin{array}{llllllllll}\text { Foreclosed } & 4 & 57 & 3 & 43 & 0 & 0 & 0 & 0 & 7\end{array}$

$\begin{array}{llllllllll}\text { Moratorium } & 19 & 91 & 1 & 5 & 1 & 5 & 0 & 0 & 21\end{array}$

$\begin{array}{llllllllll}\text { Achieved } & 1 & 33 & 0 & 0 & 2 & 67 & 0 & 0 & 3\end{array}$

$\begin{array}{llllll}N & 47 & 5 & 6 & 0 & 58\end{array}$

Note. $X^{2}(3, N=55)=10.63, \underline{p}<.01$.

Within Groups Centroids and Correlations

Function 1
1. Diffused
$-.387$
2. Forclosed
.843
3. Moratorium
.100
4. Achieved
.912

Note: Unstandardized canonical discriminant functions evaluated at group means. 


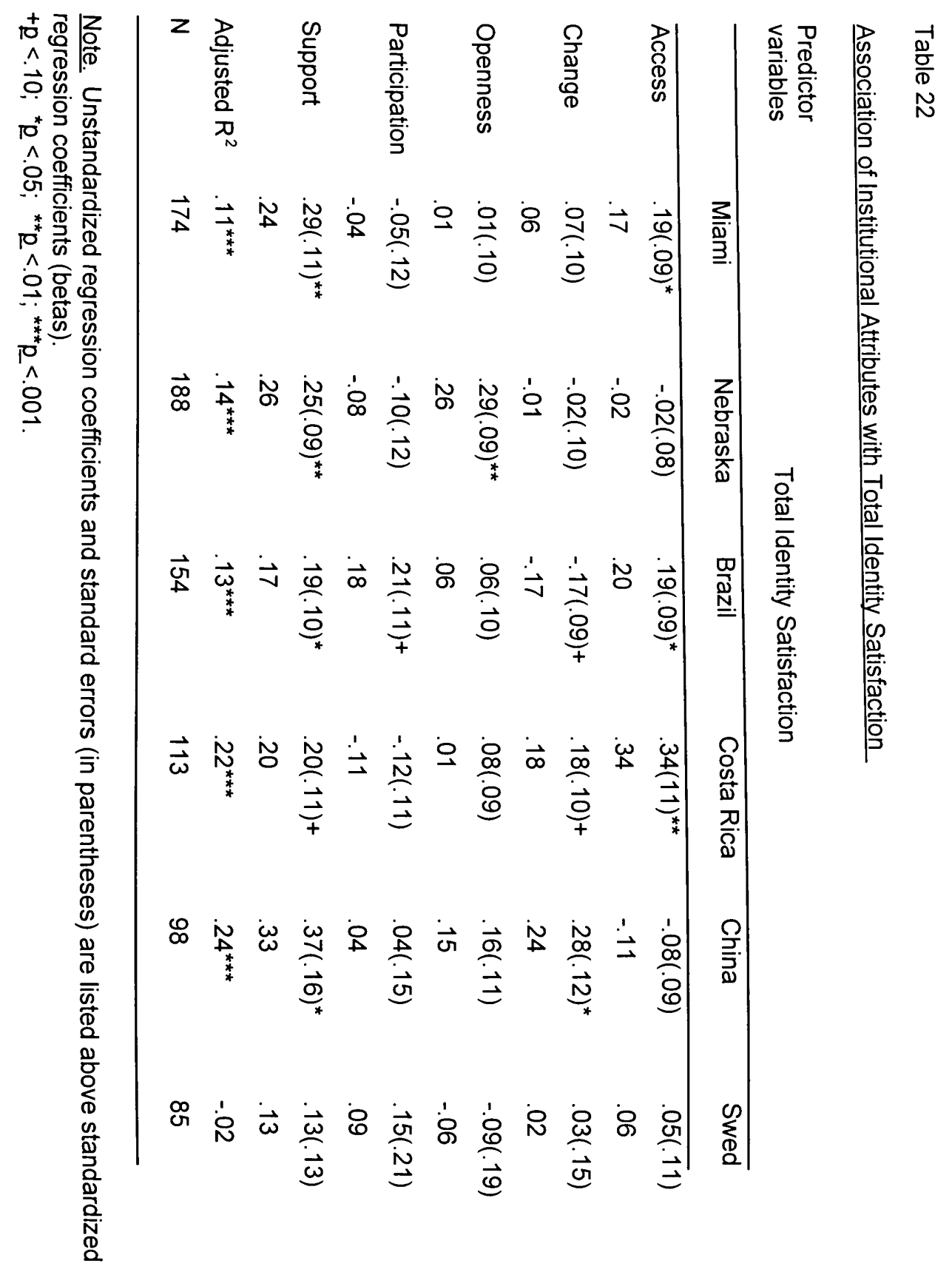




\section{VITA}

\section{ERVIN BRIONES}

September 7, 1963

1992

1992-1993

1995

1994-1997
Born, Esteli, Nicaragua

B.A., Psychology

Graduated with Honors

Florida International University

Miami, Florida

Mental Health Technician

North Shore Hospital

North Miami Beachi, Florida

M.S., Psychology

Florida International University

Miami, Florida

Research Associate

Center for Family Studies

Department of Psychiatry

School of Medicine

University of Miami

\section{PUBLICATIONS AND PRESENTATIONS}

Berman, S., Wanderman, J., Briones, E., Wolf, L., \& Kurtines, W. (1993). Education for democracy: A co-constructivist perspective. Theory of Education. $\underline{5}, 87-96$.

Briones, E., Berman, S., Schmaltz, J., Ferrer, L., \& Kurtines, W. (1996, March). Social institutions and identity formation: The interface between society and the individual. Poster session presented at the Society for Research on Adolescence, Boston, MA.

Briones, E. (1997, March). The Influence of culture and institutional change on identity formation among late adolescents from Brazil, Costa Rica, and the USA. Paper presented at the Society for Research on Identity Formation, Miami Beach, Florida. 